\title{
MANUFACTURING AND INSTRUMENTATION OF AN OPEN END COMPRESSED AIR SHOCK TUBE
}

\author{
A Thesis \\ presented to \\ the Faculty of California Polytechnic State University, \\ San Luis Obispo
}

\author{
In Partial Fulfillment \\ of the Requirements for the Degree \\ Master of Science in Aerospace Engineering
}

by

Josue O. Ruiz

December 2017 
(C) 2017

Josue O. Ruiz

ALL RIGHTS RESERVED 
TITLE: $\quad$ Manufacturing and Instrumentation of an Open End Compressed Air Shock Tube

AUTHOR: Josue O. Ruiz

DATE SUBMITTED: $\quad$ December 2017

COMMITTEE CHAIR: Graham Doig, Ph.D. Assistant Professor

Aerospace Engineering Department

COMMiTTEE MEMBER: Patrick Lemieux, Ph.D.

Professor

Mechanical Engineering Department

COMMitTEE MEMBER: Aaron Drake, Ph.D.

Associate Professor

Aerospace Engineering Department

COMMitTEE MEMBER: David D. Marshall, Ph.D.

Department Chair

Aerospace Engineering Department 


\begin{abstract}
Manufacturing and Instrumentation of an Open End Compressed Air Shock Tube
\end{abstract}

Josue O. Ruiz

Shock tubes have been used to study shock wave structures and high speed flow features. The purpose of constructing this open end shock tube was to have the ability to produce shock waves in a laboratory setting but also understand the exit flow coming out which can be applied to future studies that are beyond the scope of this work. This undertaking would require that an open end shock tube be built and instrumented with PCB Integrated Circuit Piezoelectric (ICP) Pressure Sensor Model 113B24 that would then be connected to a PCB Model 482C05 Signal Conditioner to measure the pressure jumps as well as the speed of the shock wave. The data was acquired using National Instruments NI PXIe-1071 chassis with a PXI 1088 Embedded Controller as well as three PXI 5114 digitizer cards with the Virtual Instrument coded using LabView. The data was written to a text file that was then transferred to MATLAB for post processing using a Savitzy-Golay filter to clean up the signal noise. The shock tube was driven using compressed air and a diaphragm burst was achieved through spontaneous rupture of a 0.003" Mylar diaphragm. The open shock tube built for this undertaking fits in a lab space and successfully produces a shock wave that propagates down the tube that exits at the open end to reproduce a blast wave. Additionally the available pressure sensors and DAQ were integrated into the shock tube to measure the different predicted shock structures in each run. The test data at the exit of the shock tube demonstrate the expected exit flow features, but a flow visualization is necessary to get a better understanding of the exit flow. 


\section{ACKNOWLEDGMENTS}

I would like to first off give a big thank you to Dr. Graham Doig for all the help you provided during the completion of this thesis. His knowledge and support

were no doubt invaluable to me and I wouldn't have made to the finish line without him.

Dr. Patrick Lemieux, your knowledge of shock tubes and attention to details helped in laying out a clear and concise plan to develop a working shock tube.

There's also Cody Thompson thank you for making all parts needed for and providing practical knowledge that I wouldn't have had anywhere else except from someone with years of experience in manufacturing.

I would also like to give thanks to my friend Tony Tan and other multiple students that helped conduct the multiple runs with me to get all my data.

Alex Crawford thank you for reading my thesis and taking the time to proofread it, but also for believing in me and supporting me until the end.

Lastly I would like to thank my parents who always told me to never stop chasing my dreams and were always there to help me the best they could. 


\section{TABLE OF CONTENTS}

LIST OF TABLES . . . . . . . . . . . . . . . . . . ix

LIST OF FIGURES . . . . . . . . . . . . . . . . x

NOMENCLATURE .................... xiii

1 Introduction . . . . . . . . . . . . . . . . . 1

1.1 Open End Shock Tubes . . . . . . . . . . . . . . . . 2

1.2 Boundary Layer in the Shock Tube . . . . . . . . . . . . . . . . . 11

1.3 Blast Waves . . . . . . . . . . . . . . . . . . . . . 13

1.4 Exit Flow of an Open End Shock Tube . . . . . . . . . . . . 16

2 Design and Manufacturing of Open End Shock Tube . . . . . . . . . 18

2.1 Design Requirements of the Shock Tube . . . . . . . . . . . . . 18

2.2 Selecting the Driver Gas of the Shock Tube . . . . . . . . . . . . . 19

2.3 Selecting the General Dimension of the Shock Tube . . . . . . . . 20

2.4 Determining the Material and Thickness of Shock Tube Walls . . 21

2.5 Sealing the Shock Tube . . . . . . . . . . . . . . . 23

2.6 Diaphragm Selection . . . . . . . . . . . . . 26

2.7 Selection and Installation of Air Valves and Pressure Gauge . . . 28

2.8 Making of Sensor Fixtures and Installation . . . . . . . . . . . . . 31 
2.9 Test Stand and Installing Shock Tube . . . . . . . . . . . . . . . . 33

3 Apparatus and Set-Up . . . . . . . . . . . . . . . . . 35

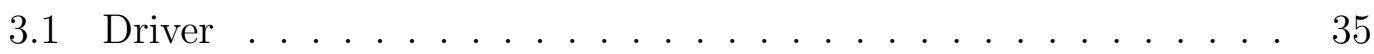

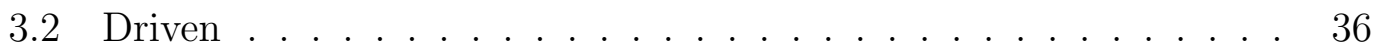

3.3 Diaphragm and Flange . . . . . . . . . . . . . . . . . . . 36

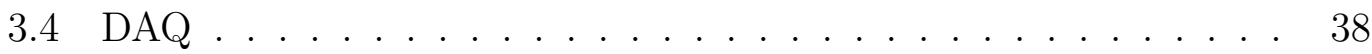

4 Experimental Methodology and Procedure . . . . . . . . . . . . . 42

4.1 Pressurizing the Driver Tube . . . . . . . . . . . . . . . 42

4.2 Sensors . . . . . . . . . . . . . . . . . . . . . 42

4.3 DAQ Set Up . . . . . . . . . . . . . . . . . 44

4.4 Characterization of the Shock Tube . . . . . . . . . . . . . . 44

5 Results and Conclusion . . . . . . . . . . . . . . . . 47

5.1 Diaphragm Rupture . . . . . . . . . . . . . . 47

5.2 Shock Velocity. . . . . . . . . . . . . . . . . . 48

5.3 Incident (Parallel) Pressure _ . . . . . . . . . . . . . . . . . . . . 49

5.4 Total / Reflected Pressure Measurements . . . . . . . . . . . . . 52

5.5 Blast Wave Profiling . . . . . . . . . . . . . . . . 58

5.6 Conclusions .......................... 61

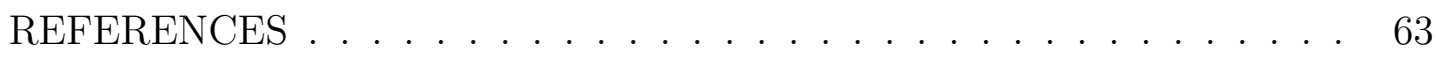

\section{APPENDICES}

A Sample Calculations .................... 66

A.1 Shock Tube Thickness Calculation . . . . . . . . . . . . 67

A.2 Number of Bolts . . . . . . . . . . . . . . . . . 68 
A.3 Selecting Voltage Range . . . . . . . . . . . . . . . . . . 70

A.4 Determining Thickness of Mylar Diaphragm . . . . . . . . . . . 72

B PCB Adapter Drawing . . . . . . . . . . . . . . 73

C Testing Documents ..................... 75

C.1 Safe Working Procedure . . . . . . . . . . . . . . 76

C.2 Test Procedure . . . . . . . . . . . . . . . . . . . . 82

C.3 MATLAB Code for Post Processing . . . . . . . . . . . . . . 88

D Unfiltered Data ........................ 101 


\section{LIST OF TABLES}

Table Page

2.1 Shock Tube Dimensions . . . . . . . . . . . . . . . . . . 20

4.1 Settings for the DAQ Cards . . . . . . . . . . . . . 44

5.1 Shock Mach Number . . . . . . . . . . . . . . . . . . . . . . . 49

5.2 Reflected/Total Pressure Summary . . . . . . . . . . . . 58 


\section{LIST OF FIGURES}

Figure Page

1.1 Open End Shock Tube Before Diaphragm Rupture . . . . . . . . 3

1.2 Open End Shock Tube After Diaphragm Rupture . . . . . . . . . 3

1.3 Flow Velocities After Diaphragm Rupture . . . . . . . . . . 5

1.4 Moving vs. Fixed Frame of Reference for Shock . . . . . . . . . 6

1.5 Shock Tube Pressure Ratio vs Shock Strength and Mach Shock Number ...................... . . 10

1.6 Wave Propagation in the Open End Shock Tube . . . . . . . . . . 10

1.7 Boundary Layer following Initial Shock $[1]$. . . . . . . . . . . . . . 11

1.8 Boundary Layer in Moving Coordinate System[1] . . . . . . . . . 11

1.9 Time History for Properties of a Blast Wave[2] . . . . . . . . . . . 13

1.10 Pressure Sensor Positions Relative to the Direction of Travel of the Blast Wave . . . . . . . . . . . . . . . . . . . . 14

1.11 Theoretical Friedlander Wave . . . . . . . . . . . . . . . . . . . 16

1.12 Vortex Rings at Exit of Open End Shock Tube[3] . . . . . . . . . 17

1.13 Exit Flow Later in Time[4] . . . . . . . . . . . . . . . . 17

2.1 Hoop and Longitudinal Stresses in Thin Wall Vessel[5] . . . . . . 22

2.2 Force Diagram for Determining Number of Bolts . . . . . . . . . . 24

2.3 Flange Connection with Gasket and Diaphragm . . . . . . . . . . 26

2.4 Appearance of Flow following Diaphragm Rupture[6] . . . . . . . 27 
2.5 NPT Look Up Table Reference Figure[7] . . . . . . . . . . . . 29

2.6 Schrader Valves Installed on Shock Tube . . . . . . . . . . . 30

2.7 Bourdon Pressure Gauge Installed on Shock Tube . . . . . . . . 31

2.8 Piezoelectric Pressure Sensor Installed on Shock Tube . . . . . . . 32

2.9 Piezoelectric Pressure Sensor at Exit of the Shock Tube . . . . . . 32

2.10 Test Stand Arm Interference . . . . . . . . . . . . . . . . . 33

2.11 Hose Clamp with Rubber Sleeve on Shock Tube . . . . . . . . . 34

2.12 Shock Tube Installed on Test Stand . . . . . . . . . . . . . . 34

3.1 Experimental Set Up of Shock Tube . . . . . . . . . . . . . . 35

3.2 Assembly of Flange with Diaphragm and Gasket . . . . . . . . . 37

3.3 Tigthening Pattern of Flange Bolts _ . . . . . . . . . . 38

3.4 Front Page of LabView VI . . . . . . . . . . . . . . . . . . . 39

3.5 Producer/Consumer Design Pattern[8] . . . . . . . . . . . 40

3.6 For Loop to set Correct Time . . . . . . . . . . . . . . . . . 41

4.1 Schrader Valve Air Hose Connection _. . . . . . . . . . . . 43

4.2 Signal Conditioner, Chassis, and DAQ Cards . . . . . . . . . . 43

4.3 Sensor Configurations at Exit $\ldots \ldots \ldots \ldots$

5.1 Typical Pressure Data of P4 Sensor . . . . . . . . . . . . . . 48

5.2 Typical Data of Incident Pressure Sensors _ . . . . . . . . . . 49

5.3 Estimated Position of Sensor Inside the Tube . . . . . . . . 51

5.4 Typical Data of Reflected/Total Pressure Sensors . . . . . . . . 52

5.5 Pencil Mount for Total Pressure Gauge . . . . . . . . . . . . . . 54

5.6 Pressure Data for Sensors in Position AII . . . . . . . . . . . 55

5.7 Pressure Data for Sensors in Position BII . . . . . . . . . . 56

5.8 Additional Flow Features in Position BII . . . . . . . . . . 57 
5.9 Comparison to Friedlander Wave Position BI . . . . . . . . . . . . 58

5.10 Comparison to Friedlander Wave Position AI . . . . . . . . . . . . 59

5.11 Comparison to Friedlander Wave to Incident Pressure . . . . . . . 60

5.12 Time History of Incident Pressure Measurements . . . . . . . . . 61

D.1 Unfiltered Incident Pressure Measurements . . . . . . . . . . . . . 102

D.2 Unfiltered AI Pressure Measurements . . . . . . . . . . . . . 103

D.3 Unfiltered AII Pressure Measurements . . . . . . . . . . . . . . . 104

D.4 Unfiltered BI Pressure Measurements . . . . . . . . . . . . . 105

D.5 Unfiltered BII Pressure Measurements . . . . . . . . . . . . . 106

D.6 Unfiltered Velocity/Pressure Measurements . . . . . . . . . . . 107 


\section{NOMENCLATURE}

$\frac{p_{2}}{p_{1}} \quad$ Shock Strength

$\frac{p_{4}}{p_{1}} \quad$ Shock Tube Pressure Ratio

$\gamma_{1} \quad$ Specific Heat Ratio in Region 1 of Shock Tube Events

$\gamma_{2} \quad$ Specific Heat Ratio in Region 2 of Shock Tube Events

$\gamma_{3} \quad$ Specific Heat Ratio in Region 3 of Shock Tube Events

$\gamma_{4} \quad$ Specific Heat Ratio in Region 4 of Shock Tube Events

$\sigma_{H} \quad$ Hoop Stress

$\sigma_{L} \quad$ Longitudinal Stress

$\sigma_{\text {ult }} \quad$ Ultimate Stress

$\tau \quad$ Time

$a_{1} \quad$ Speed of Sound in Region 1 of Shock Tube Events

$a_{2} \quad$ Speed of Sound in Region 2 of Shock Tube Events

$a_{3} \quad$ Speed of Sound in Region 3 of Shock Tube Events

$a_{4} \quad$ Speed of Sound in Region 4 of Shock Tube Events

c Coefficient of Friction 
$M_{D} \quad$ Major Diameter of Bolt

$M_{s} \quad$ Shock Mach Number

$n \quad$ Number of Bolts

$N_{\text {bolt }}$ Preload Force in Bolt

$N_{\text {flange }}$ Distributed Force in the Flange

op Overpressure

$o p_{R} \quad$ Reflected Overpressure

$o p_{t o t}$ Total Overpressure

p Pressure

$p_{1} \quad$ Pressure in Region 1 of Shock Tube Events

$p_{2} \quad$ Pressure in Region 2 of Shock Tube Events

$P_{3} \quad$ Riemann Invariant in Region 3 of Shock Tube Events

$p_{3} \quad$ Pressure in Region 3 of Shock Tube Events

$P_{4} \quad$ Riemman Invariant in Region 4 of Shock Tube Events

$p_{4} \quad$ Pressure in Region 4 of Shock Tube Events

$p_{I} \quad$ Incident Pressure

$p_{R} \quad$ Reflected Pressure

$p_{a m b} \quad$ Ambient Pressure

psia Pounds per Square Inch Absolute 
psig Pounds per Square Inch Gauge

T Torque applied to Bolts

$t \quad$ Thickness

$t^{+} \quad$ Duration of Positive Phase

$u_{1} \quad$ Flow Velocity in Region 1 of Shock Tube Events

$u_{2} \quad$ Flow Velocity in Region 2 of Shock Tube Events

$u_{3} \quad$ Flow Velocity in Region 3 of Shock Tube Events

$u_{4} \quad$ Flow Velocity in Region 4 of Shock Tube Events

$u_{s} \quad$ Shock Velocity

$u_{c s} \quad$ Velocity of Contact Surface

$v_{1} \quad$ Converted Flow Velocity in Region 1 of Shock Tube Events

$v_{2} \quad$ Converted Flow Velocity in Region 2 of Shock Tube Events 


\section{Chapter 1}

Introduction

Shock tubes have been used extensively by those conducting research with shock waves as they have proven to be a highly reliable yet simple method to study shock structures, wave propagation, blast waves[9], and work with combustion processes [3]. The goal of this thesis work was to design and manufacture a shock tube, then to instrument it using Integrated Circuit Piezoelectric (ICP) Pressure Sensors in their "as is" state with their respective signal conditioner to measure the pressure jumps with the sensors being parallel to and in the flow of the shock wave, while using a National Instrument Digitizer to serve as a Data Acquisition System (DAQ), and post process the data using MATLAB. Shock tubes can be adjusted to fit a particular research field and for this case an open end shock tube was desired in order to have a facility whose exit flow would allow blast or shock waves fields to be produced in a laboratory setting. Also it was decided that the best way to proceed was for the shock tube to be driven by compressed air as it wouldn't require any extra facilities to be developed to handle different type of gases that can be used to drive a shock tube which would lead to the most timely method to verify the capabilities of the ICP sensors and DAQ. Lastly a circular cross section was used for these type of tubes and its required connectors/parts are readily available compared to those with cross sections of other shapes. 


\subsection{Open End Shock Tubes}

Before beginning to specifically describe the details of an open end shock tube it is first necessary to go over some general concepts that apply to most shock tubes. A standard shock tube works on the principle of having a section of higher pressure gas separated from a section of lower pressure gas and then instantaneously releasing the higher pressure gas into the lower pressure gas, this action will lead to the sudden expansion of air at supersonic speed to form a discontinuity which is known as a shock wave. This is accomplished with 3 components: the driver tube, the driven tube, and a diaphragm. The driver tube is the section where the driver gas or high pressure gas is held, the driven section or the low pressure section is where the shock wave will travel after the high pressure gas is released, and the diaphragm is what keeps the two sections separate. The most common way to release the gas in the driver section into the driven section is by bursting the diaphragm. This can be accomplished by selecting a diaphragm made out of a material that will rupture when the desired pressure ratio in the driver section is reached or by choosing a diaphragm that can withstand the pressure in the driver section but is later ruptured using a sharp needle or other mechanism. In figure 1.1 the shock tube is shown prior to diaphragm bursting with the specific regions labeled accordingly. 


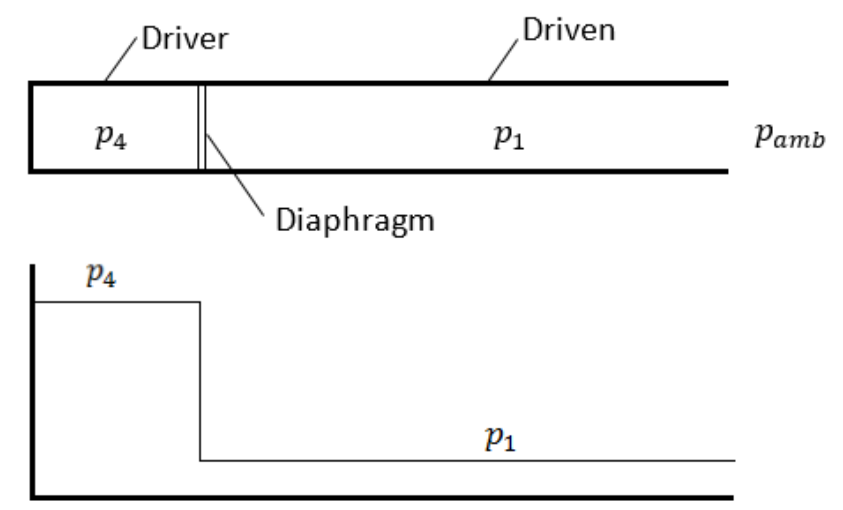

\section{Figure 1.1: Open End Shock Tube Before Diaphragm Rupture}

From figure $1.1 p_{4}$ is meant to represent the pressure in the driver section while $p_{1}$ is representing the pressure the driven section. It is important to note that what makes this figure relevant to open end shock tubes is that since the end of the tube is open $p_{1}$ is equal to ambient pressure represented by $p_{a m b}$. For any other type of shock the driven region can be closed off and be set to a different pressure. At the time when the diaphragm bursts we see the following events occurring inside the shock tube.

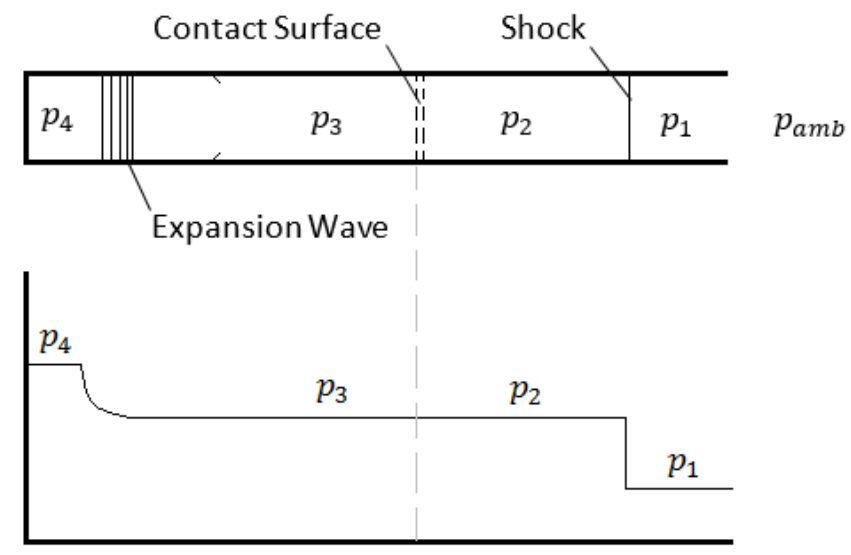

Figure 1.2: Open End Shock Tube After Diaphragm Rupture 
With the diaphragm ruptured an expansion wave is created, a contact surface and a shock begin to travel within the tube. The contact surface can be imagined to be acting as a piston that is driving the air in front of it which in turn produces the a shock wave that moves down the tube. The expansion wave travels upstream while the contact surface and the shock travel downstream. Each one of these regions can be related to one another by the pressure ratio between them. There two important pressure ratios that are used, the first is $\frac{p_{2}}{p_{1}}$ which is the pressure ratio of the shock caused by its pressure jump, this pressure ratio can also be considered the strength of the shock wave. The second is $\frac{p_{4}}{p_{1}}$ which is the pressure ratio between the driver and driven sections of the shock tube before the diaphragm bursts. With both these values along with compressible flow and shock wave theory the shock tube equation can be derived along with other relevant equations[9]. These are used to determine various shock structure values that will be used to validate the experimental values obtained from the shock tube. The following equations that are derived can be applied in general to any shock tube but it will be specified when a certain condition will only apply to an open end shock tube. The first condition established to derive the shock tube equation begins at the contact surface:

$$
p_{2}=p_{3}
$$


There is no pressure rise across the contact surface thus we can use equation 1.1 as one of the conditions that will be used to derive the necessary equations to predict the shock wave structures. The next set of conditions come from looking at the velocities insides the shock tube as shown in the figure 1.3 below.

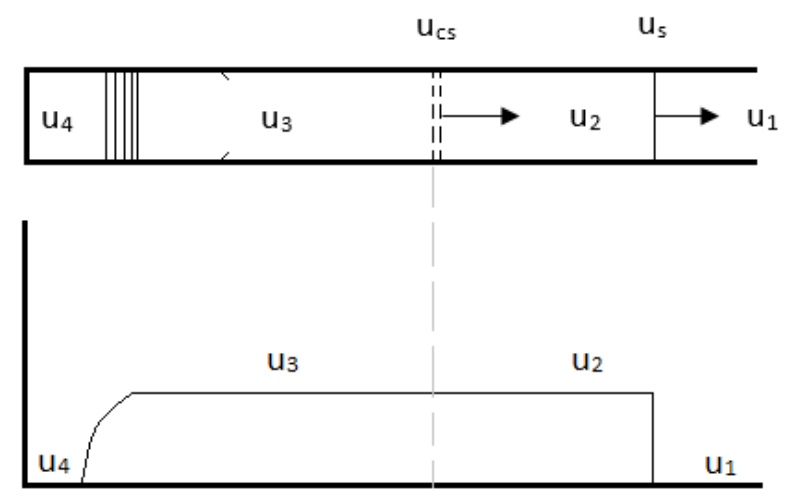

\section{Figure 1.3: Flow Velocities After Diaphragm Rupture}

From Figure 1.3 there are a total of 6 velocities that are relevant to understanding the shock structures inside the shock tube after burst. The two velocities that get added on as they do not correspond to a previously denoted section is velocities $u_{c s}$ which is the velocity at which the contact surface is moving and $u_{s}$ is the speed of the shock. These are labeled above their corresponding event in the shock tube as well as the arrows show the velocity direction. The following are the velocity conditions inside the shock tube.

$$
\begin{gathered}
u_{4}=u_{1}=0 \\
u_{2}=u_{3}=u_{c s}
\end{gathered}
$$


To obtain the shock jump relations it becomes necessary to change from a moving frame of reference (left) to a fixed frame of reference with respect to the shock (right) as shown in figure 1.4.
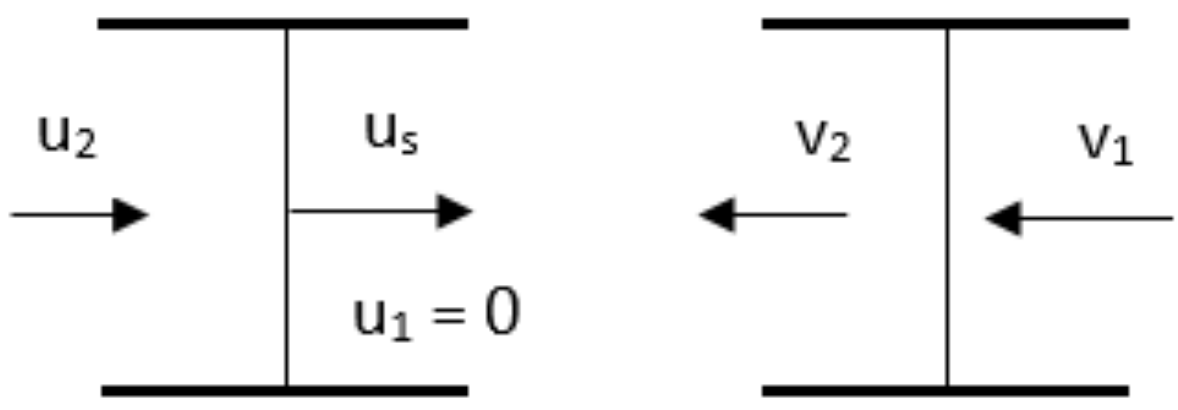

Figure 1.4: Moving vs. Fixed Frame of Reference for Shock

The following are flow velocity relationships between the fixed frame of reference and moving frame of reference with the shock.

$$
\begin{gathered}
v_{1}=-u_{s} \\
v_{2}=-u_{s}+u_{2}
\end{gathered}
$$

Using shock wave equations [9] the following relationship can be made between the flow velocities as shown and the Mach number of the shock wave as shown in equation 1.7. The Mach number of the shock wave is defined below.

$$
M_{s}=u_{s} / a_{1}
$$

Where $a=\sqrt{\frac{\gamma * p}{\rho}}$ or the speed of sound in the specified region. 


$$
\frac{v_{2}}{v_{1}}=\frac{u_{s}}{u_{s}-u_{2}}=\frac{\left(\gamma_{1}+1\right) M_{s}^{2}}{2+\left(\gamma_{1}-1\right) M_{s}^{2}}
$$

This equation can now be arranged to solve for $u_{2}$ and using equation 1.3 the velocity of the contact surface can be determined if the Mach number of the shock wave is known.

$$
u_{c s}=\frac{2 a_{1}}{\gamma_{1}+1}\left(M_{s}-\frac{1}{M_{s}}\right)
$$

To determine the Mach number of the shock wave the shock jump relations for pressure can be used as shown below:

$$
\frac{p_{2}}{p_{1}}=1+\frac{2 \gamma_{1}}{\gamma_{1}+1}\left(M_{s}-\frac{1}{M_{s}}\right)
$$

This can then be rearranged to the following:

$$
M_{s}^{2}=\frac{\gamma_{1}+1}{2 \gamma_{1}}\left(\frac{p_{2}}{p_{1}}\right)+\frac{\gamma_{1}-1}{\gamma_{1}+1}
$$

To proceed forward, to get the shock tube equation, the next step is to look at the relationship between section 3 and 4 . Sections 3 and 4 of the shock tube are separated by an expansion wave which happens to be an isentropic process allowing the use of isentropic relationships.

$$
\frac{p_{4}}{p_{3}}=\left(\frac{a_{4}}{a_{3}}\right)^{\frac{2 \gamma_{4}}{\gamma_{4}-1}}
$$

In the shock tube the expansion wave is considered to be an expansion fan or a series of waves originating from a common point in time and space as shown in figure 1.2. To develop the equations necessary to obtain the flow velocities the 
continuity equation for a nonsteady one-dimensional flow is used to derive the equation to describe the flow.

$$
\frac{1}{\rho} \frac{D \rho}{D \tau}+\frac{\partial u}{\partial \tau}=0
$$

To solve this differential equation the method of characteristics must be employed using Riemann invariants along the characteristics lines [9]. Along the characteristic lines (or the Mach lines when specifically dealing with shocks) the invariants can be said to be constant and for the case of our expansion fan in the shock tube it is considered to be a left facing wave so that the following can be said:

$$
u+\frac{2}{\gamma-1} a=P=\text { constant }
$$

Where $\mathrm{P}$ is considered to be the invariant (for a right facing wave the invariant is considered to be Q). Since the condition set forth holds true across the expansion fan in the shock tube then:

$$
P_{4}=P_{3}
$$

With the invariants of section 3 and 4 equal to one another and using equation 1.13

$$
u_{4}+\frac{2}{\gamma_{4}-1} a_{4}=u_{3}+\frac{2}{\gamma_{3}-1} a_{3}
$$

As established by equation 1.2 since the gas in section 4 is at rest equation 1.15 is then reduced to the following:

$$
\frac{2}{\gamma_{4}-1} a_{4}=u_{3}+\frac{2}{\gamma_{3}-1} a_{3}
$$


Before using our previously established equations to get to the shock tube equation there is an identity that must be considered as well, it is:

$$
\frac{p_{3}}{p_{4}}=\frac{p_{3}}{p_{2}} \frac{p_{2}}{p_{1}} \frac{p_{1}}{p_{4}}
$$

Pairing this identity with equation 1.9 and combining equations $1.3,1.8,1.11$ and 1.16 yields the shock tube equation.

$$
\frac{p_{4}}{p_{1}}=\left[1+\frac{2 \gamma_{1}}{\gamma_{1}+1}\left(M_{s}^{2}-1\right)\right]\left[\frac{1}{1-\frac{\gamma_{4}-1}{\gamma_{1}+1} \frac{a_{1}}{a_{4}}\left(M_{s}-\frac{1}{M_{s}}\right)}\right]^{\frac{2 \gamma_{4}}{\gamma_{4}-1}}
$$

Note that this is derived assuming $\gamma_{4}=\gamma_{3}$, likewise it is possible to just use the identity from equation 1.16 with equation 1.9 to get the driver and driven pressure ratio in terms of the shock strength.

$$
\frac{p_{4}}{p_{1}}=\frac{p_{2}}{p_{1}}\left(1-\frac{\left(\gamma_{4}-1\right)\left(\frac{a 1}{a 4}\right)\left(\frac{p_{2}}{p_{1}}-1\right)}{\sqrt{4 \gamma_{1}^{2}+2 \gamma_{1}\left(\gamma_{1}+1\right)\left(\frac{p_{2}}{p_{1}}\right)}}\right)^{-\left(\frac{2 \gamma_{4}}{\gamma_{4}-1}\right)}
$$

In figure 1.5 are the expected trends for equations 1.17 and 1.18 with the assumptions that $\gamma_{4}=\gamma_{1}=1.4, a_{1}=a_{4}$ and that both the driver and driven sections have the same gas inside of them. As a quick side by side comparison a pressure ratio $\frac{p_{2}}{p_{1}}=4.5$ corresponds to $M_{s}=2$ which requires a $\frac{p_{4}}{p_{1}} \approx 33.7$ 

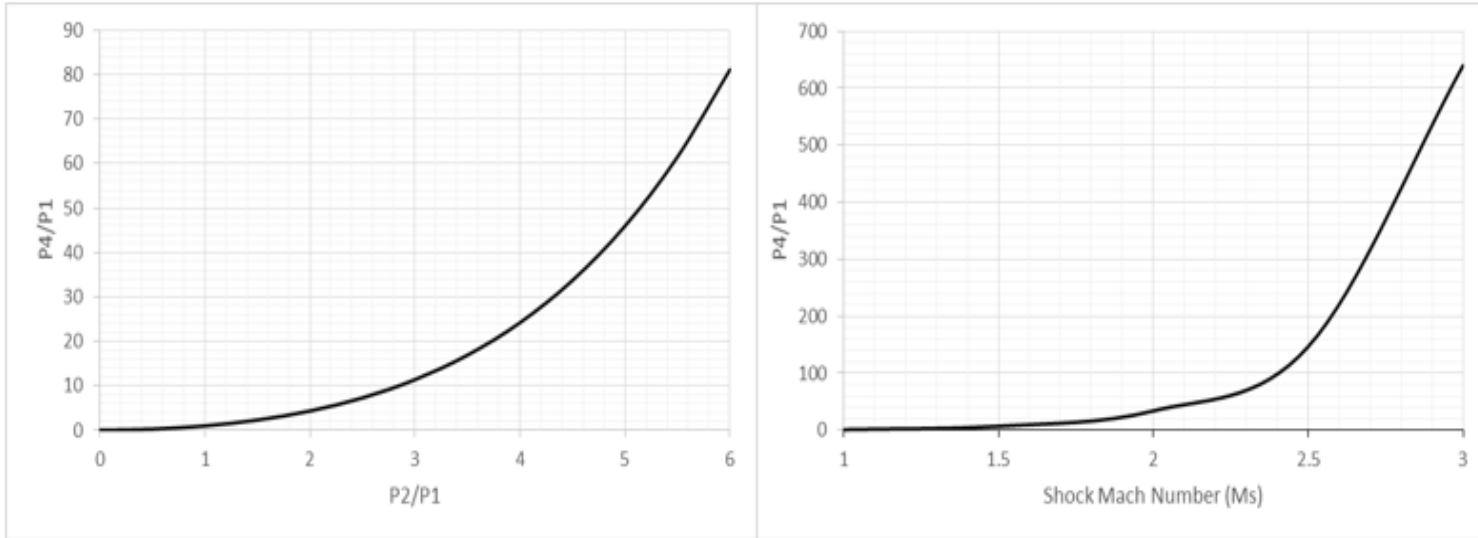

Figure 1.5: Shock Tube Pressure Ratio vs Shock Strength and Mach Shock Number

In figure 1.6 the shock wave propagation is shown in a $\mathrm{x}$-t diagram to illustrate the expected behavior of the shock in another fashion. The important thing to note is that in a general type of shock tube this diagram will differ since the other end is closed. Instead of the shock reflecting off the wall and interacting with the contact surface, the shock and the contact surface will propagate out of the open end of the tube and develop a different type of flow as it interacts with the ambient air.

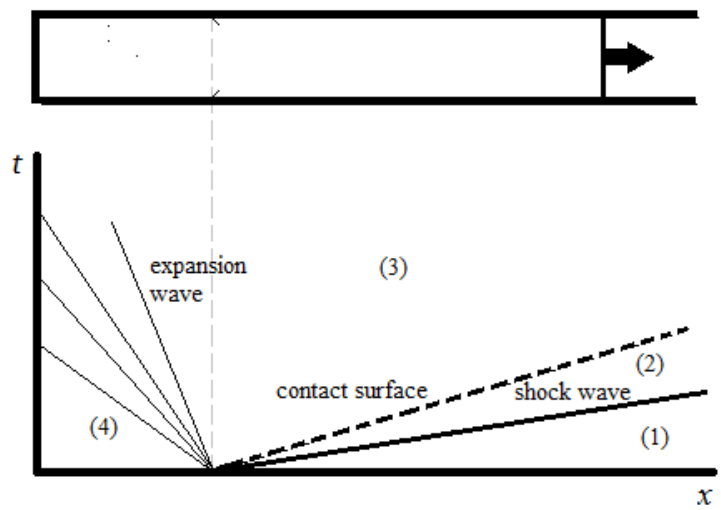

Figure 1.6: Wave Propagation in the Open End Shock Tube 


\subsection{Boundary Layer in the Shock Tube}

The flow that follows the shock wave is bounded by the walls of the shock tube and will lead to the formation of a boundary layer on the walls. This flow problem is described to be non-stationary, viscous, compressible and involves heat transfer [1]. The general set up of this problem is described in figure 1.7.

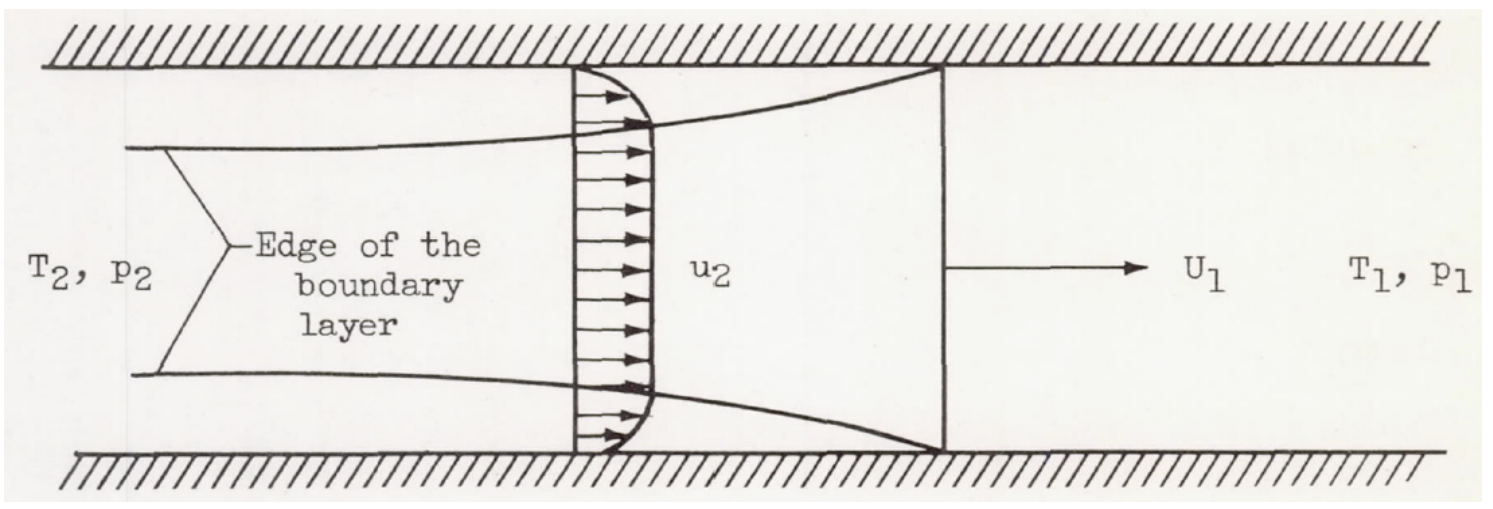

Figure 1.7: Boundary Layer following Initial Shock [1]

Transferring into a moving coordinate system with reference to the shock wave makes the problem steady. The setup looks like the classical Blassius problem of viscous flow over flat plate in a free stream [1].

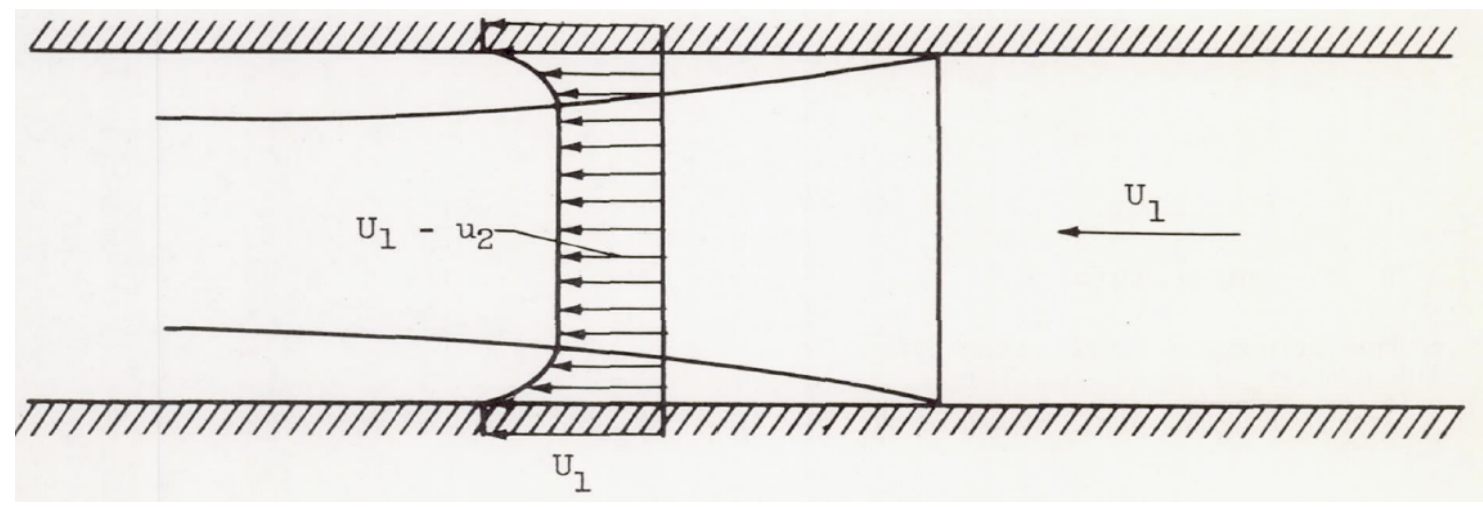

Figure 1.8: Boundary Layer in Moving Coordinate System [1] 
There three differences to this problem that deviate it from the Blassius problem. First, there is a pressure distribution in the tube, second, the flow is compressible, and third, the wall in the current coordinate system is not stationary. With these differences considered the motion equation is still the same as the Blassius problem $\left(f^{\prime \prime}+f^{\prime \prime \prime}=0\right)$ with the major difference being the boundary conditions where the velocity at the wall is not zero, in this case $f^{\prime}(0)=\frac{U_{1}}{U_{1}-u_{2}}$. The motion equation has been solved numerically [1] but it is not the intent of this thesis to delve into this, rather briefly describe the boundary layer that exists and how the effects of it lead to shock wave attenuation.

The biggest contributor to shock wave attenuation is energy lost from the test gas to the boundary layer [10]. This leads to the deceleration of the shock and acceleration of the contact surface which in turn results in non-constant values for the flow quantities. Although to fully understand how the boundary layer will affect the shock it needs to be combined with the effect of the non-instantaneous burst of the diaphragm. In the 'real' shock tube the shock wave is not created immediately but rather formed from a coalescence of compression waves being formed due to the progressive opening of the diaphragm. This combined with the boundary layer will cause the shock wave to have a period of acceleration, reach a maximum velocity and then begin to decelerate so generally a long driver length is required to achieve a constant wave speed [11]. How much of an effect these features described will have relies on the experimental conditions. 


\subsection{Blast Waves}

A blast wave is defined to be a type of shock wave which decays immediately after the peak is reached. The decay occurs in all the properties of the wave and the rate of decay is different for each parameter [12] but the decay will occur in an exponential fashion [2]. It is important to note as well that this type of wave is typically unbounded.

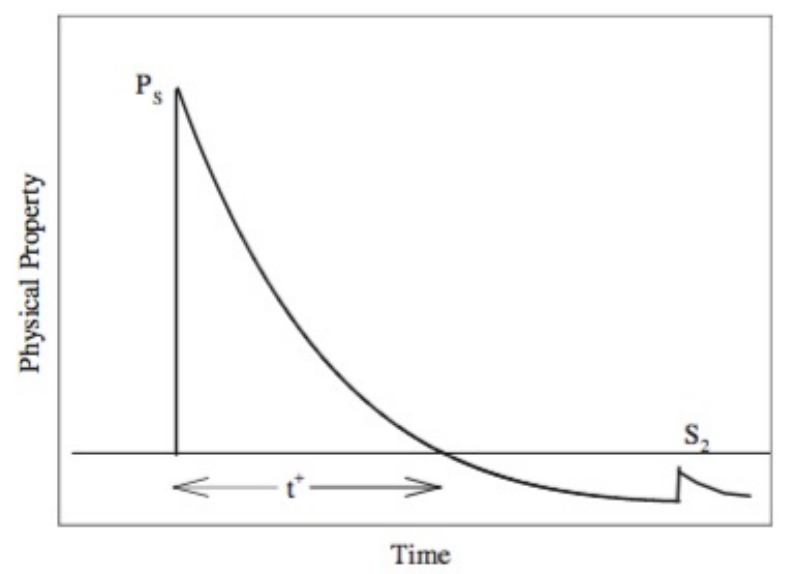

\section{Figure 1.9: Time History for Properties of a Blast Wave[2]}

In figure 1.9 a typical time-history is shown for physical property of the blast wave at a fixed point. $P_{s}$ is the peak value immediately behind the primary shock, $S_{2}$ the second shock, and $t^{+}$the duration of the positive phase. The period when the physical properties are above the ambient value is known as the positive phase, and the period when the properties are below the ambient value is the negative phase. The duration of the positive phase is slightly different for each of the physical properties. Close to the minimum of the negative phase a second shock arrives, produced by the over expansion and subsequent implosion of the detonation products or source materials [2]. This time history is best resembled by the Friedlander wave and studies have been done to attempt to compare how 
well shock tubes can replicate this waveform [13]. The blast wave characteristics produced by the shock tube such as peak overpressures, decay time, impulse, and acceleration are often compared to typical blast profiles of real explosives [13][14] to determine if the blast being produced is acceptable for the desired field of study. It is not the goal of this thesis to fully analyze the blast wave produced by the shock tube to extent as other studies but rather show that the shock tube produces a blast wave as measured by the Integrated Circuit Piezoelectric (ICP) pressure sensors.

To show a blast wave is being produced, the ICP pressure sensors are placed in two positions with the goal of measuring the overpressure of the blast wave where the overpressure is defined as the following:

$$
o p=p_{2}-p_{1}
$$

Where the pressure in region 2 as shown in figure 1.10 is the pressure jump cause by the initial shock and the pressure in region 1 is ambient pressure.

(A)

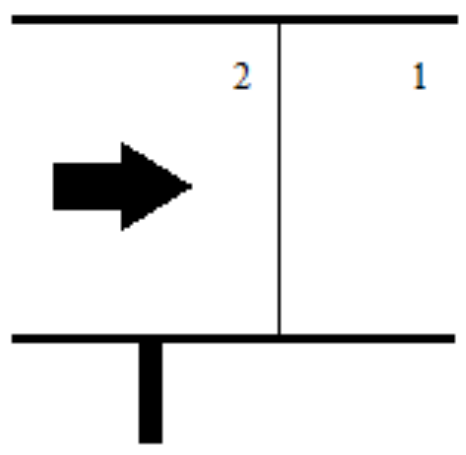

(B)

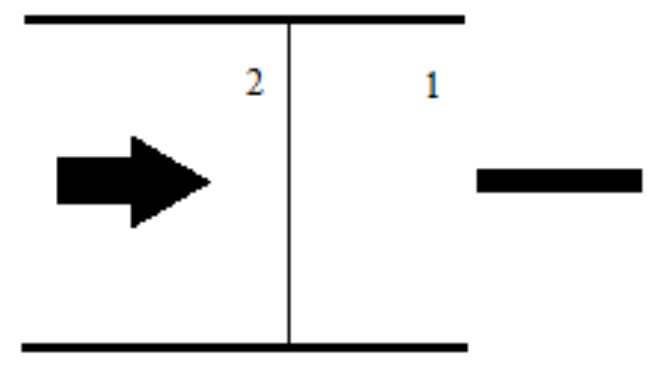

Figure 1.10: Pressure Sensor Positions Relative to the Direction of Travel of the Blast Wave 
In figure 1.10, (A) shows the sensor in a position where it will be known as the Incident (Side-On) Pressure Gauge and (B) shows the sensor in a position where it will be known as the Reflected (Face-On) Pressure Gauge [14]. The incident pressure can be related to the reflected pressure with the following RankineHugoniot relation [12][14]:

$$
p_{R}=2 p_{I}\left(\frac{7 p_{a m b}+4 p_{I}}{7 p_{a m b}+p_{I}}\right)
$$

Where $p_{R}$ is the reflected pressure and it is a function of the incident pressure $\left(p_{I}\right)$ and the ambient pressure $\left(p_{a m b}\right)$. It is important to note that the sensor position shown in (B) is also measuring stagnation pressure. The stagnation pressure is a combination of the overpressure and dynamic pressure of the shock and is measured by inserting a pressure sensing element opposite of the direction of the flow. Placing the sensor in the flow will cause a reflected shock and therefore any material will need to pass through the reflected shock before reaching the pressure sensor and will be partially stagnated. With that in mind the total overpressure $\left(o p_{t o t}\right)$ can then be determined using the Mach shock number [2].

$$
o p_{t o t}=p_{a m b} \frac{7 M_{s}^{2}-1}{6}\left[\frac{12 M_{s}^{2}\left(M_{s}^{2}+2\right)}{\left(M_{s}^{2}+5\right)\left(7 M_{s}^{2}-1\right)}\right]^{3.5}-1
$$

It is important to note that equation 1.21 is specific to this set up as the the predicted $M_{s}$ is expected to be less than 2.0681 and assumes $\gamma_{1}=1.4$ [2]. The measurements that the sensors collect will then be compared to the Friedlander wave using the following equation:

$$
p(\tau)=p_{S} e^{-\frac{\tau}{t^{+}}}\left(1-\frac{\tau}{t^{+}}\right)
$$


Where $p_{S}$ is the peak pressure, $\tau$ is time and $t^{+}$is the duration of the positive phase. The following figure shows the waveform equation 1.21 produces for a $p_{S}$ of 30 psig and $t^{+}$of 5 milliseconds.

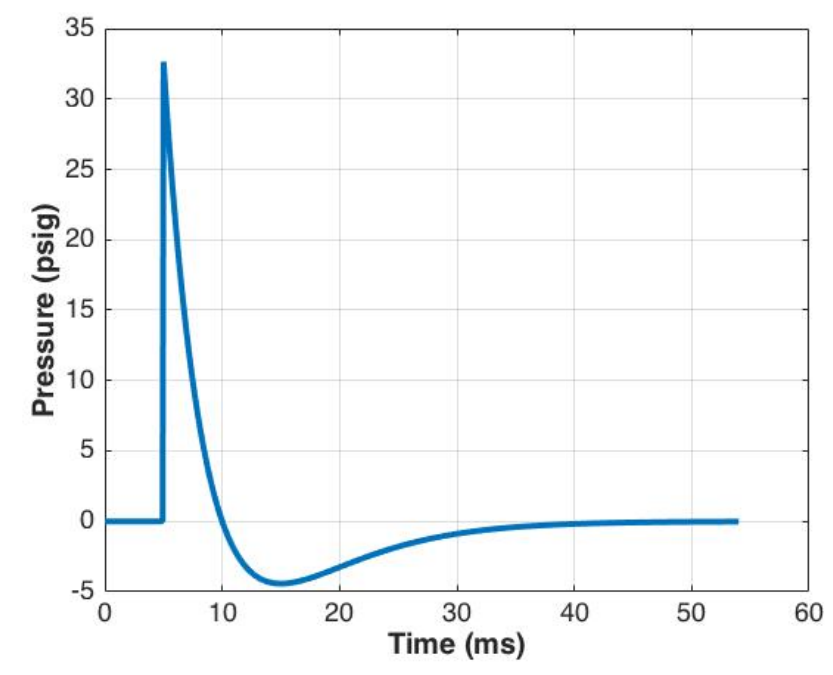

Figure 1.11: Theoretical Friedlander Wave

\subsection{Exit Flow of an Open End Shock Tube}

At the exit of the tube there are further flow features that occur that must considered to understand what is happening in this region. Figure 1.12 is an image obtained using PIV at the exit of an open ended shock tube. As the shock wave exits the tube it diffracts and this causes a vortex ring to form that is followed by a high speed jet [3]. A vortex ring is defined to be a bounded region of vorticity in a fluid in which vortex lines form a closed loop [15]. While a lot of research as been done on incompressible flow vortex rings there has been not much attention to the flow field that is produced by the shock tube although recent efforts have been made to further understand the unsteady flow [3][15][4]. As mentioned before the goal of this thesis is to build and instrument a shock 
tube but in order to fully understand the measurements it is necessary to touch base on this subject to demonstrate the different features the pressure sensors will detect especially those placed at the exit of the tube (See Apparatus and Set Up Chapter for more details).

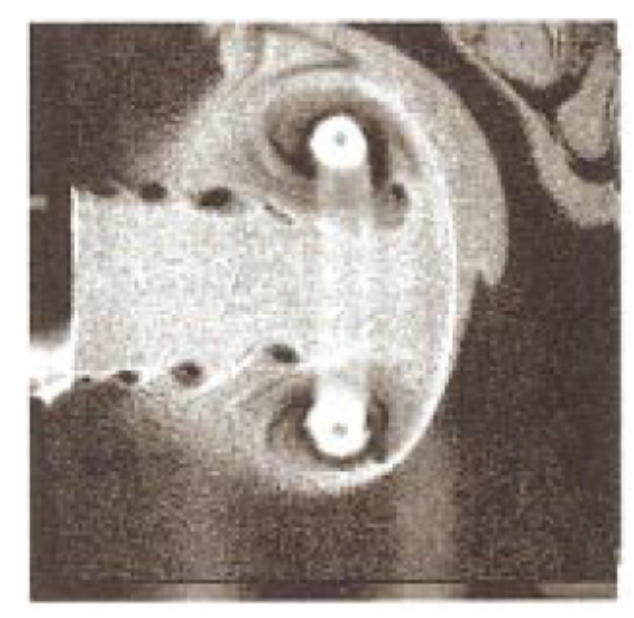

Figure 1.12: Vortex Rings at Exit of Open End Shock Tube [3]
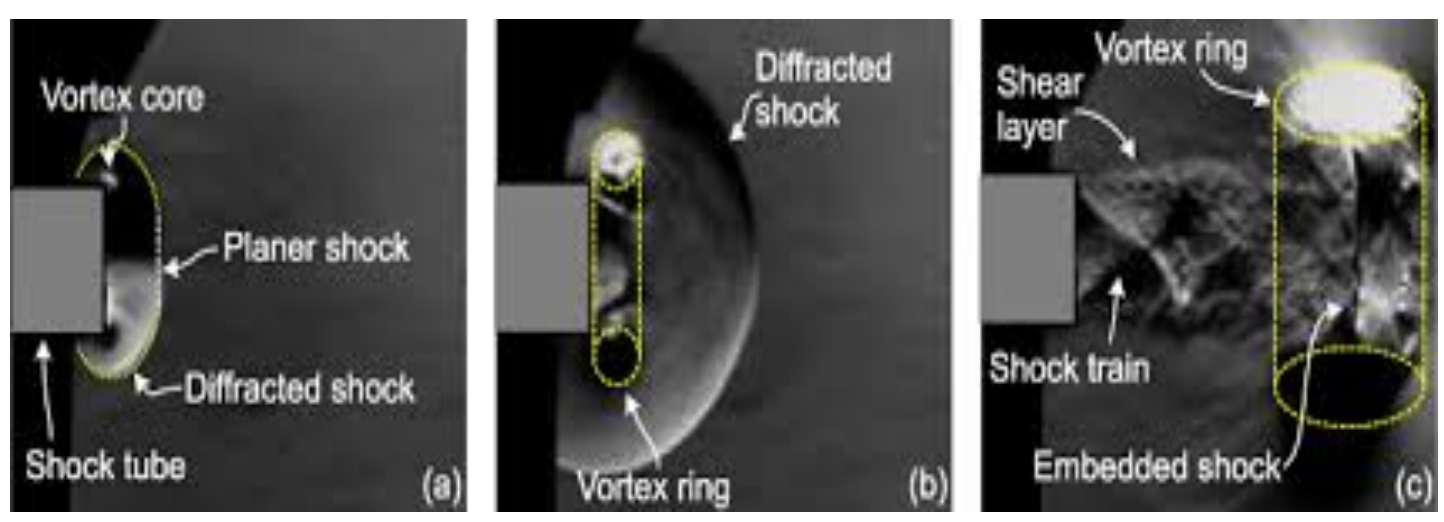

Figure 1.13: Exit Flow Later in Time [4]

Figure 1.13 shows the exit flow with the shock wave propagating out of the shock tube, this image was obtained using Schlieren photography techniques. While in these images the vortex rings are not as clear, the diffracted shock as well as other shock features are clearly visible and denoted in the figure [4]. 
Chapter 2

Design and Manufacturing of Open End Shock Tube

Although the shock tube is simple in nature there were various parameters to be considered which leads to the purpose of this chapter that is to go over how the open end shock tube was designed and how it was put together. This chapter is organized in a manner to first introduce the design requirements set forth to have a clear goal on what the final product was to be. The sections that follow will then go into detail how certain sections were designed, the reasoning for certain designs, and finally how the component was put together or installed.

\subsection{Design Requirements of the Shock Tube}

The design requirements of the shock tube were as follows:

- The shock tube must be a general purpose open end shock tube but could be adapted to later be turned into a closed end shock tube.

- The shock tube was to fit inside a typical lab space then be moved and stored easily when not in use. In order to add clarity to this requirement a lab space of 10'x 15' was assumed.

- Operation of the shock tube should be simple yet safe to use so that there 
would be no need for extra facilities to be built or special personnel needed.

- Turn around time for the shock tube should be as minimal as possible.

- From the beginning of the project it was determined for data acquisition the shock tube was to use PCB ICP Pressure 113B24 Sensors, the PCB Model 482C05 Signal Conditioner, and the NI PXIe-1041 Digitizer as these were already available.

\subsection{Selecting the Driver Gas of the Shock Tube}

There are two methods to drive the gas in the driver section, it could either be compressed gas or blast driven. The first drawback to having a blast driven shock tube is the use of explosives which require special facilities to handle and store the explosives as well trained personnel[16]. This would deviate from the previously stated design requirements so this method was not considered further. Since the method to drive the shock tube would be compressed gas it became necessary to look at a driver gas that would fulfill the requirements. The purpose for having an open end shock tube would to develop shock or even blast waves fields in the laboratory setting which would mean compressed gas driven shock tube would have some drawbacks since the compressed gas tends to form different wave forms and doesn't accurately produce blast waves from typically explosive devices. Also there is an added "jet effect" that could produce additional forces on a test object subjected to the shock wave. This is a concern mainly for the medical community where they are interested in initial effects of shock and blast fields on biological material [16] but this was not an immediate concern or part

of the design requirements so next step was to select the driver gas. Compressed air was chosen as the driver gas since it is readily available in all the aerospace 
labs at Cal Poly. Also the compressed air lines would allow for driver pressures of up to 135 psia or shock tube pressure ratios of $\approx 9.2$ which would be sufficient.

\subsection{Selecting the General Dimension of the Shock Tube}

The dimensions chosen for the shock tube were done following the lab space requirement as well as published dimension requirements for desirable blast profiles[16]. The dimension requirements suggested that the driven section length be 60 times the length of the diameter and 10 times the length of the of the driver sections. The table 2.1 shows the dimensions decided upon in both English and metric units.

Table 2.1: Shock Tube Dimensions

\begin{tabular}{|c|c|c|}
\hline Part & Dimension (in) & Dimension (mm) \\
\hline Diameter of Shock Tube & 2 & 51 \\
\hline Driver Length & 12 & 30.5 \\
\hline Driven Length & 120 & 3050 \\
\hline
\end{tabular}


While the lab space requirement was set to $10^{\prime}$ x $15^{\prime}$ it was decided to not use the full length of the room as there needed to be some space for clearance at the open end for instrumentation that would eventually be used at the exit. Likewise the longer the shock tube would be the harder it would be to move and store as it would take up a lot of space and it would also increase in weight.

\subsection{Determining the Material and Thickness of Shock Tube Walls}

With the general dimensions decided upon for the shock tube now the material could be chosen which would then directly lead to choosing an appropriate thickness for the shock tube walls. The decision for picking the material came by following design suggestions from early publications that gave practical details about shock tube design [17]. The tube must be rigid enough to not only withstand the pressures which are applied but not vibrate under transient loading. This led to considering two materials to use for the shock tube, PVC and commercially available steel pipe. While PVC would have held up with the correct thickness, this pipe tends to sag for the length that would be needed. So commercially available steel was decided upon as thickness could be used that would not make the tube overtly heavy and would would not sag. In order to determine thickness first the maximum pressure the tube would be exposed to at any given time. The max pressure would be seen in the driver section before diaphragm rupture denoted as $p_{4}$, and even after diaphragm rupture $p_{4}$ would still be the highest pressure. The highest pressure this would be is 120 psig, so in order to calculate the wall thickness, structural equations for thin walled pressure vessels were used to calculate the hoop and longitudinal stresses as shown in the figure 2.1. A vessel can be considered "thin walled" if the wall thickness is one tenth or less than the diameter [18] after going through the calculations and selecting 
a pipe this assumption is shown to be valid.

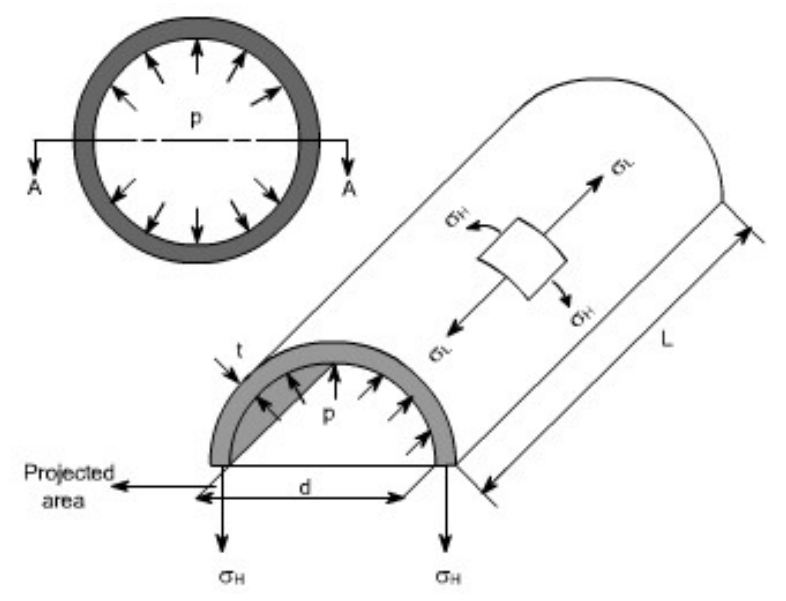

Figure 2.1: Hoop and Longitudinal Stresses in Thin Wall Vessel[5]

The equations to determine the stresses are as follows:

$$
\begin{gathered}
\sigma_{H}=\frac{p d}{2 t} \\
\sigma_{L}=\frac{p d}{4 t}
\end{gathered}
$$

Where $p$ is the internal gauge pressure, $d$ is the diameter of the pipe, and $t$ is the thickness of the walls. By mere inspection it becomes evident that the highest stress is in the direction of the hoop stress (equation 2.1) so it only becomes necessary to make sure this value does not exceed material limitations. The material chosen to make the shock tube out of was commercially available steel tubing and the issue that arises from purchasing these types of materials is that there is no certification on its yield or failure strength but this was mitigated by looking up the material properties of different steel tubing to establish a range of the materials strength. Steel has a yield strength between 30 ksi - 55 ksi (206.8 $\mathrm{MPa} 379.2 \mathrm{MPa}$ ), in the design calculations the lower limit for yield strength 
was used to determine whether or not the shock tube would hold up. Equation 2.1 can be rearranged to solve for the minimum thickness as shown:

$$
t_{\text {min }}=\frac{p d}{2 \sigma_{H_{\text {steel }}}}
$$

From the stress calculations it was determined that a minimum thickness of 0.004 in $(0.102 \mathrm{~mm})$ would be the point of yield for the shock tube (See Appendix A for sample calculation). Based off this information and searching commercially available steel tubing while trying to maintain cost in mind it was found that steel tubing with a thickness of 0.125 in $(3.18 \mathrm{~mm})$ could easily be obtained for the lengths required, likewise steel flanges and caps were also readily available and these would have to be used to piece the shock tube together. This wall thickness for the shock tube gives this part of the tube a Factor of Safety of 31.

\subsection{Sealing the Shock Tube}

In order for the shock tube to operate properly it must be free of leaks[17]. The first place where leaks were considered is at the location of the diaphragm where the driver and driven tubes are connected. Since the driver and driven section are connected with flanges the number of bolts needed to maintain this connection is calculated using the following diagram as a guide. 


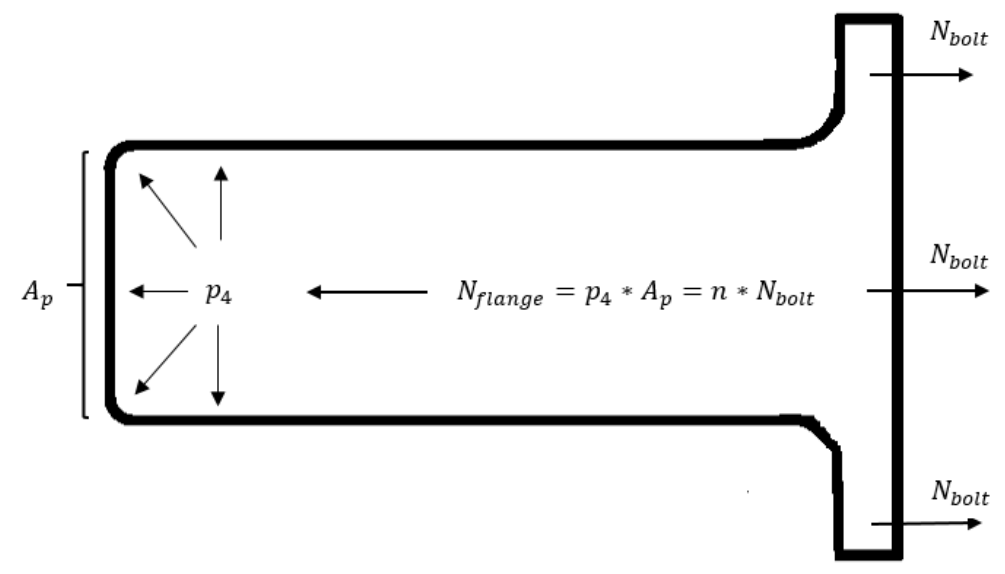

Figure 2.2: Force Diagram for Determining Number of Bolts

Using the following equations to determine the values for $N_{\text {bolt }}$ and $n$

$$
N_{b o l t}=\frac{T}{c M_{D}}
$$

Where $T$ is the torque applied to the bolt to fasten it, $c$ is the coefficient of friction, and $M_{D}$ is the major diameter of the bolt.

$$
n=\frac{N_{\text {flange }}}{N_{\text {bolt }}}
$$

The diagram helps demonstrate how the force acting on the flange is equal to the internal pressure times the projected area and how that has to equal the number of bolts (n) times the force each bolt produces when fully torqued in order to assure the shock tube will stay bolted together during the normal operation. The force each bolt would be dependent for on the amount each bolt was torqued to in order to ensure the bolt did not exceed $80 \%$ of its yield stress. Using a grade 8 steel 14 hex bolts from the recommended torque is 14 lbs-ft $(18.98 \mathrm{~N}-\mathrm{m})$. The value obtain for $\mathrm{n}$ is 1 (see Appendix A for sample calculation) and while theoretically only one bolt is necessary it is important to consider that the force 
needs to be evenly spread out around the flange in order to assure a proper seal, it was first assume 4 bolts holes would help evenly distribute the pressure around the flange but also a 4 hole flange was readily available to fit the chosen pipe. Using the four bolts on the flange gives this part a Factor of Safety of 39.

While assuring the two pieces would stay together would be necessary to seal the tube, a proper air seal would be achieved by either using a gasket or o-ring between the two flanges. An o-ring would require that a proper channel be machined into one of the flanges [19] and because of this the o-ring would not be visible during operation of the shock tube. This would rise as an issue as if there was a failure in the o-ring it would not be immediately noticed until after the flanges were separated. A gasket on the other hand could be squeezed in between the two flanges and made of a larger diameter so that if a failure occurred in the gasket it would be noticeable. Also while machining a channel in the flange would have been possible it was much simpler to get a gasket and cut the proper openings hence the gasket was the method chosen. The flanges that were commercially available along with the pipe had a rough surface so for this purpose two extra steps were taken. The first was to line the edge of the flange on which the diaphragm would be on with Teflon tape to make sure the rough edge would not shear the diaphragm. Second a silicone gasket paste was applied in order so fill in the gaps and create a smooth surface as possible between the gasket and the flange. The connection between the driver and driven tubes is seen in figure 2.3. 


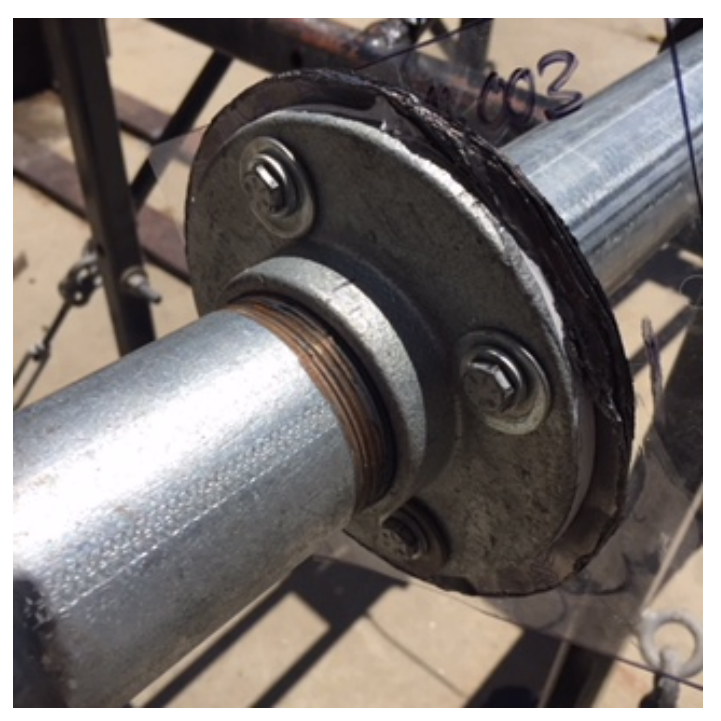

Figure 2.3: Flange Connection with Gasket and Diaphragm

As for the remaining connections since the shock tube is being filled with compressed air it was just necessary to follow procedures for properly sealing air connections by using Teflon tape on all the threads. To assure this set-up did provide a proper air seal a test was performed which is further outlined in the Experimental Methodology and Procedure chapter.

\subsection{Diaphragm Selection}

For simplicity it was decided that it would be best to use a diaphragm that would spontaneously burst which leads to deciding what material would be best for this method of producing the shock. With compressed air chosen as the driver gas and considering that there would not exist high pressure differences between the driver and driven sections it would be best to employ non-metallic diaphragms. The two most common types of these kind of diaphragms are those made of Cellophane and Mylar. The difference between these two is that Cellophane is considered a 'shatter' type while Mylar is considered a 'tear' type diaphragm [6]. 
The importance of considering this since this will be a spontaneous burst shock tube a shatter type diaphragm would lead to fragments being introduced into the tube that would interfere with any other experiment that would follow unless the tube was cleaned out [6] this would lead to longer turn around times for the shock tube. Mylar does not leave fragments but has a disadvantage in that it would have a longer mixing length (see figure 2.4).

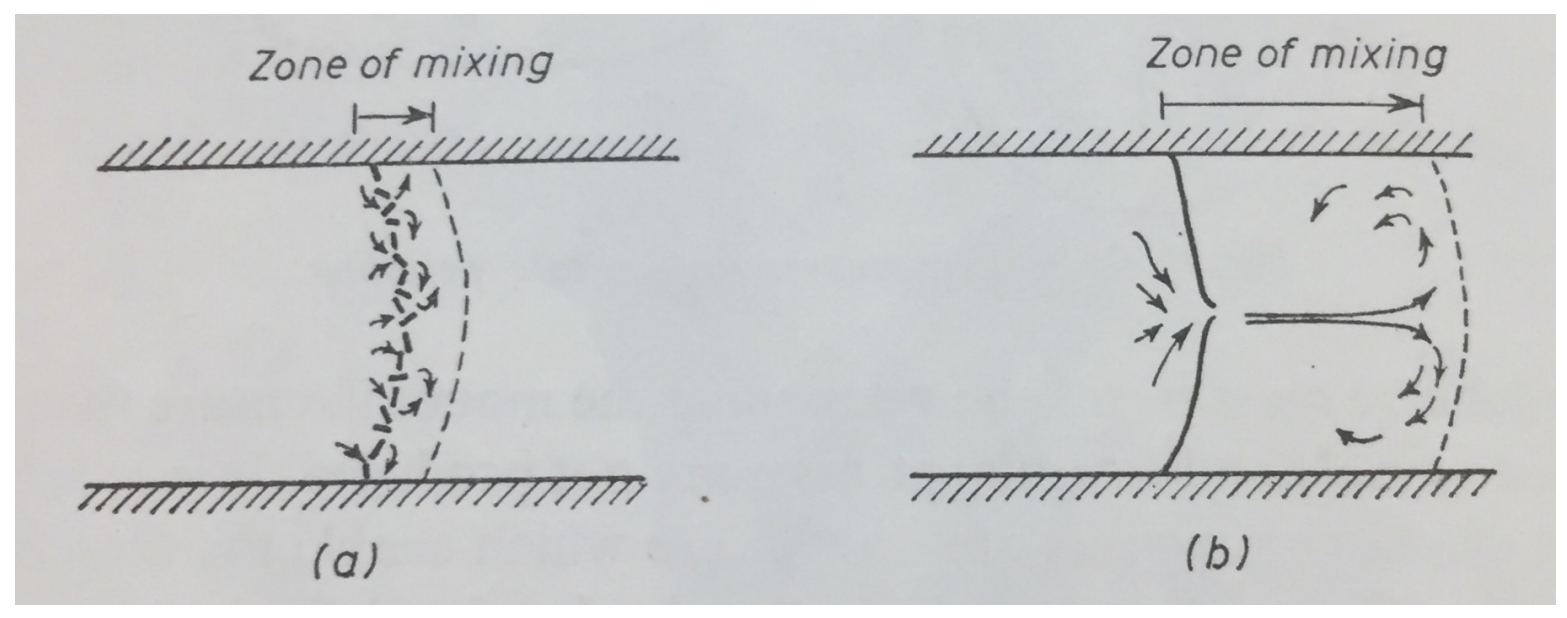

Figure 2.4: Appearance of Flow following Diaphragm Rupture [6]

From figure 2.4, (a) is for a 'shatter' type material and (b) is for a 'tear' type material. This extended region that exist for an appreciable time acts like a nozzle producing a jet in high pressure into the low pressure region and will cause the actual shock velocity to deviate even more from the predicted value [6]. Considering that for compressed gas shock tubes Mylar diaphragms have shown to be effective and practical to use for the application [3][16] and that the tube will be characterized to determine the actual shock velocities and strengths Mylar is chosen to be material for the diaphragm. 
To determine the thickness of the Mylar diaphragms the following equations is used to get a rough estimate of absolute pressure $p_{4}$ in the driver of when the diaphragm will burst.

$$
p_{4}=\frac{2 t \sigma_{u l t}}{D}
$$

Where $t$ is the thickness, $\sigma_{u l t}$ is the ultimate tensile strength of Mylar, $D$ is the diameter of diaphragm under stress. It is important to note that this equation was developed using values at which Mylar typically burst [6] and is only applicable to this set up. Using this equation it was determined that the diaphragm thickness' would be 0.003 in and $0.005 \mathrm{in}$. These were readily available thickness for Mylar sheets and were shown to be suitable for the type of test to they were going to be used (see Appendix for sample calculation).

\subsection{Selection and Installation of Air Valves and Pressure Gauge}

The next step is to provide a way that the air will be supplied to the shock tube. There are a variety of valves that could be used to feed air into the driver section and the valve that was chosen to be used for this step are Schrader valves. These types of valves along with specifications are readily available as they are commonly used for multiple types of air service. The chosen valve has a pressure rating of up to $200 \mathrm{psi}(1379 \mathrm{kPa})$ and the threads were $1 / 8 \mathrm{NPT}$ threads. Normally at this point calculation could be done in order to determine the minimum engagement length of the inlet portion of the threads of the valve to ensure they do not burst out during pressurization of the driver section. Since one of the requirements was to make sure the shock tube was safe to use it was decided to overdesign this portion and the effective engagement length of the 
inlet on the Schrader valve was found on a look up table [7] of American National Standard Taper Pipe Thread.

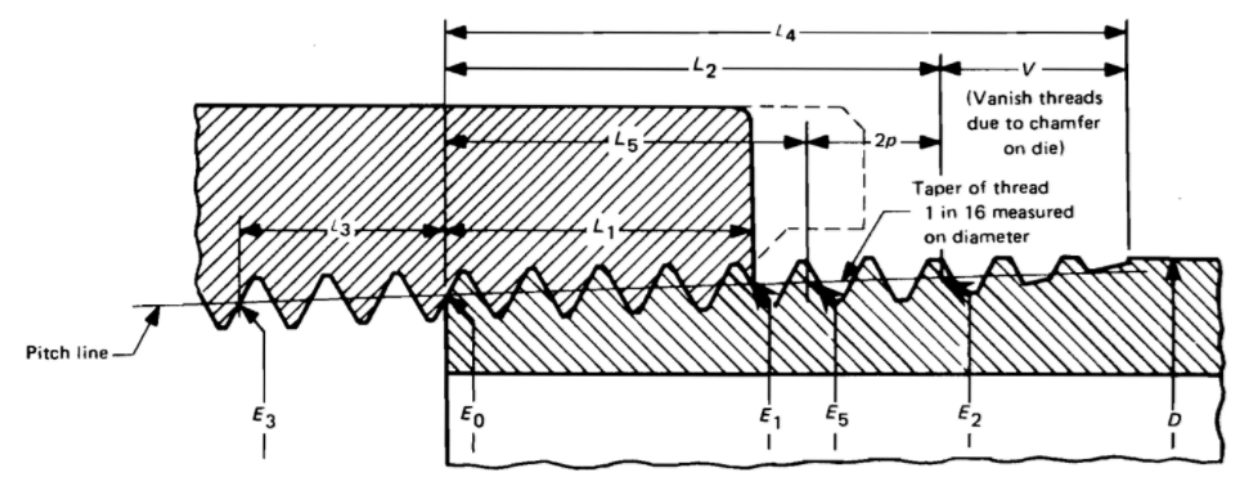

Figure 2.5: NPT Look Up Table Reference Figure [7]

Figure 2.4 is the figure provided with the look up table to show the different dimensions provided by the table. The length that is of importance on the figure is L2 as this is the effective engagement length of the thread. The table indicates that for a 1/8 NPT pipe thread the effective engagement length is 0.2639 in (6.703 $\mathrm{mm})$. Since the valves are rated up to $200 \mathrm{psig}$ then if the full engagement length is used then it can be assumed that the valve threads will not fail under pressure loading as that is how the threads were designed [7]. The only issue that stands is the wall thickness of the chosen pipe is not thick enough to accommodate the effective engagement length so the first option that comes to mind is to obtain a pipe of a thickness that will fit the engagement length. A thicker pipe will lead to heavier shock tube hence making it hard to move, but more importantly the additional pieces needed to put the shock tube together would need to be either manufactured or special ordered. The other option is that material could be added to increase the thickness of the pipe in a localized manner while still maintaining the pipe's structural integrity and then still have the option of using parts that are commercially available. Once the extra material was added on, 
the necessary holes were tapped into the driver tube so that the valves could be installed. To install the valves first Teflon tape was on the threads as an extra measure to keep a good seal which would be aided by the cambered design of the NPT threads [7]. Next the valves were hand tightened into the holes made into the shock tube, then tightened by a wrench until all the threads were fully engaged. Figure 2.6 shows the valves after being installed.

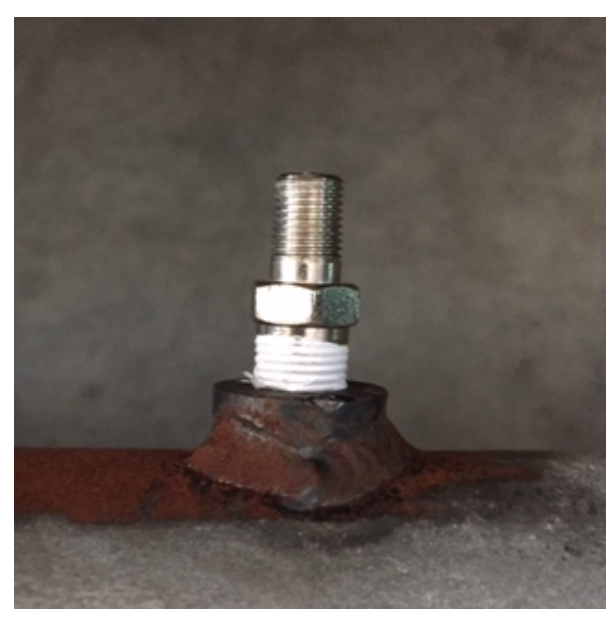

Figure 2.6: Schrader Valves Installed on Shock Tube

To measure the pressure in the driver section it was decided it would be best to have two methods. One way would be to use one of the ICP pressure sensors (the installation of the sensor is discussed in the next section) and the second way would be using a pressure gauge. Bourdon pressure gauges have been used extensively in measuring pressure in compressed air tanks so this was the obvious choice to make. The gauge selected in the end was also rated to work up to 200 psig and had the same threads as the Schrader valves (1/8 NPT) so this would allow for the same installment procedure to be used as the air supply valves. With the chosen air valves and gauge the Factor of Safety for these parts is 1.7. 


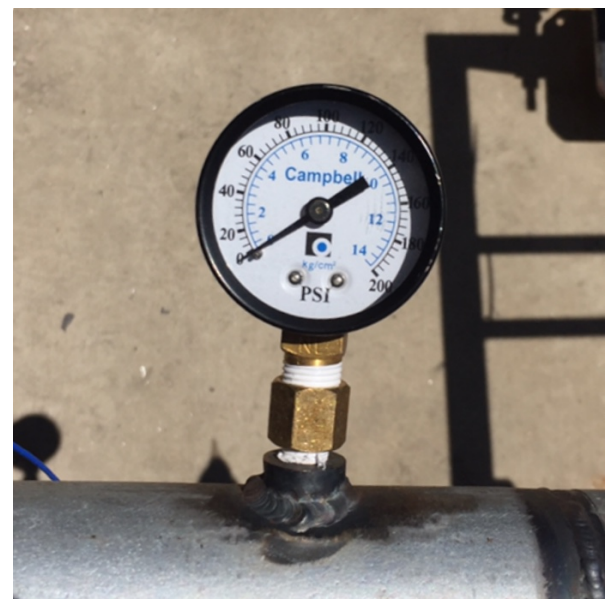

\section{Figure 2.7: Bourdon Pressure Gauge Installed on Shock Tube}

\subsection{Making of Sensor Fixtures and Installation}

There are three locations where the sensors needed to be installed in order to get pressure measurements along the flow of the shock and at the exit of the shock tube. In order to get pressure measurements along the flow, the pressure sensor would need to be mounted on the shock tube and flush with the inner surface. With the given pressure sensors the manufacturer PCB provides plenty of details on various methods to install the sensor, it was decided for ease of manufacturing the sensors would be mounted on the tube with a 062A01 Adapter (see Appendix for the installation drawings). Since the manufacturer's recommended thickness did not match the thickness of the pipe once again material was added in a localized manner as with the air fill valves and this is shown in the figure 2.8. 


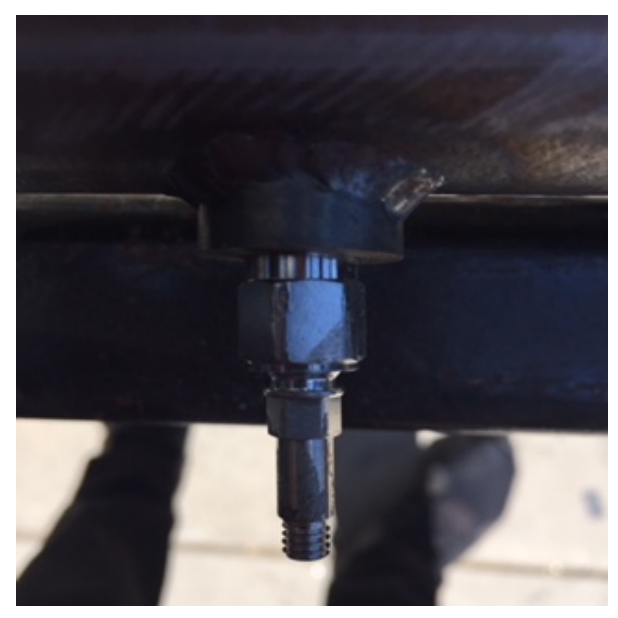

Figure 2.8: Piezoelectric Pressure Sensor Installed on Shock Tube

At the exit of the shock tube there isn't anything physically to install the sensors so in order to collect measurements at the exit the sensors were put in a fixture then attached to a test stand.

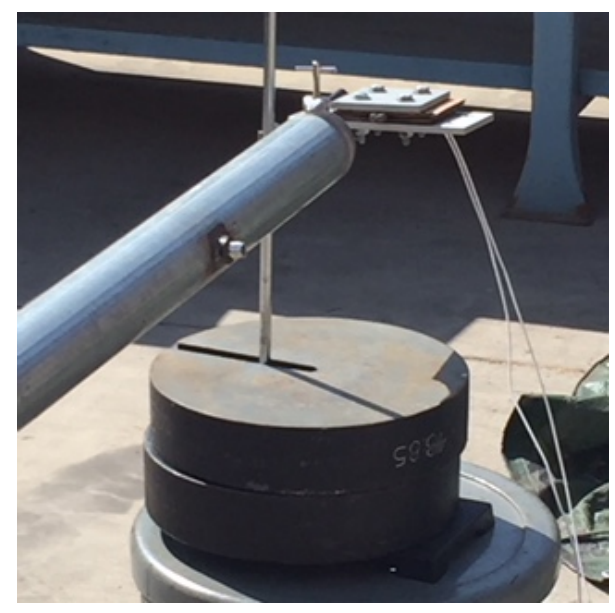

Figure 2.9: Piezoelectric Pressure Sensor at the Exit of the Shock Tube

This set up would allow for the pressure sensors to be fully secured yet allow for the height of the sensors to be adjusted as well move the pressure sensor downstream from the exit of the shock tube as desired. A problem that arose from this set up was the realization that the test stand arm was relatively close 
to one of the pressure sensors in certain configurations as shown in figure 2.10 which would interfere with the results by attenuating the shock wave.

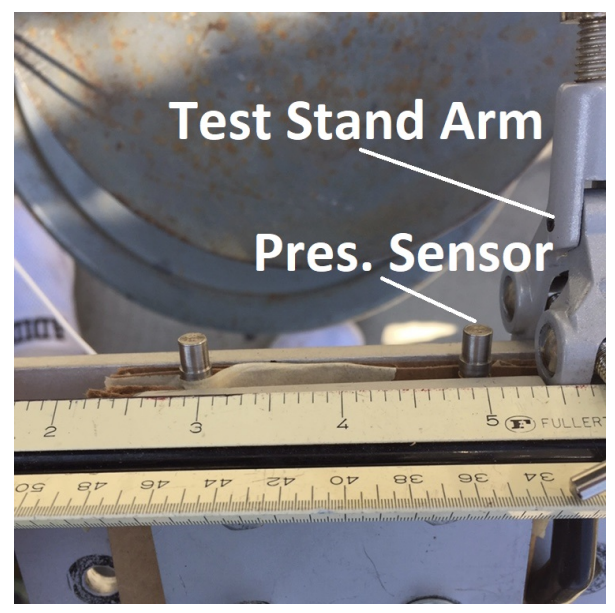

\section{Figure 2.10: Test Stand Arm Interference}

\subsection{Test Stand and Installing Shock Tube}

The determined location where the shock tube would set and fired was decided to be the yard of the Cal Poly Propulsion Lab. The first reason is the shock tube could be set up so that the exit would be facing a blast wall and the second is that the yard is already set up with a test stand. To secure the shock tube to the test stand, hose clamps were used along with rubber sleeves which would help reduce the vibrations. (See figure 2.11) 


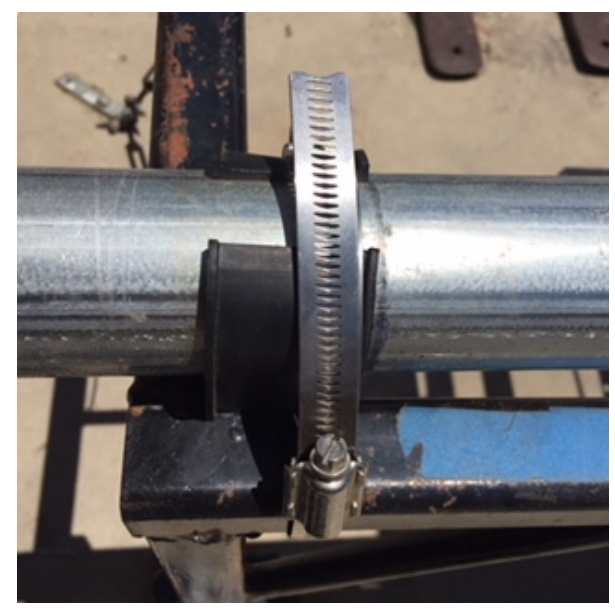

Figure 2.11: Hose Clamp with Rubber Sleeve on Shock Tube

This was done at three location to ensure that the shock tube would be fully secure during its operation. Figure 2.12 shows the shock tube installed on the test stand and set up for a test fire.

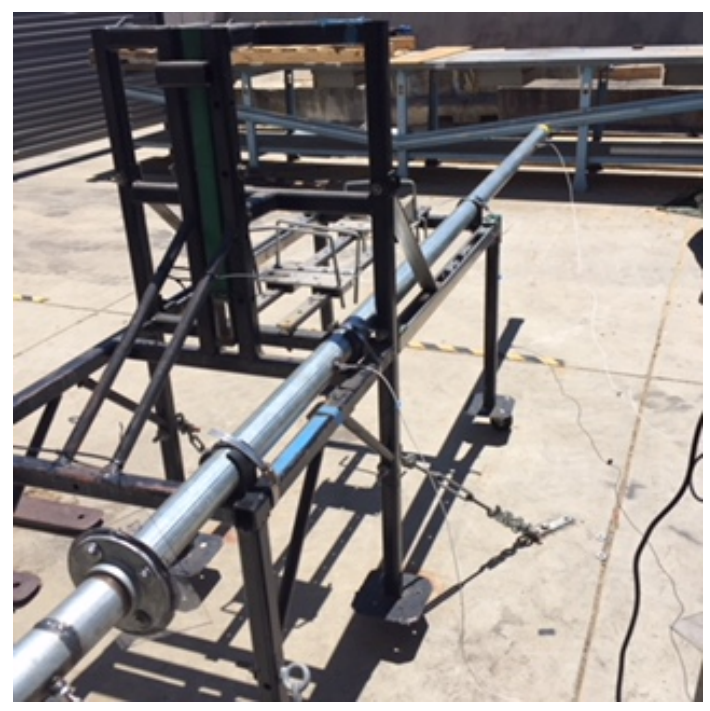

Figure 2.12: Shock Tube Installed on Test Stand 
Chapter 3

Apparatus and Set-Up

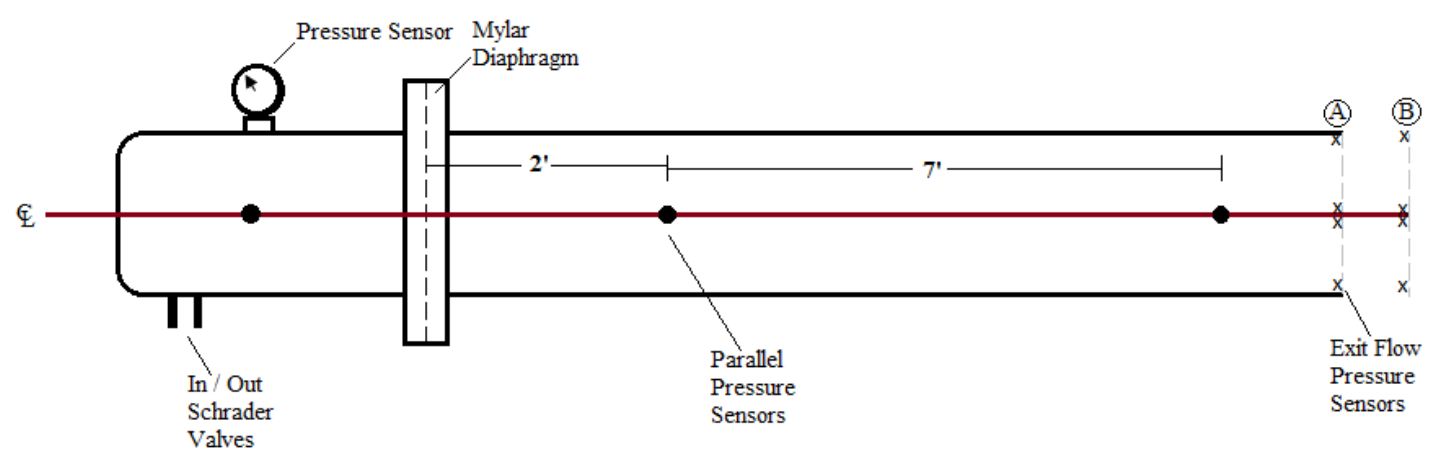

Figure 3.1: Experimental Set Up of Shock Tube

Once put together and sealed, the shock tube was set up in the following manner as shown in figure 3.1 to get it ready for testing. The following sections break down the set up of each major individual component.

\subsection{Driver}

The driver section consists of the steel tube with the addition of the Bourdon pressure gauge, an ICP pressure sensor, and two Schrader valves. Both the pressure gauge and sensor are there with the purpose of measuring the driver gauge pressure until the Mylar diaphragm bursts. The second purpose of the pressure 
sensor is to attempt to capture the expansion that will be formed following the rupture of the diaphragm. The two Schrader valves are installed for practical reasons, one would serve as an inlet valve the other as an outlet valve to empty the driver tube of any compressed air without bursting the diaphragm.

\subsection{Driven}

The driven section consists of the steel tube along with two locations with the pressure sensor adapters so that two ICP pressure sensors can be installed on the driven section of the shock tube to measure the pressure jump of the shock wave parallel with its flow or for the blast wave profiling the incident pressure. The distance of each sensor from the diaphragm is predetermined to be able to use this information combined with the time of each pressure peak to then solve for the speed of the shock wave.

\subsection{Diaphragm and Flange}

To close off the tube, figure 3.2 shows the assembly of the two flanges with the positions that the gasket and diaphragm were installed. 


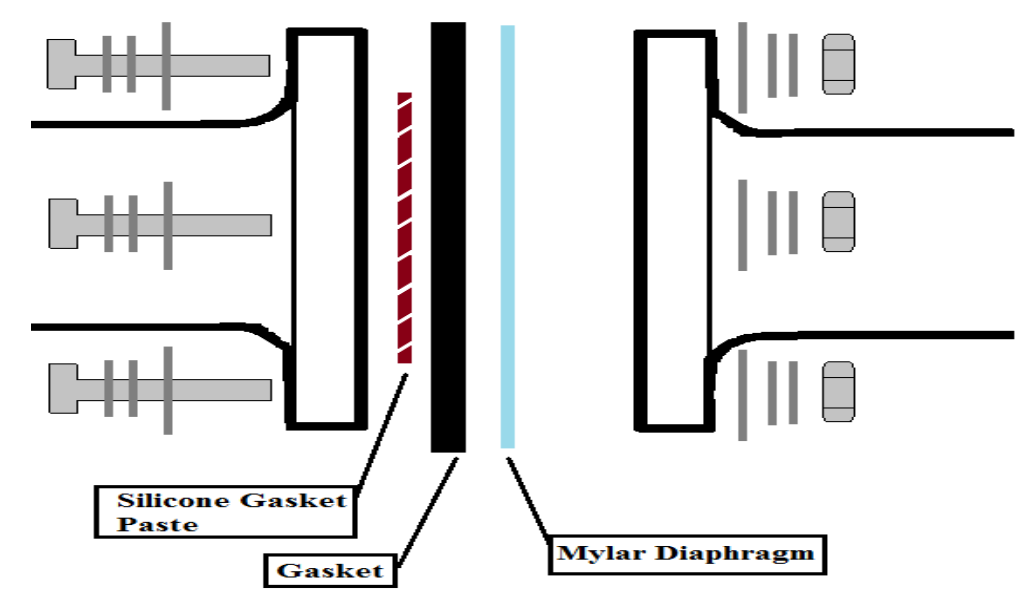

Figure 3.2: Assembly of Flange with Diaphragm and Gasket

First the gasket is placed on so that it can be sealed with the flange of the driven section. Since the flange has an uneven surface a silicone gasket paste is applied to fill in the voids but also act as an adhesive. The diaphragm is then placed on the smooth gasket. The bolts are fed into the holes and on each side 3 washers are used, 2 standard washers and wide flange washer. This is done to allow to use a longer bolt as well as distribute the stress of the preload along a larger surface area. A standard nut is then used to secure the bolts and washers and is torqued to so that the bolt does not exceed 80 percent of its yield stress.

In order to ensure the tube was properly sealed, the shock tube was fully set up with a 0.005 inch diaphragm as this was expected not to burst at the full pressure that could be achieved using the compressed air lines in the Cal Poly Prop Lab. The driver was then pressurized until it reached full the full capacity as indicated by the Bourdon pressure gauge at 120 psig. Then the tube was allowed to sit still and the gauge was observed to see if there was a clear pressure drop additionally soapy water was placed around all the main connections to check for leaks in a visible manner. At the conclusion of this test it was found 
the driver section was fully sealed and did not show any pressure loss within 5 minutes, allowing for the claim that the shock tube is at steady state compared to events that would follow since these are on the order of milliseconds.

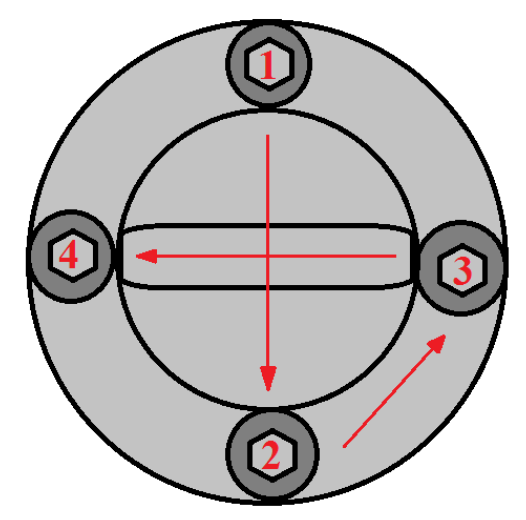

\section{Figure 3.3: Tightening Pattern of Flange Bolts}

Lastly the figure above shows how the bolts were tighten down, instead of going around the flange from the starting bolt in a clockwise (or counterclockwise) motion it was decided to tighten the bolts in a $\mathrm{X}$ formation. This was done with the intention of evenly loading the flange as uneven pressure on the flange could lead to the diaphragm prematurely rupturing due to uneven stresses on it [6].

\subsection{DAQ}

To collect the data a NI PXIe-1071 chassis was used with a PXI-1088 Embedded Controller and three PXI-5114 8-bit $250 \mathrm{MS} / \mathrm{s}$ digitizers for the sensors. The Virtual Instrument (VI) was coded using LabView. 


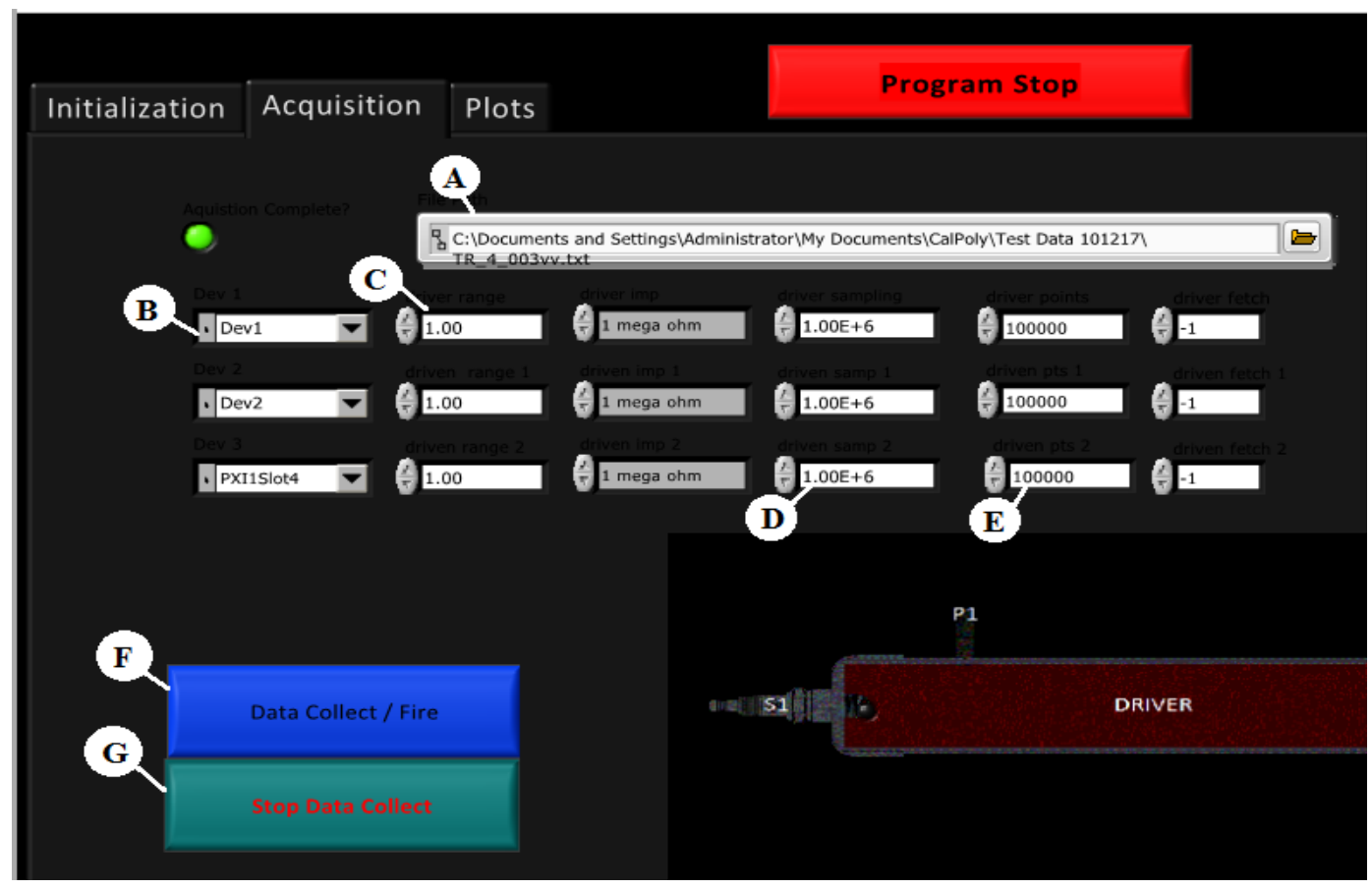

Figure 3.4: Front Page of LabView VI

There are key settings on the VI that are necessary to set up the experiment. Label $\mathrm{A}$ is where the user sets the file path where the text file will be saved, Label B sets up the digitizer card that the user wants to use for a specific channel, Label C sets the voltage (vertical) range, Label D sets up the sampling frequency, Label E sets up the fetch, Label $\mathrm{F}$ is the button to press to start the writing the data to the specified text file and Label $G$ is the button to stop the VI from writing data to specified text file of a particular run.

The architecture of the VI is based on the use of Producer/Consumer loops. The Producer/Consumer design pattern is based on the Master/Slave pattern, and is geared towards enhanced data sharing between multiple loops running at different rates [8]. In other words allows for the DAQ to acquire data at really high frequencies and put in in queue so that the computer has time to write that data to the text file. Also this allows for the DAQ to continuously acquire data, 
meaning if the user presses start the DAQ will continue writing data to the text file until the user presses stop or until the computer runs out of memory.

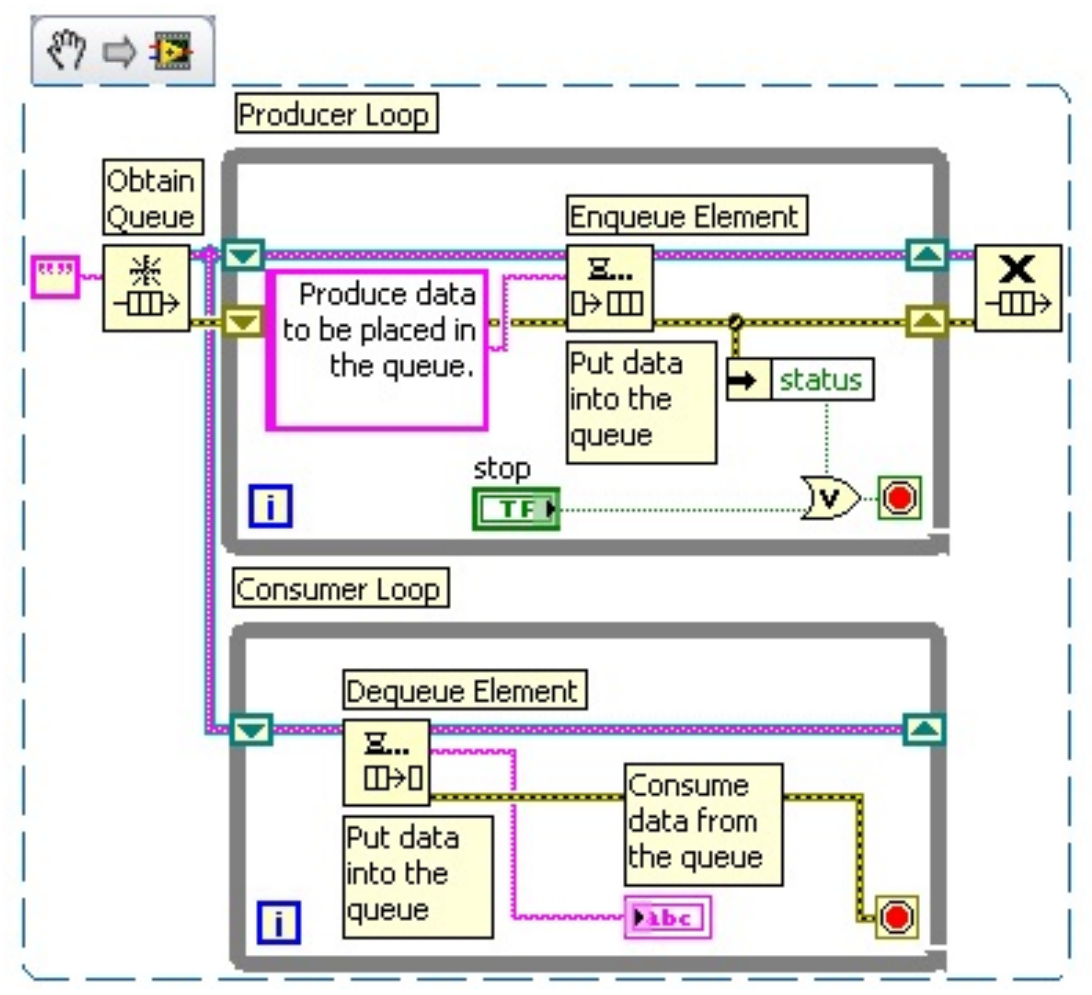

Figure 3.5: Producer/Consumer Design Pattern [8]

The problem that exists with producer/consumer design pattern is that it is not based in synchronization [8] and this leads to a behavior known as a race condition. This means that the DAQ cards do not follow a particular order when they write data to a text file so the data written does not match with real world time. In order to remedy this the following structure in figure 3.6 is used. 


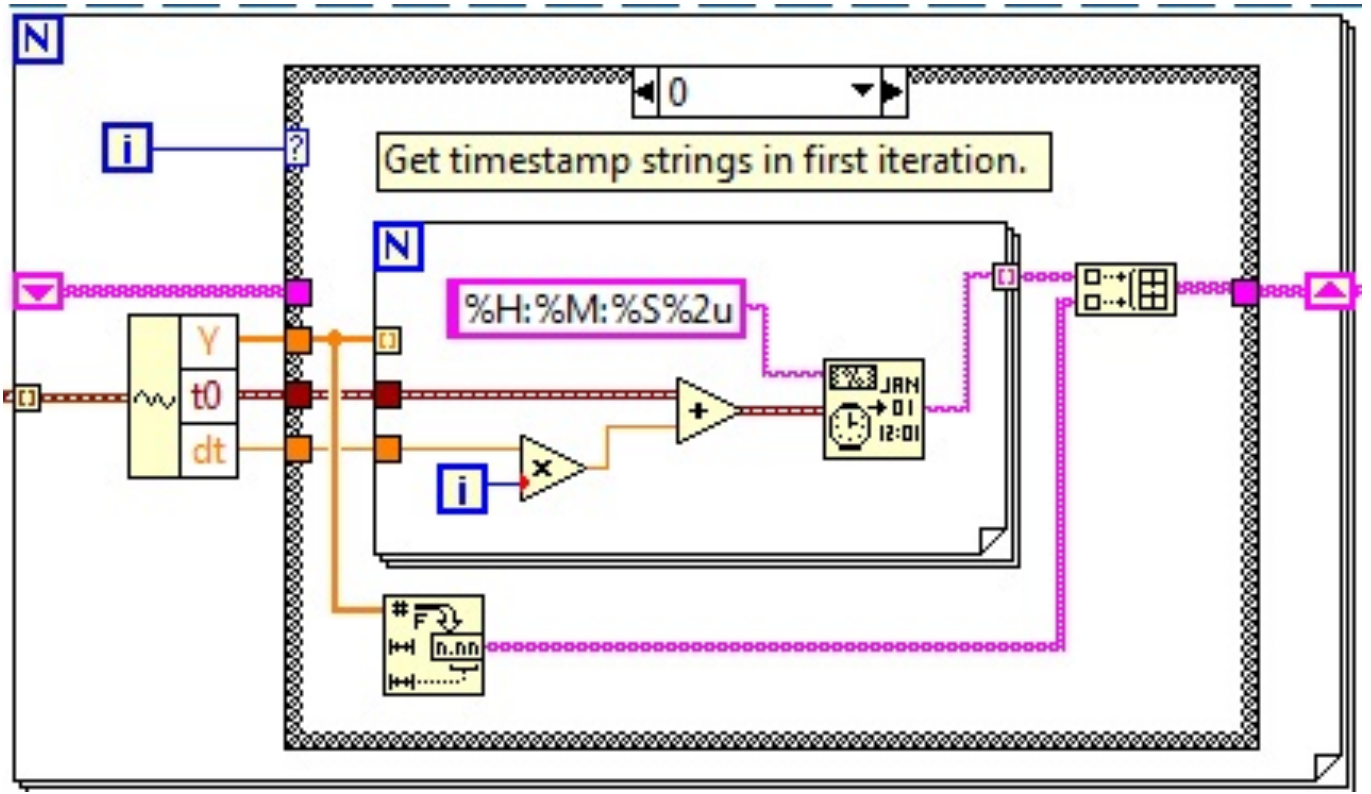

Figure 3.6: For Loop to set Correct Time

This is a for loop which splits the waveform to find the actual real world time in which the card started writing to the file and places a time stamp next each point acquired. This then allows to synchronize the data in post processing as doing this during acquisition would use a significant amount of processing power. 


\section{Chapter 4}

Experimental Methodology and Procedure

Before proceeding into conducting the experiment to characterize the shock tube certain safety procedures were followed, these instructions can be found in Appendix C. The methodology and procedure for major components is outline in the following section for a full test procedure refer to the Appendix C.

\subsection{Pressurizing the Driver Tube}

To pressurize the driver section an air hose was connected to the air supply line with a Schrader valve adapter. Then the air line was turned on and in order to fill the driver tube the adapter was placed on the Schrader inlet valve and filled in a similar fashion as to putting air in a tire. Air was supplied to the driver tube until the diaphragm ruptured.

\subsection{Sensors}

The 113B24 ICP pressure sensors are connected to the Model 482C05 signal conditioner via low noise cables. The signal conditioner serves the purpose of providing the correct voltage and current to the sensors so that they will operate properly during testing. Since the cables to are not that long the current on 


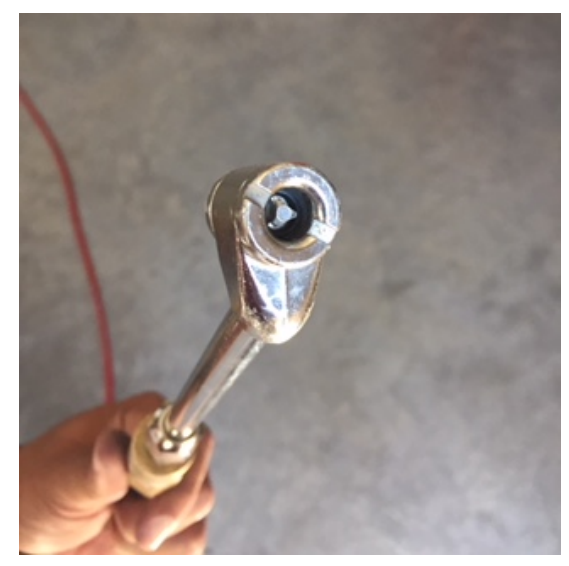

Figure 4.1: Schrader Valve Air Hose Connection

the signal conditioner is left to factory settings as increasing the current is only needed for longer cables (of several hundred meters) and will only lead to a noisier signal. In total there are 3 sensors being used at any given time during testing therefore each sensor is connected to each own individual digitizer card from the signal conditioner using BNC type connectors.

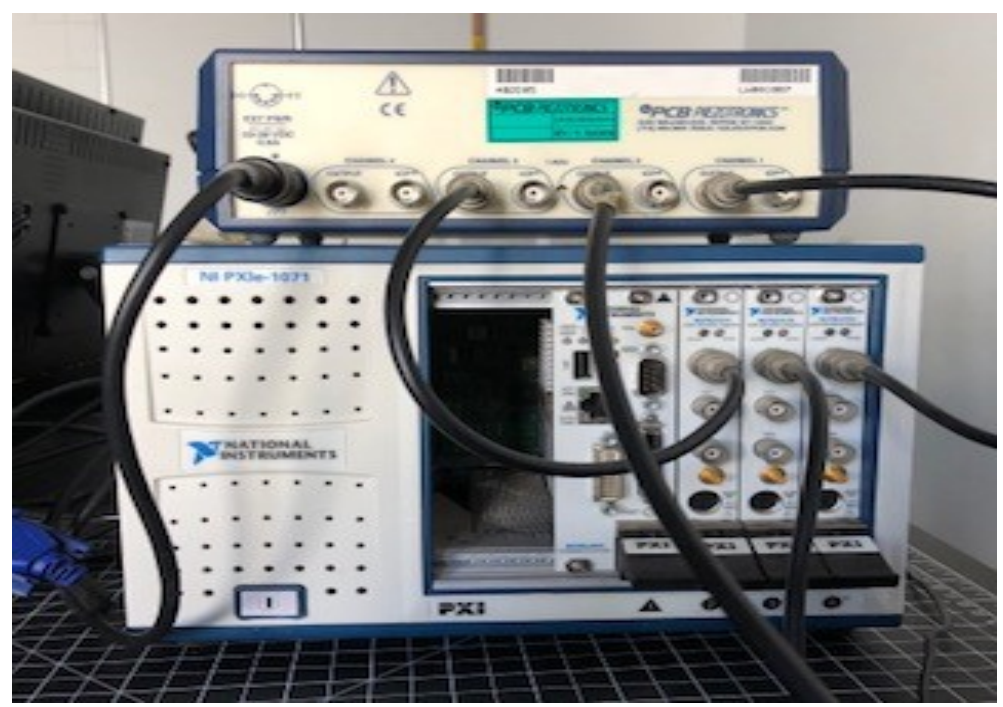

Figure 4.2: Signal Conditioner, Chassis, and DAQ Cards 


\subsection{DAQ Set Up}

The table 4.1 shows the settings for sensors in the DAQ.

Table 4.1: Settings for the DAQ Cards

\begin{tabular}{|c|c|}
\hline Condition & Setting \\
\hline Sampling Frequency & $1 \mathrm{MHz}$ \\
\hline Sensor Range & $0.5 \mathrm{~V}$ \\
\hline Sensor Fetch & 100000 points \\
\hline
\end{tabular}

The sampling frequency is set to make sure that the DAQ is sampling at a rate fast enough to capture the events, the sensor fetch is set to make sure the data points are still in queue and available to written the text file, and the sensor range is set based on test predictions. Since the resolution of the card is 8 bit it is important to set it so that it is not using the full range of the card $( \pm 5 \mathrm{~V})$ rather a specific portion for more accurate results. To see how this value was determined see the Appendix A for a sample calculation.

Once the DAQ has been properly set up through the VI the experiments can begin. Through trial and error, the most effective timing to begin acquiring data is when the driver begins to fill and to continue writing to the file for 45 seconds after the 'bang' from the ruptured diaphragm is heard. This is because the process of writing data to the text file is slower compared to real time so if it is stopped prior to that the sensor data which corresponds to the the shock won't be written to the text file.

\subsection{Characterization of the Shock Tube}

To characterize this shock tube the following parameters were observed in a series of 23 tests with a diaphragm thickness of 0.003 ". 
- Pressure Rise

- Blast Profile

- Shock Velocity

To determine the pressure rise and shock velocity for 7 of 23 tests 2 sensors were mounted on the shock tube so that they are flush with the inner surface of the shock tube wall. This would help show the pressure of the shock changing as it propagates down the tube. Since the distance between the two sensors is known, knowing the time that the sensors first detect the shock the velocity can then be determined (see Appendix C for MATLAB code to calculate velocity). Additionally the 16 tests conducted with the sensors placed at the exit flow are used to determine the pressure rise since this is where the shock wave should be fully developed according to the design of the tube. The reason for this many test is to ensure reproducibility but since there are 2 sensors to use at any given time the sensors were put in 4 different configurations.

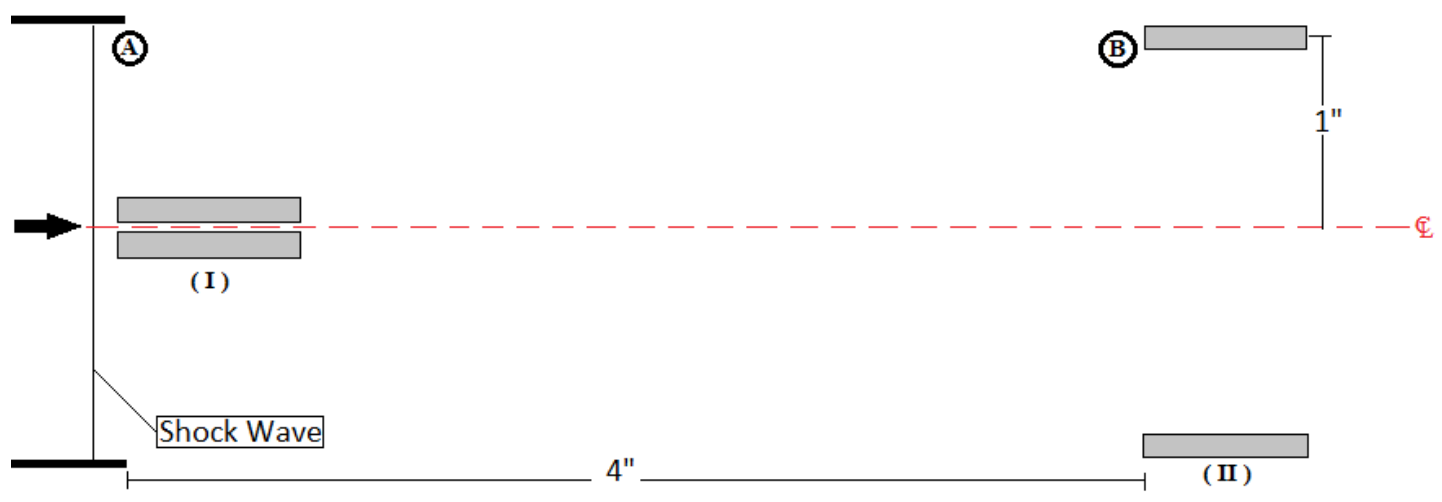

Figure 4.3: Sensor Configurations at Exit

In figure 4.3 the configurations to put the sensors are denoted by AI, AII, BI, BII. Locations A and B refer to the sensor distance relative to the exit of the of 
the tube; in location A the sensors are right at the exit of the tube whereas in location B they are 4" downstream of the exit. Positions I and II refer to distance of the two sensors from the centerline of the tube. Position I puts the sensors right on the centerline and Position II put the each sensors 1" away from the center line. This was done to measure the pressure of the shock at the exit of the tube and see if any effects due to the shock exiting the tube could be detected by the sensors. Also as part of understanding the blast profile moving the sensors downstream can show changes in the pressure rise as the wave propagates from the exit of the tube. 


\section{Chapter 5}

Results and Conclusion

To begin to characterize the shock tube all the data collected was run through a post processing code developed in MATLAB that ran all the data through a 7 th order Savitsky-Golay filter to clean up the noise in the data. This chapter will outline the different parameters that were calculated.

\subsection{Diaphragm Rupture}

After the data was post processed it was necessary to first establish at which pressures $\left(p_{4}\right)$ the diaphragm spontaneously burst. This was done by taking the voltage signal acquired by the pressure transducer in the driver tube and convert-

ing it to pressure using the manufacturers' calibration card value of $200.965 \frac{\mathrm{psi}}{\mathrm{V}}$. In the next figure the pressure data for one of the test runs for the pressure transducer in the driver chamber. 


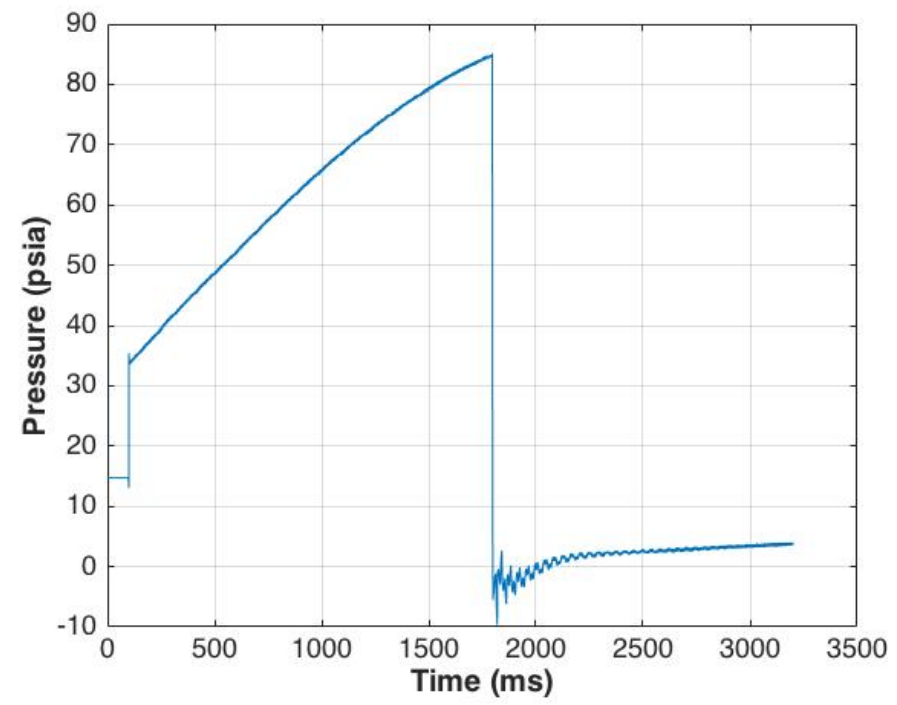

Figure 5.1: Typical Pressure Data of P4 Sensor

The 0.003" Mylar diaphragm was predicted to burst an at absolute pressure of 84 psia in the driven tube and after all the test were completed, the diaphragm was found to burst at an average pressure of 85.91 psia \pm 3.07 psi (one standard deviation). This pressure data also correlated well to the values read off the Bourdon gauge while conducting the test runs.

\subsection{Shock Velocity}

The next parameter to determine for the shock tube was the shock velocity. The reasoning for this is because most of the pressure equations that were used to predict the expected values for the shock wave are dependent on the Mach shock number. With the average driver pressure ratio $\left(\frac{p_{4}}{p_{1}}\right)$ being 5.84 the estimated Mach shock number for the tube is calculated to be 1.72. The theoretical Mach number was measured using 3 known distances and 3 time differences. The results for these measurements are listed in table 5.1 
Table 5.1: Shock Mach Number

\begin{tabular}{|c|c|}
\hline Position & Shock Mach Number \\
\hline Diaphragm to 1st Driven Sensor & $0.591 \pm 0.013$ \\
\hline 1st Driven Sensor to 2nd Driven Sensor & $1.735 \pm 0.008$ \\
\hline Diaphragm to 2nd Driven Sensor & $1.595 \pm 0.005$ \\
\hline
\end{tabular}

On average it was found that the wave decelerated to reduce the Mach number by 0.14 once it was near the end of the tube. This follows the predicted trend that the shock would slow down as it progressed through the tube due to the boundary layer. It is important to consider when comparing theoretical values to the experimental values for pressure data since the lowering of the shock Mach number will reduce the pressure values. Also to further confirm the expected behavior of the shock wave, the Mach number results show an acceleration, a maximum, then a deceleration of the shock wave as it propagates down the tube.

\subsection{Incident (Parallel) Pressure}

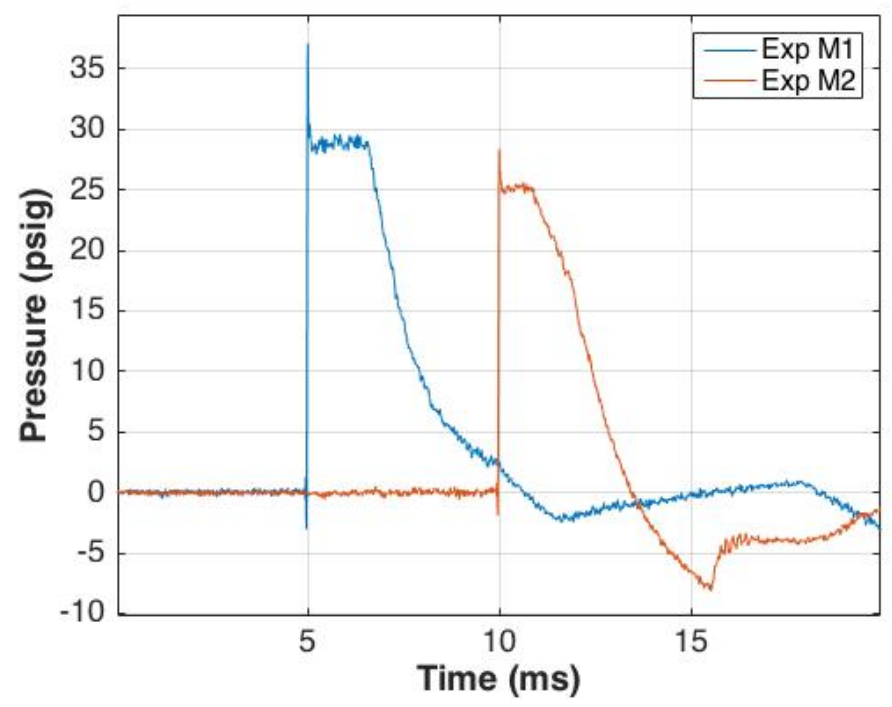

Figure 5.2: Typical Data of Incident Pressure Sensors 
Figure 5.2 shows the data for a test run with the sensors installed flushed to the inner shock tube wall. The measurements have been offset in time for clarity. Before beginning to present the data, the corrections made to the unfiltered signal will be discussed (for a plot of unfiltered data refer to the Appendix D). The unfiltered data was first run through the same filter as the driver pressure sensor, then it was noticed that the voltage data collected had a drift. To correct this an average offset was calculated (see Appendix C for MATLAB code) and applied to the data. Now the next step was to convert the voltage data to pressure but the calibration cards were not found for the these specific sensors so the manufacturers' advertised value was used 200.0 $\frac{p s i}{V} \pm 10 \%$. Now considering the driver pressure ratio and the Mach number using this conversion value did not make sense as it led to values that had more than a $20 \%$ difference from the theoretical pressure rise. So instead it was decided that new conversion value would calculated using $\frac{p_{4}}{p_{1}}, M_{s}$, the theoretical pressure values and the voltage data. The new conversion value to be used with the voltage data obtained with these two sensors was 295.0 $\frac{p s i}{V} \pm 10 \%$. For the specific data set shown in Figure 5.2 the driver pressure ratio was 5.76 (Figure 5.1 is the driver pressure data for Figure 5.2) which lead to a predicted Mach shock number of 1.72 which would be reduced to $\approx 1.58$. This would lead to theoretical absolute pressure values downstream of the shock $\left(p_{2}\right)$ of 47.9 psia and 40.0 psia. Using the data from one test run the experimental values with the new conversion factor were calculated as 47.3 psia and 41.5 psia with percent differences of $1.6 \%$ and $5.9 \%$, respectively. Considering the error band of the pressure sensor these percent differences are completely acceptable with a new corrected conversion factor. Overall it can be said that the shock tube produces an average incident pressure of 47.2 psia \pm 0.4 psi at the first sensor and at the second sensor produces an average incident 
pressure of 41.1 psia \pm 0.3 psi.

The reason as to why the advertised conversion factor is inaccurate and why there is deviation from theory may be due to the fact of manufacturing tolerance and imperfections. Inherently there will be imperfections inside the tube that will deviate from the original value but also considering that this shock tube is made from commercially available steel tubing it is not smooth. Also when the hole was tapped there was no way of verifying that the end of the hole inside of the tube was as smooth possible as it cannot be reached or seen easily. Machine tolerance would lead to the sensor not being flushed with the surface since the tolerance for the taps were $\pm 0.002-0.003 "$. Also the tube is circular which without a doubt makes it unable to mount the sensor flush with the surface. Figure 5.3 shows an estimated position of the pressure sensor inside the shock tube.

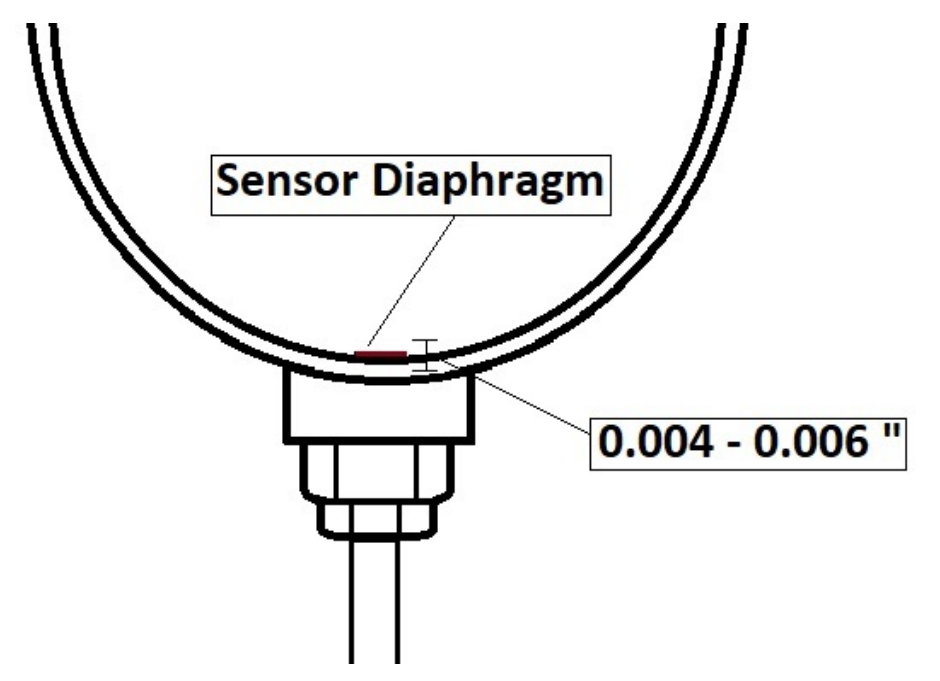

Figure 5.3: Estimated Position of Sensor Inside the Tube

The sensor not being flushed with the surface has the potential to changed the flow field even if it is just by a small amount but also affects the rise time of the sensors which leads to even more potential errors in measurement. 


\subsection{Total / Reflected Pressure Measurements}

Total pressure can be measured by the same type of electronic transducer as used to measure incident pressure, but with the surface face-on to the blast. The total-pressure transducer will initially record the reflected pressure but this will be relieved in a few micro-seconds. This short duration reflected-pressure measurement will appear as a spike at the beginning of the total-pressure measurement, but because of its very short duration may not be a reliable measure of the reflected pressure [2]. The figure below better illustrates the measurements the pressure sensors collect.

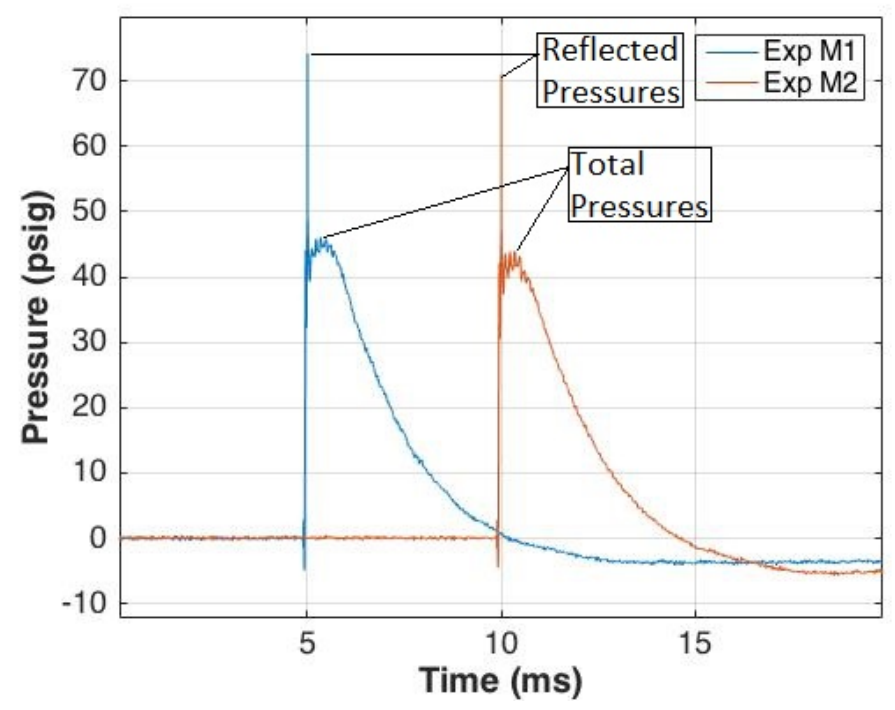

Figure 5.4: Typical Data of Reflected/Total Pressure Sensors

To get a value of what the reflected overpressure will be in this case the following equation is used:

$$
o p_{R}=p_{a m b}\left[\frac{\left(7 M_{s}^{2}-1\right)\left(4 M_{s}^{2}-1\right)}{3\left(M_{s}^{2}+5\right)}-1\right]
$$


Similar post processing procedures were followed for the total pressures measurements as the incident pressure measurements meaning this data set was run through the same filter. The drift was corrected and the data was multiplied by the same corrected conversion factor $\left(295.0 \frac{p s i}{V} \pm 10 \%\right)$ the only difference is after running these data sets through the filter and compared to the unfiltered data it was noticed that the reflected pressure peak was being attenuated significantly so the peak pressure value from the unfiltered data was superimposed on the filtered data set (see Appendix C for MATLAB code). The results for this section will differ since the total pressure and reflected pressure were measured in four different configurations. The only two sets that were compared to the theoretical values are the sets of measurements in position $\mathrm{AI}$ and $\mathrm{BI}$, although no flow visualization was done at exit in the other positions the sensors are meant to be measuring different shock structures at the exit as shown in the introduction section. Also to predict what the pressure jumps will be instead of using the theoretical Mach shock number that is obtained once the driver pressure ratio is known, the Mach number used to compare results from the second incident pressure sensor will be used instead since it is 1 foot from the exit.

Using the average bursting pressure for the diaphragm the expected values for the reflected overpressure was $82.5 \mathrm{psig}$ and the total overpressure was 40.2 psig. From the 4 tests conducted in position AI the average reflected overpressure was found to be $69.8 \mathrm{psig} \pm 6.0 \mathrm{psi}$ and the average total overpressure was found to be $43.6 \pm 4.6 \mathrm{psi}$. The experimental average values compared to the theoretical values lead to percent differences of $15.4 \%$ for reflected overpressure and $8.5 \%$ for total overpressure. The averages pressures from position BI (sample data set is seen in Figure 5.3) were 74.5 psig \pm 6.2 psi and 43.7 psig \pm 7.7 psi. Considering the error band on the conversion factor and the deviation on the average experimental 
pressure these differences are completely acceptable. Furthermore what allows for the data measured to have a larger error band is the fact that as mentioned before considering how short the duration of the reflected pressure is it might not be as accurate. Furthermore, in position AI the reflected wave is a planar wave whereas in position BI it can be expected that the wave has now turn into a circular blast wave which equation 5.1 is intended for. As far as for total pressure measurements there should special care to make sure there is no flow across the surface of the transducer which is achieved by placing the sensor in a pencil or cylindrical mount [2]. Considering that the sensors were not in mount as shown in figure 5.5 below and that both average total overpressures are basically the same indicates precise measurement of this feature.

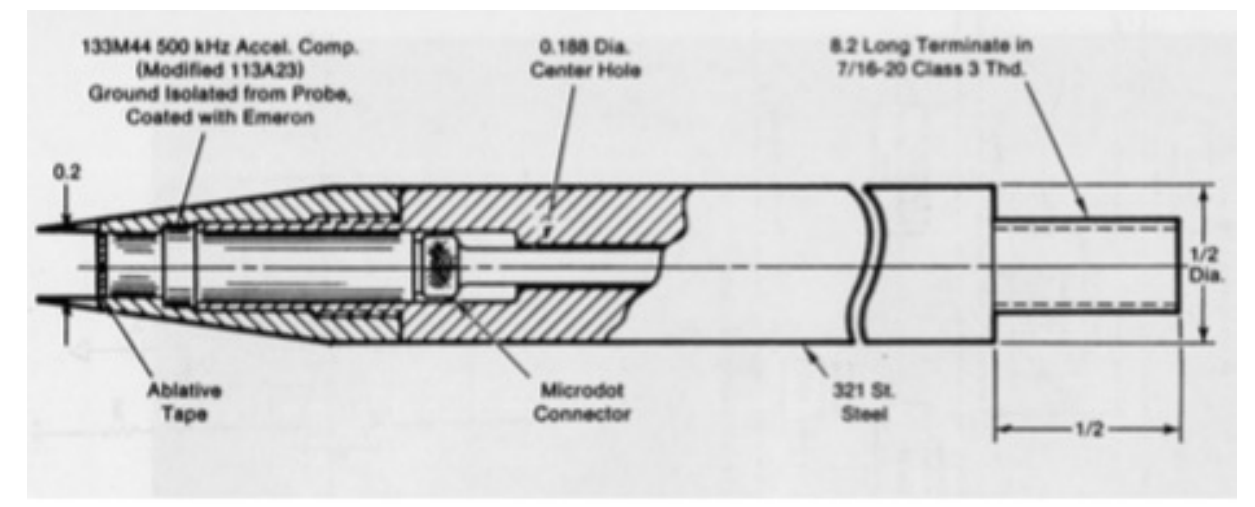

Figure 5.5: Pencil Mount for Total Pressure Gauge [2]

In positions AII and BII while the wave picked up by the sensors the shape of the wave at these positions was completely different. These measurements were likely affected by the fact that they were not aligned with the center of the shock so they would be more affected by a transverse flow. For this reason the data will not be compared to the estimated total and reflected overpressures values. Although putting the sensors in these positions did have a secondary purpose to attempt to capture the flow features at the exit of the tube specifically the vortex 
rings. Figure 5.6 shows the data acquired in position AII and the data has been offset in time for clarity.

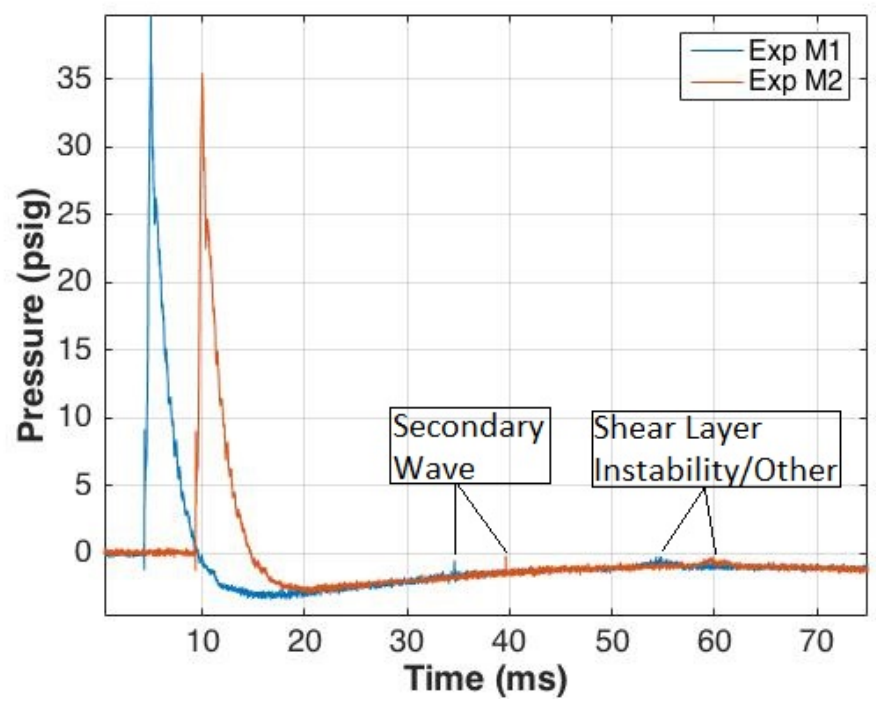

\section{Figure 5.6: Pressure Data for Sensors in Position AII}

Looking at the data which corresponds to the primary wave the shape of the decay is different when compared to the previous positions this is probably due to the fact the sensors are picking up the primary wave off center but also in this particular case as it is diffracting as the wave leaves the tube. There is a secondary peak which is expected but further downstream a small bump is noticed in the data, considering that in this position the sensors are at the exit of the tube it seems it is not likely it is picking the vortex rings but rather either the initial formation of the loop or instabilities in the shear layer. On average the sensors at this location measure a reflected overpressure of $32.1 \mathrm{psig} \pm 1.1 \mathrm{psi}$ and total pressures of 30.1 psig \pm 1.8 psi. Figure 5.6 will show the data for the sensors in position BII and again the data has been offset in time for clarity. 


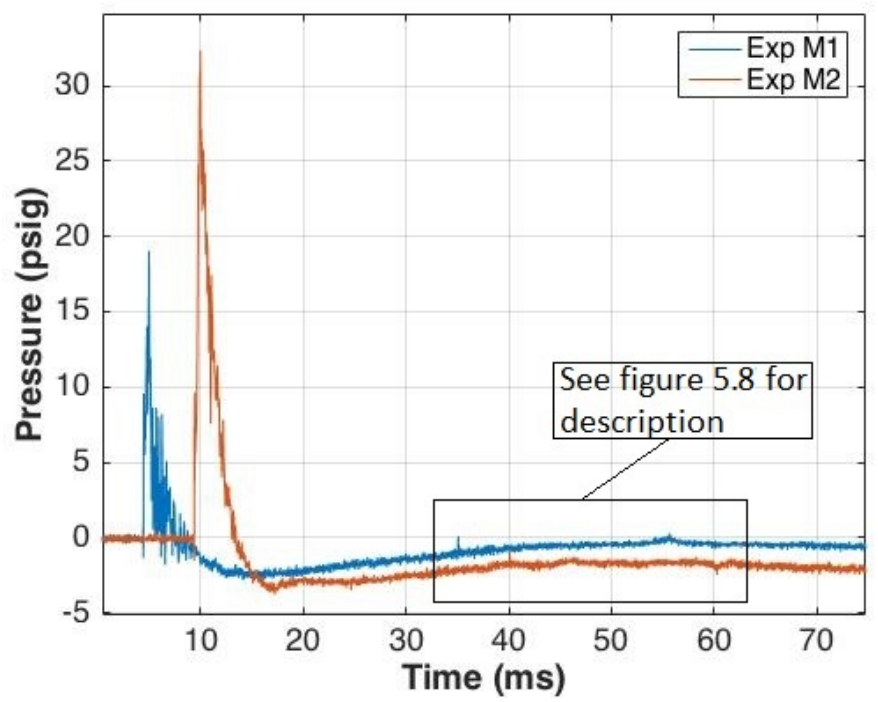

\section{Figure 5.7: Pressure Data for Sensors in Position BII}

In position BII a flaw is noticed of the experimental set in that the bar sustaining the sensors interferes with the flow as very clearly the sensor that was next to the holding arm measures significantly lower pressure values than the one that had no interference. Again the different decay from is seen on these measurements due to the off center measurements of the sensors including the one that had the support arm interfering. On average the sensor not affected by the supporting arm at this location measures a reflected overpressure of 18.6 psig \pm 0.5 psi and total pressures of 15.3 psig \pm 0.5 psi. These sensors also pick up other flow features as shown in figure 5.8.

The first feature seen in figure 5.8 is the secondary wave that follows the primary wave exiting of the tube. After this it would seem that the sensors have picked up the vortex rings passing through. The shape formed makes sense as a vortex loop pressure is highest at the core and lowest at the outer edges of it so the sensors should see a rise to a maximum then decrease back to a level similar to before the vortex passed. Another feature of this measurement that makes 


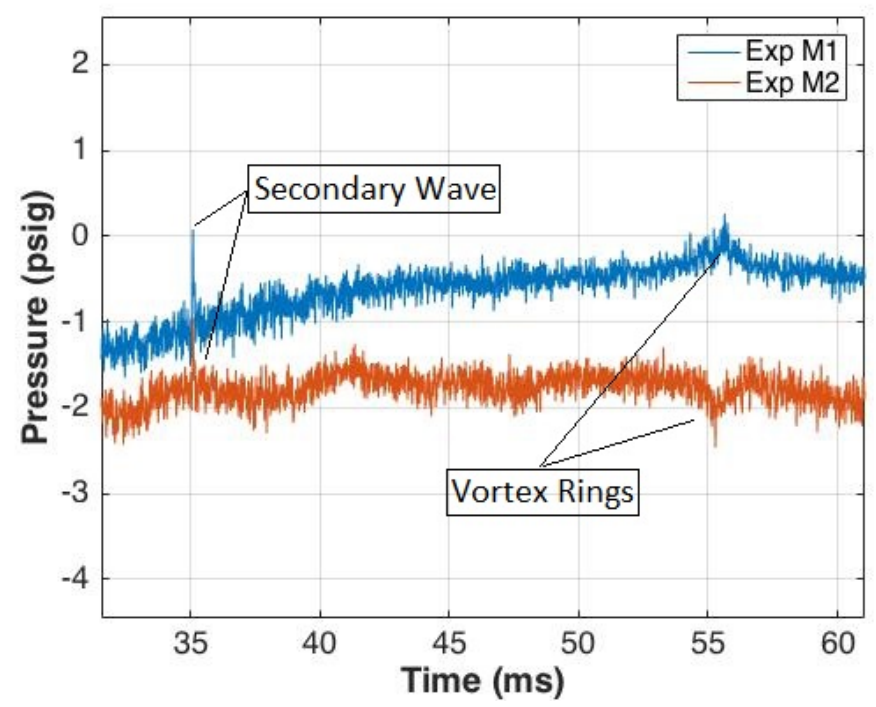

Figure 5.8: Additional Flow Features in Position BII

it possible to be the vortex rings is the shape of the second sensors is similar in shape but in this case the peak is minimum. This due to the fact that there are two vortex loops one will be spinning counterclockwise and the other will be spinning clockwise which will lead to the same absolute pressure but the direction will be different. Now in theory the sensors should have very similar peak values but the reason why this is not the case is because of the interference caused by the supporting arm. Since the actual flow at the exit is not actually visualized it is not possible to actually verify what flow features the sensors are actually picking up in the different positions at the exit of the tube, so it is best to only hypothesize based on previous works. For the same reason it is not possible to quantify what the effect is on the error of the measurements so the acceptable error range was expanded. Table 5.2 summarizes the pressure data from the four different configurations. 
Table 5.2: Reflected/Total Pressure Summary

\begin{tabular}{|c|c|c|}
\hline Position & Reflected Pressure & Total Pressure \\
\hline AI & $69.8 p s i g \pm 6.0 p s i$ & $43.6 p s i g \pm 4.6 p s i$ \\
\hline AII & $32.4 p s i g \pm 1.1 p s i$ & $30.1 p s i g \pm 1.8 p s i$ \\
\hline BI & $74.5 p s i g \pm 6.2 p s i$ & $43.7 p s i g \pm 7.7 p s i$ \\
\hline BII & $18.6 p s i g \pm 0.5 p s i$ & $15.3 p s i g \pm 0.5 p s i$ \\
\hline
\end{tabular}

\subsection{Blast Wave Profiling}

From the exit flow measurements only the data sets obtained position AI and BI will be compared to the Friedlander theoretical wave, the reasoning behind this is because these are the only measurements where the sensors are considered to be centered with the blast wave. The following figure shows the data obtained from the sensors in position BI. This figure shows the time history of the total overpressure with the reflected overpressure data removed.

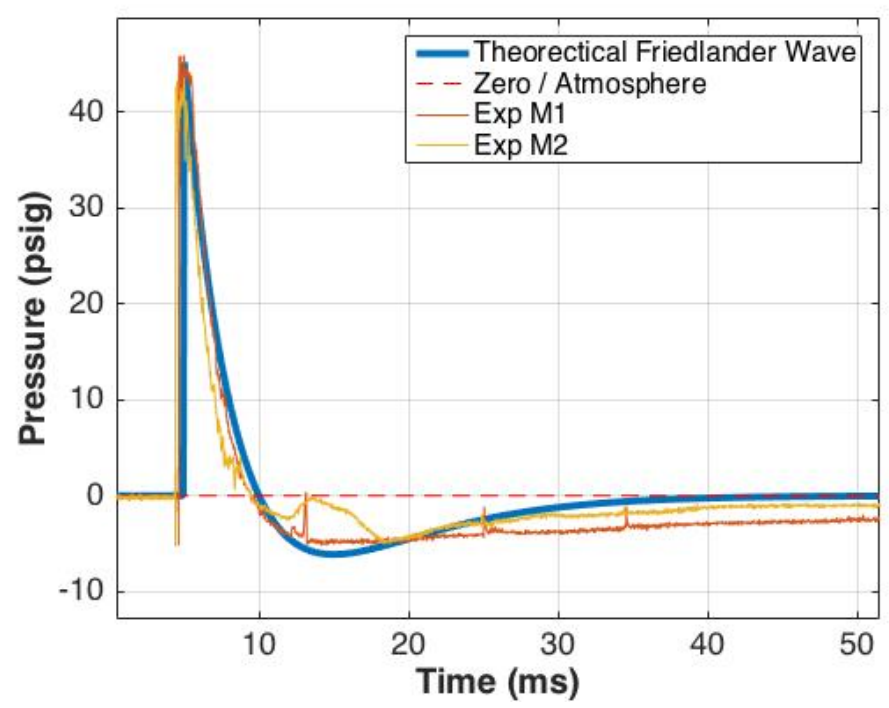

Figure 5.9: Comparison to Friedlander Wave Position BI

In this position the second signal sees a lot of interference from what seems the reflections of the wave off of the first sensor. This is noticed when the signal 
for Exp M1 shows the secondary peak, a significant bump is seen in the Exp M2 signal. Despite this, both signals still show a similar trend to that of the Friedlander wave. Figure 5.10 shows the data acquired at position AI. Once again the figure only shows the total overpressure data.

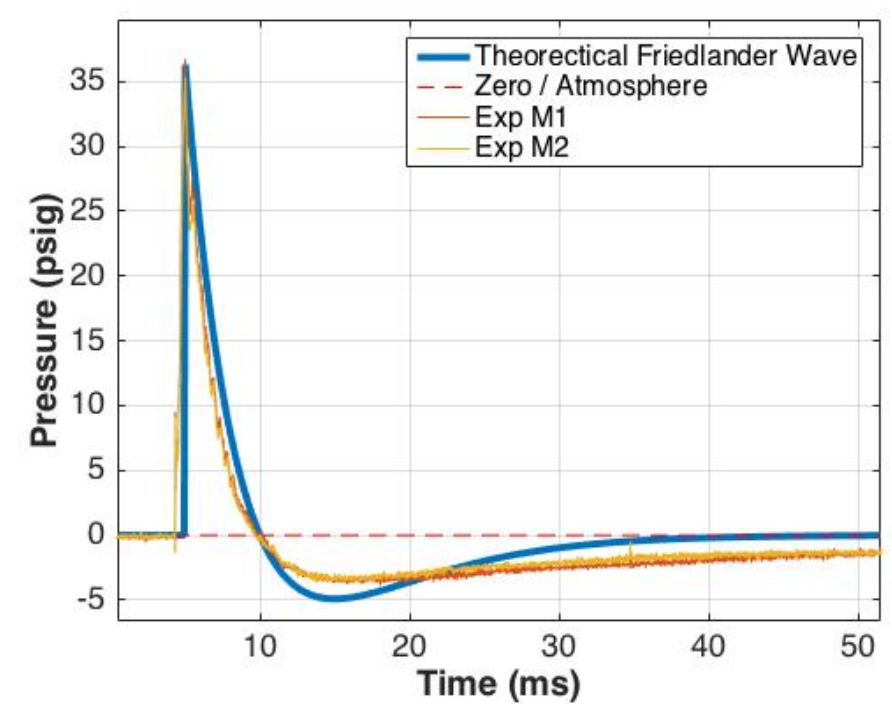

Figure 5.10: Comparison to Friedlander Wave Position AI

Again this data closely follows the Friedlander wave and both signals produce data that has not been affected by the shock structures created due to the proximity of the other sensor. Of course aside from following the trend of the theoretical wave there are other features that must be addressed for these waves to be considered blast waves. In both figures 5.9 and 5.10 the positive phase and the negative phases of a blast wave exist also the secondary wave peaks are also measured near the minimum point in the negative phase as predicted. One trend that is similar in both figures is in the negative phase of the data, that it does not follow the theoretical trend at all in this region. This can be explained by knowing that this is a compressed air blast wave whereas the Friedlander wave deals with waves produced with explosive so the characteristic dip in the nega- 
tive phase is a function of the energy available from the explosives which is not present in the compressed air wave. Of course for completeness the Friedlander wave is also compared to the static pressure measurements obtained from the incident pressure measurements.

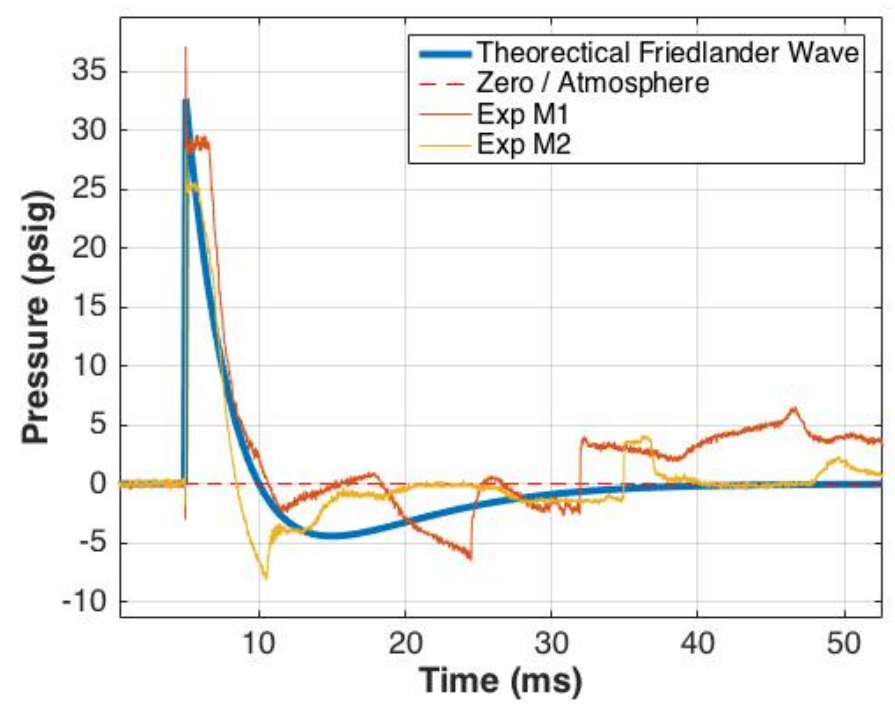

Figure 5.11: Comparison to Friedlander Wave to Incident Pressure

From figure 5.11 it is clear that the signal is affected by a secondary feature as the wave reaches its negative phase. The cause behind the disturbance in the signal is believed to be due to the sensors attached to the tube, they are capable of picking the effect of the diaphragm bursting and how that translates down the shock tube walls. Figure 5.12 shows a time history of all the signals collected in the incident pressure runs. While the decay of the incident pressure is seen due to the extensive nature of the deviation of the data an appropriate comparison to the theoretical wave cannot be done. 


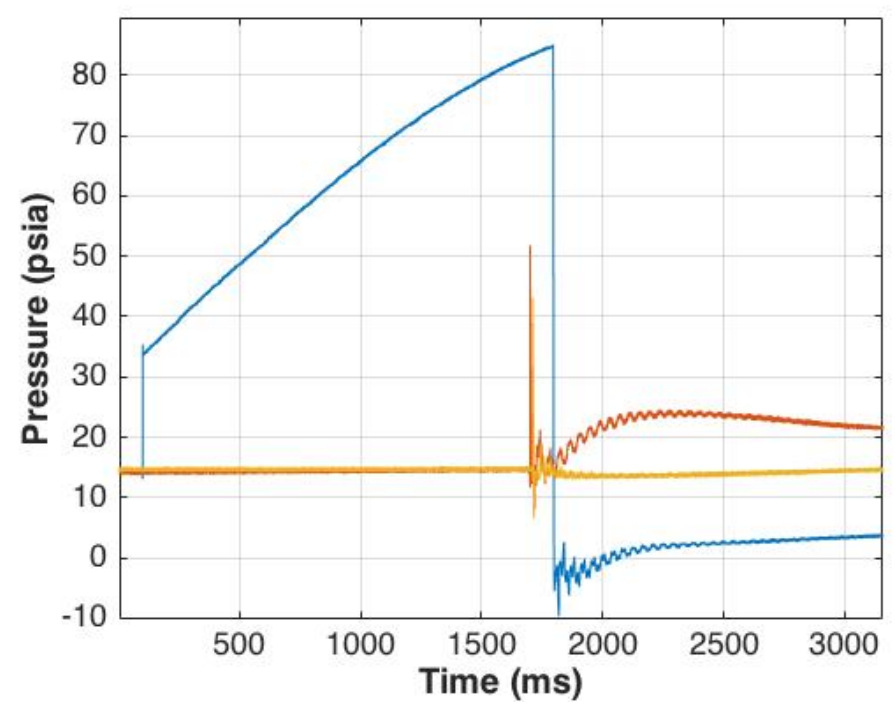

Figure 5.12: Time History of Incident Pressure Measurements

\subsection{Conclusions}

The results show that a compressed air shock tube has been successfully manufactured and instrumented following the design requirements set forth. The characterization of the shock tube also proved to be successful showing that the shock tube does indeed produce a shock wave where the experimental values do fall within an appropriate error range once a corrected conversion factor is used on the voltage data collected. In order to improve this set up a couple suggestions will be mentioned.

In the incident pressure measurements it is necessary to find a way to ensure the sensors are as flushed as possible with the inner surface perhaps the use of an endoscope will aid with this. Also as seen in the overall time history it is necessary to dampen the effect of the diaphragm bursting especially with those sensors with close proximity to the diaphragm connections as these feel the effect the most. Only so much can be done as ultimately how much the sensors are 
affected by the vibrations is a function of their sensitivity. Also for the purpose of obtaining better velocity measurements and more pressure data as the shock propagates down the tube it would be necessary to add more sensors to the set up which means obtaining a signal conditioner with more ports. This will also allow for incident pressure measurements to be made alongside total pressure measurements.

To improve the measurements for the test conducted at the exit of the tube the sensor fixture support arm must be positioned in such a way that it does not interfere with downstream measurements. But ultimately to fully understand what is going on in this region it is necessary to do a method of flow visualization so that all the flow features developed by this shock tube can be observed and appropriately aligned with their pressure measurement. Since this shock tube is used outside it would be best to do Schlieren photography using the sunlight as the light source and the second sensor on the driven tube as a time of arrival sensor to synchronize the data acquisition of the high speed camera.

Lastly any improvements to be made to the blast waves produced by the shock tube will need to take into consideration the needs of any future experiment and will require matching the data to that provided by ConWep [14]. In general stronger blast waves (or shock waves for that matter) could be produced by either changing the driver gas to one that is lighter such as helium or using a driver tube that has a larger diameter while the driven tube stays the same size. Additionally if a more consistent wave speed is required then a longer driven tube will be needed whereas if the jet effect at the exit (which accompanies any compressed gas wave) is to be reduced a shorter driven tube will be required. 


\section{REFERENCES}

[1] Mark, H., "The Interaction of a Reflected Shock Wave with the Boundary Layer in a Shock Tube," 1958.

[2] Dewey, J. M., Experimental Methods of Shock Wave Research, Springer, 2016.

[3] Arakeri, J. H., Krothapalli, D. D. A., and Lourenco, L., "Vortex ring formation at the open end of a shock tube: A particle image velocimetry study," Physics of Fluids, Vol. 16, 2004.

[4] Erfani, R., Zare-Behtash, H., and Kontis, K., "Influence of shock wave propagation on dielectric barrier discharge plasma actuator performance," Journal of Physics D: Applied Physics, Vol. 45, No. 22, 2012, pp. 225201.

[5] Roorkee, I., "Strength of Materials: Lecture 15," 2009.

[6] Bradley, J. N., Shock Waves in Chemistry and Physics, John Wiley and Sons Inc, 1962.

[7] Machinery's Handbook, Industrial Press Inc, 27th ed., 2004.

[8] "Application Design Patterns: Producer/Consumer," .

[9] Nishida, M., Handbook of Shockwaves Volume 1, chap. 4.1, Academic Press, 2001. 
[10] Davis, H. J. and Curchack, H. D., "Shock Tube Techniques and Instrumentation," 1969.

[11] Brun, R., "Shock Tube Design and Experiments," 2009.

[12] Needham, C., Blast Waves, Springer, 2010.

[13] Tasissa, A. F., Hautefeuille, M., Fitek, J. H., and Radovitzky, R. A., "On the formation of Friedlander waves in a compressed-gas-driven shock tube," 2015.

[14] Shridharani1, J. K., GarrettW.Wood1, Panzer1, M. B., Capehart, B. P., Nyein, M. K., A.Radovitzky, R., , and Bass, C. R. D., "Porcine Head Response to Blast," 2012.

[15] Dora, C. L., Saravanan, D., Karunakar, K., and Das, D., "Characteristics of Embedded-Shock-Free Compressible Vortex Rings: A Detailed Study Using PIV," Advances in Mechanical Engineering, 2011.

[16] Courtney, A. C., Andrusiv, L. P., and Courtney, M. W., "Oxy-acetylene driven laboratory scale shock tubes for studying blast wave effects," 2010.

[17] of the Aerodynamics Division N.P.L, D. W. H., "A Note on Shock Tubes," 1953.

[18] Budynas, R. G. and Nisbett, J. K., Shigley's Mechanical Engineering Design, McGraw-Hill, 9th ed., 2011.

[19] Parker O-Ring Handbook ORD 5700, Parker Hannifin Corporation, 2007. 
APPENDICES 
Appendix A

Sample Calculations 


\section{A.1 Shock Tube Thickness Calculation}

The calculation below shows how the minimum thickness was determined for shock tube walls once steel was chosen as the material.

$$
\begin{aligned}
& t_{\text {min }}=\frac{p d}{2 \sigma_{H}} \\
& t_{\text {min }}=\frac{120 p s i g * 2 i n}{2 * 30000 p s i} \\
& t_{\text {min }}=0.004 \mathrm{in}
\end{aligned}
$$


A.2 Number of Bolts 
So to select the number of bolts it is first necessary to determine $N_{\text {flange }}$

$$
\begin{aligned}
& N_{\text {flange }}=p_{4} * A_{p} \\
& N_{\text {flange }}=120 \mathrm{psig} * 3.14 \mathrm{in}^{2} \\
& N_{\text {flange }}=345.4 l b_{f}
\end{aligned}
$$

Now we calculate the force the bolt produces $N_{b o l t}=\frac{T}{c * M_{D}}$

$$
\begin{aligned}
& N_{\text {bolt }}=\frac{14 l b s-f t}{0.025 * 2 i n\left(\frac{1 f t}{12 i n}\right)} \\
& N_{\text {bolt }}=3360 l b_{f}
\end{aligned}
$$

Now we use the following equation to determine the number of bolts

$$
\begin{aligned}
& n=\frac{N_{\text {flange }}}{N_{\text {bolt }}} \\
& n=\frac{345.3 l b_{f}}{3360 l b_{f}} \\
& n=0.102 \operatorname{bolt}(s)
\end{aligned}
$$

Obviously you cannot have a part of a bolt so this number is rounded up:

$$
n=1 \operatorname{bolt}(s)
$$


A.3 Selecting Voltage Range 
This part begins by running the MATLAB function to solve for the expected shock strength value in Appendix C. Since the diaphragm is expected to burst at 84 psia our shock tube pressure ratio is 5.72 which leads our shock strength value to be 3.24 , which $p_{2}$ will be the following:

$$
\begin{aligned}
& p_{2}=\frac{p_{2}}{p_{1}} * p_{a m b} \\
& p_{2}=3.24 * 14.7 p s i a \\
& p_{2}=47.6 \text { psia }
\end{aligned}
$$

Now the sensors measure gauge pressure so the expected value for the sensors to measure $\left(p_{m}\right)$ is:

$$
\begin{aligned}
& p_{m}=p_{2}-p_{a m b} \\
& p_{m}=47.6 p s i a-14.7 p s i a \\
& p_{m}=32.9 p s i g
\end{aligned}
$$

Lastly we use the conversion factor $(\mathrm{CF})$ to convert measured pressure $\left(p_{m}\right)$ to measured voltage $\left(v_{m}\right): v_{m}=\frac{p_{m}}{C F}$

$$
\begin{aligned}
& v_{m}=\frac{32.9 p s i g}{295 \frac{p s i}{V}} \\
& v_{m}=0.12 \mathrm{~V}
\end{aligned}
$$

Now we know an expected value for the measured voltage so now we set the DAQ for a range that is close to this value. Note, we will also have to consider the bursting pressure of the diaphragm before setting the range. 
A.4 Determining Thickness of Mylar Diaphragm

$$
\begin{aligned}
p_{4}=\frac{2 t \sigma_{u l t}}{D} \\
p_{4}=\frac{2 * 0.005 i n * 28000 p s i}{2 i n} \\
p_{4}=140 p s i a
\end{aligned}
$$


Appendix B

PCB Adapter Drawing 


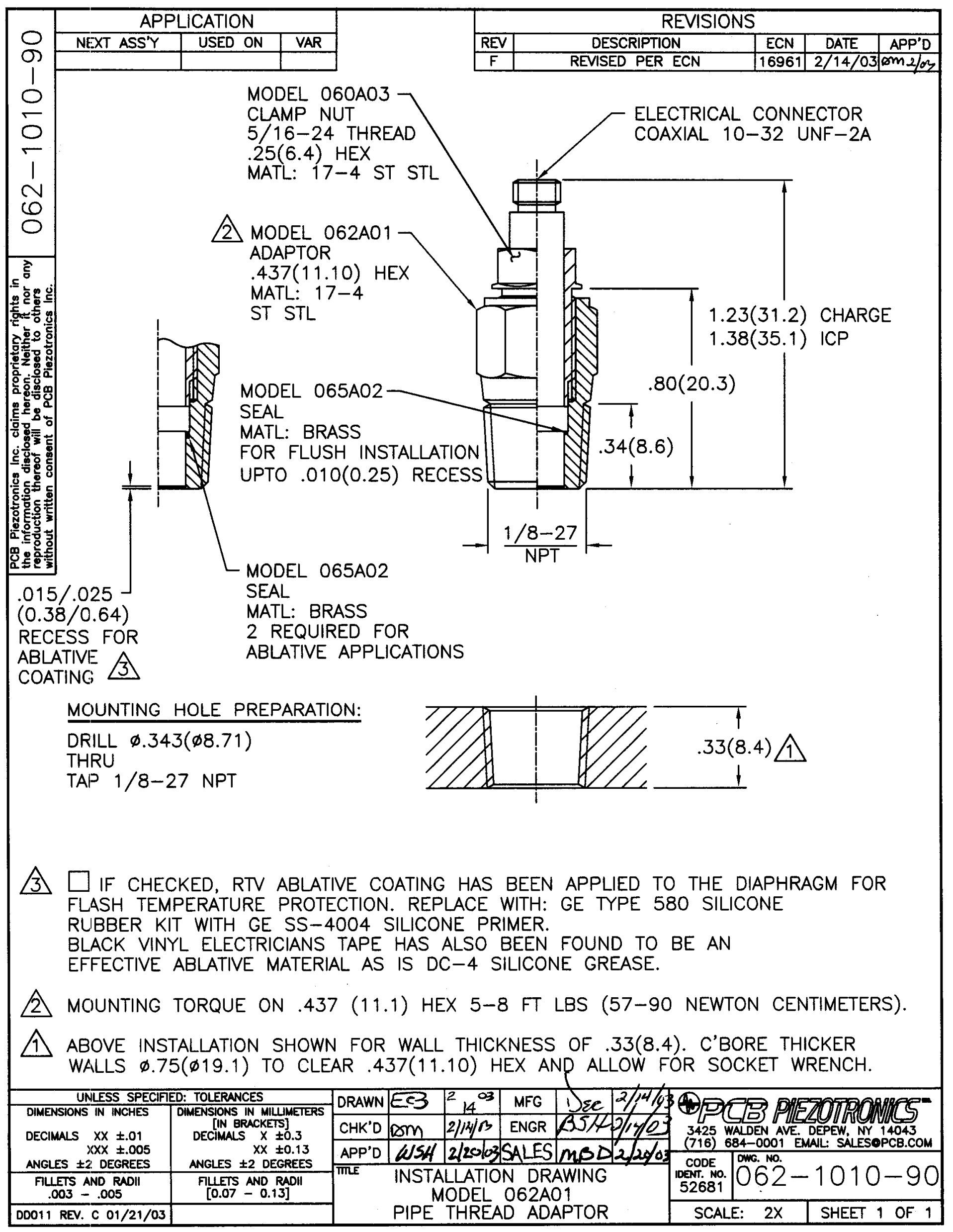


Appendix C

Testing Documents 
C.1 Safe Working Procedure 


\section{Cal Poly Open End Shock Tube SWP}

This is a working document on the safe working procedure for operating the shock tube. It is broken up into 4 parts:

- Pre-Start

- Emergency Shutdown

- Operating Procedures

- Breakdown

In order to assure safe operation it is necessary to read through the whole document before being to run the shock tube this will ensure the users are fully aware and prepared to run experiments.

\section{$\underline{\text { Pre-Start }}$}

This section is to be done before starting anything to ensure a safe working and testing environment with as many controlled variables as possible.

1. Before proceeding to the area it is necessary that there are at least two users present under no circumstance is this shock tube to be operated alone.

2. When entering the area ensure all users have the following PPE:

a. Closed Toed Shoes and Pants

b. Safety Glasses

c. Hearing Protection

3. With a minimum with 2 users present proceed to the outer yard of the propulsion lab where the blast wall is located.

4. Once here position the test stand to line up with the bolts holes on the floor and proceed to bolt down the test stand. Be sure to physically and visually check that the test stand is fully secured to the floor (See Figure 1)

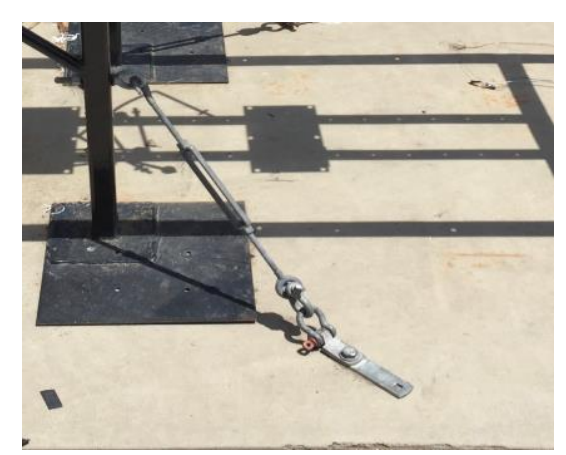

Figure 1: Test stand secured to the floor 
5. Now proceed to join the driver and the driven section the shock tube together (See Figure 3 for a diaphragm of these steps).

a. Before putting the two sections together it is necessary to check the all connections (see Figure 2) are properly secure and not loose. Also check for any cracks on the gasket.

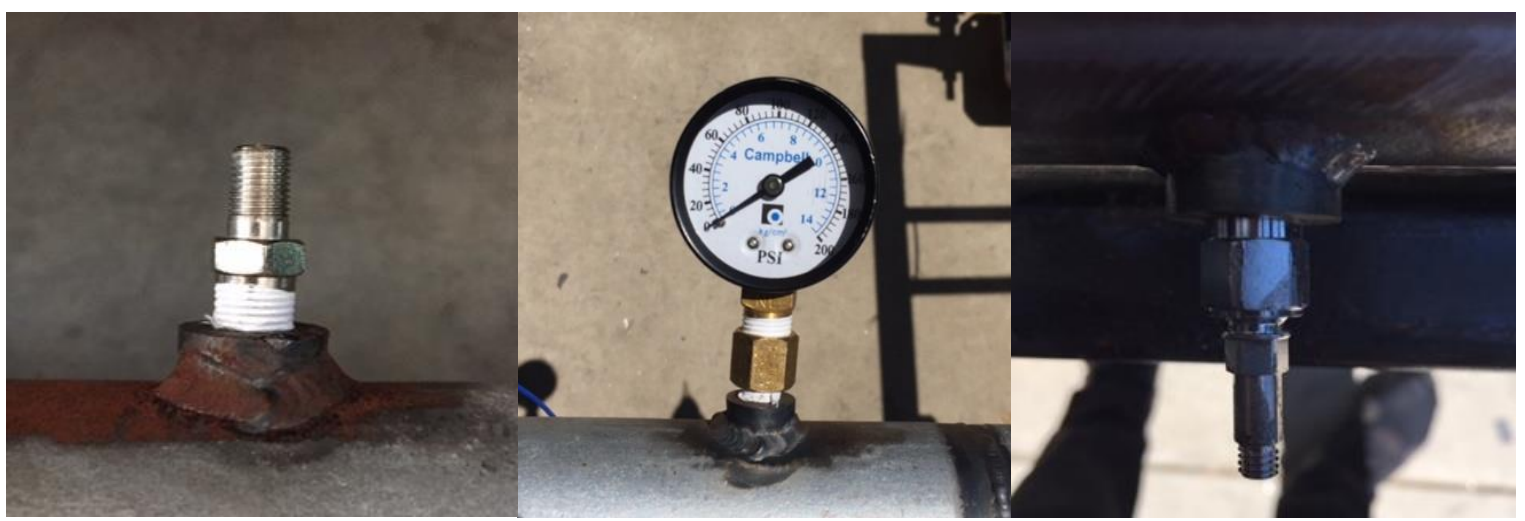

Figure 2: Connections properly installed on the shock tube.

b. Insert bolts on the driver section flange with the washers.

c. Next on the inner side of the flange apply the rubber sealant then proceed to put on the gasket and feed the bolts through the holes on the gasket.

d. Now place the Mylar diaphragm and feed the bolts through the precut holes.

e. Put the washers on the outer side and proceed to completely tighten nut to ensure a secure seal between the two flanges in the pattern shown in Figure 3.
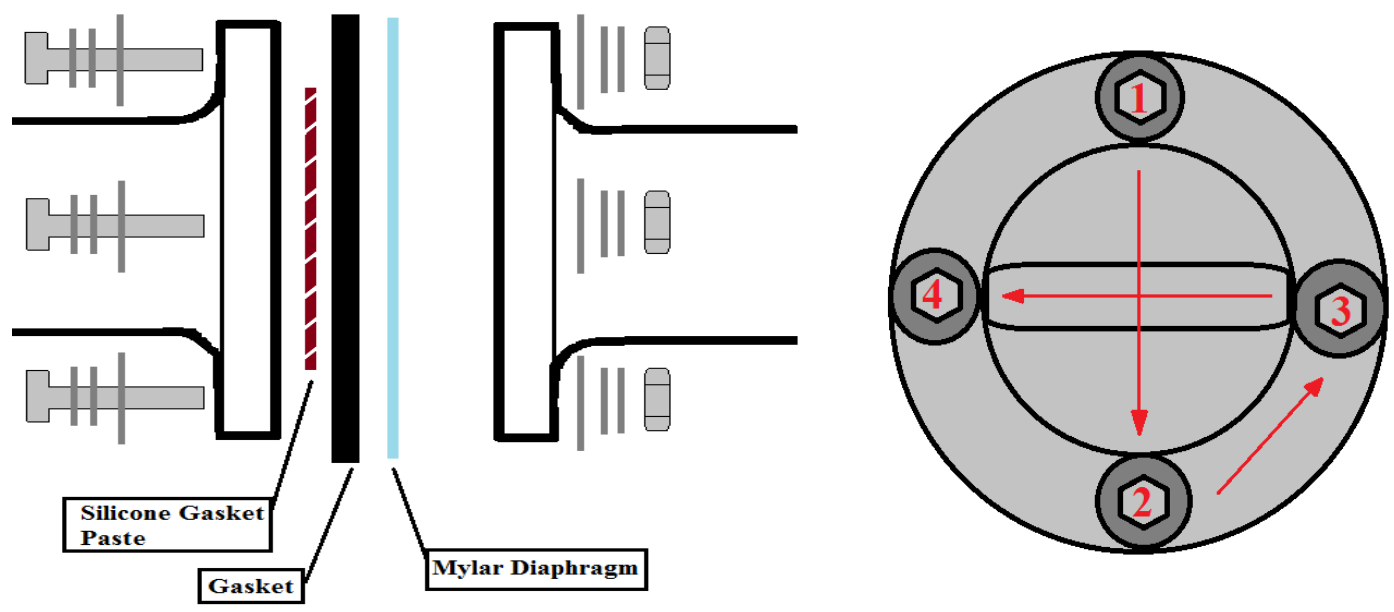

Figure 3: How to properly seal the tube. (Left) Order of feeding hardware (Right) Bolt tightening pattern. 
6. Once the two parts are fully secure proceed to place the tube on the test stand. Be sure that the open side is facing the blast wall.

7. At this point place the rubber vibration dampers on the tube and secure the tube to the test stand using the hose clamps and the bolt clamps. Be sure to visually and physically inspect that the tube is fully fastened to the test stand (See Figure 4).

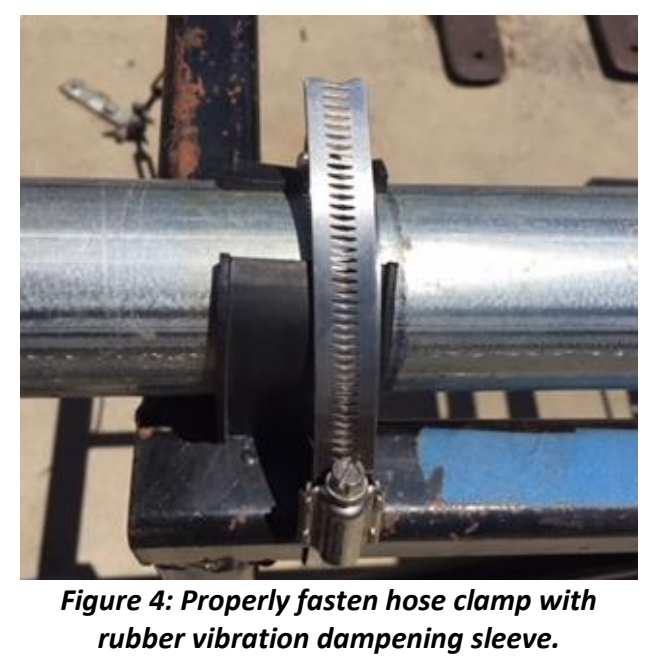

8. Now inspect and prepare the area for testing.

a. Check for any loose debris or move any obstacles away from the open side of the tube.

b. Proceed to close all doors

c. Brief all users of test plan and emergency procedures.

9. You are now ready to commence operation of shock tube.

\section{Emergency Shutdown Procedures}

There are a few scenarios in which the shock tube operation will have to be shut down immediately and users will have to determine what the issue is and amending it before proceeding to test.

1. If during pressuring of the tube to desired pressure ratio it is noticed that there is a leak at one of the connection immediately stop and through secondary valve release the pressure Attend to leaking valve and reseal before attempting to run again.

2. Premature breakage of the diaphragm may occur (expected breakage $~ 84$ psia for 0.003 " thick Mylar diaphragm), immediately stop filling with air and first check that there is no sign of damage to the flange connections. Now carefully take apart the flange assembly and proceed to remove all parts to then once again follow pre-start procedures to secure the connection between the two parts. 


\section{$\underline{\text { Operating Procedures }}$}

Now with the shock tube put together and secured on the rocket test stand this section will cover on how to conduct tests with the shock tube.

1. Never during the operation of the shock tube shall any person participating in the experiment stand in front or near the open end of the shock tube.

2. Before beginning to pressurize the shock turn on the LabView Shocktube VI and begin to run it.

3. Now proceed to inspect the air service hose and ensure the Schrader valve connector is properly connected with the hose (See Figure 5)

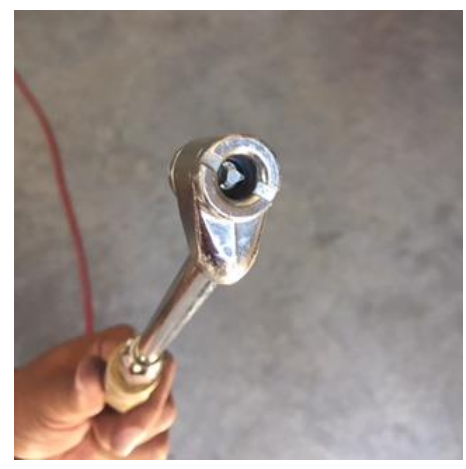

Figure 5: Schrader valve connector attached to air hose.

4. Turn the air service on then:

a. Proceed to begin filling the tube with air while consistently monitoring the pressure on the Bourdon gauge.

b. Begin data acquisition as soon as the pressure gauge begins to show increase in pressure and continue until the diaphragm bursts.

c. Once the diaphragm burst stop adding air to the driver tube.

5. Once the 'bang' of the diaphragm has been heard continue data collection for 45 seconds and visually inspect for any damage.

6. Wait until data acquisition is complete then proceed to prepare for next run.

\section{$\underline{\text { Breakdown }}$}

Now with all desired testing complete this section covers the breakdown of the experiment and proper storage procedures of the shock tube.

1. Turn off the VI and computer.

2. Put away all sensors and DAQ in box and rollaway to its position on the roll cart.

3. Now proceed to take apart the diaphragm assembly and properly disposing of the used diaphragms in any trash can in the lab. 
4. Now unstrap the driven section of the shock and remove from test stand and take to its position, following this unstrap the driver section and put away.

5. Unbolt test stand from ground and proceed to put away in the corner of the yard.

6. Put away all connectors and other supplies used during testing.

7. Turn off air service and bleed the air out of the system.

8. Once all things are put away do an area check to ensure nothing was left behind.

9. Take off PPE and put away in appropriate cabinets.

10. When leaving vicinity ensure all doors are properly closed. 
C.2 Test Procedure 


\section{Cal Poly Open End Shock Tube Testing Plan}

This document serves the purpose to layout the testing plan followed to acquire data to characterize the open end shock tube. There are four parts to this testing plan:

- $\quad$ Setting up the DAQ

- Incident/Velocity Data Acquisition

- Reflected/Blast Wave Data Acquisition

- Pressurizing the Driver Tube

Before following this testing plan it is important to follow the Safe Working Procedure (SWP) developed for this open end shock tube to ensure the safety of everyone operating the tube.

\section{Setting up the DAQ}

Follow these steps to set up the DAQ:

1. Before turning on the DAQ connect the signal conditioner and the 3 sensors using the BNC connectors as shown in Figure 1.

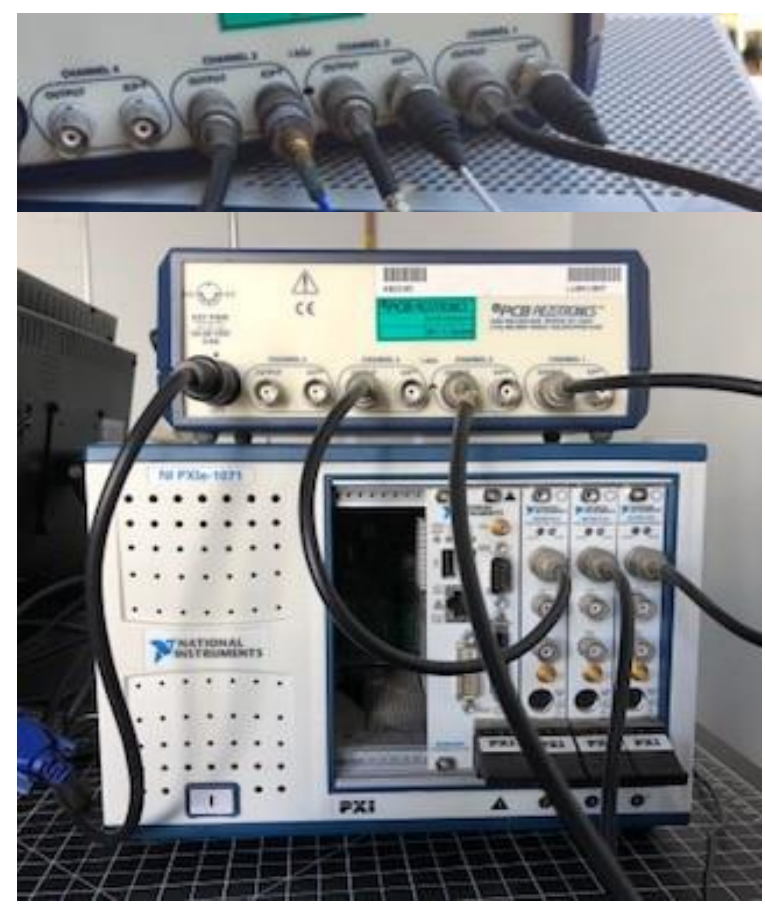

Figure 1: Connecting signal conditioner, sensors to DAQ

2. Turn on the DAQ and login the password is : shocktube1

3. Next turn on the signal conditioner and give 15-20 mins to warm up and settle. 
4. Open the latest version of the $\mathrm{VI}$ and set the appropriate settings. Figure 2 shows the user interface of the VI, Table 1 shows the settings used for data acquisition.

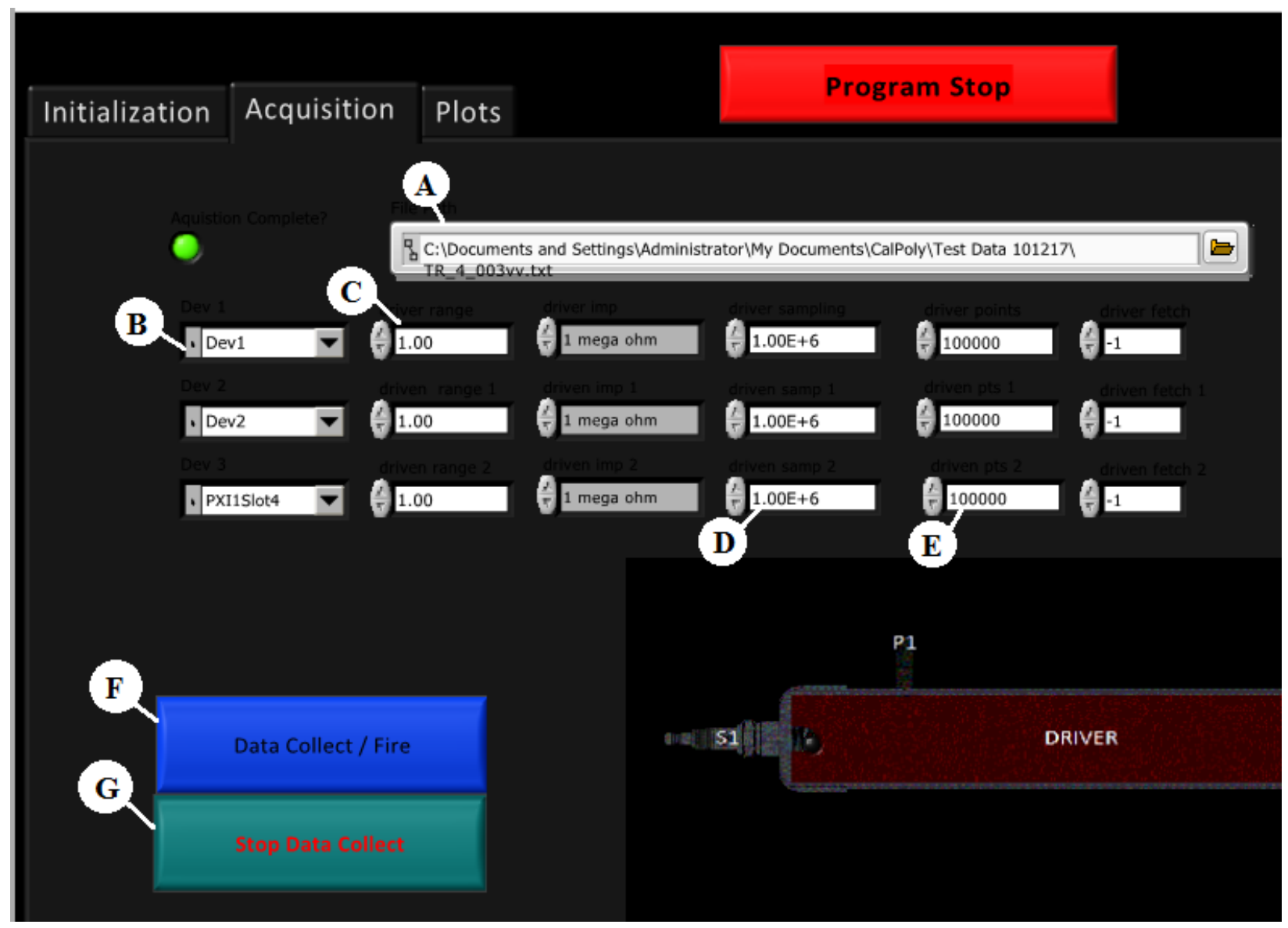

Figure 2: User Interface of DAQ VI

- Label $A$ is where the user sets the file path where the text file will be saved

- Label B sets up the digitizer card that the user wants to use for a specific channel

- Label $C$ sets the voltage (vertical) range

- Label D sets up the sampling frequency

- Label E sets up the fetch

- Label $\mathrm{F}$ is the button to press to start the writing the data to the text file

- Label $\mathrm{G}$ is the button to stop the VI from writing data to text file 


\begin{tabular}{|c|c|}
\hline Condition & Setting \\
\hline Sampling Frequency & $1 \mathrm{MHz}$ \\
\hline Sensor Range & $0.5 \mathrm{~V}$ \\
Sensor Fetch & 100000 points \\
\hline
\end{tabular}

Table 1: DAQ Settings

5. Now with all the settings correctly set up proceed to set up sensors in test of choice.

\section{Incident/Velocity Data Acquisition}

In this section the procedure for attaching the sensors to the shock tube are outlined.

1. Using the PCB adaptor diagram place all 3 ICP pressure sensors into the adaptors and tighten to the manufacturer's requirements.

2. Next, determine which sensor will be for the driver tube and which ones will go to the driven tube. It is a good idea to label them and to keep track of which DAQ card you connected them into as well.

3. Now proceed to connect the each one of the sensors into it specific slot on the shock tube as shown in Figure 3. Make sure to torque to manufacturer requirements as listed on the PCB adaptor diagram.

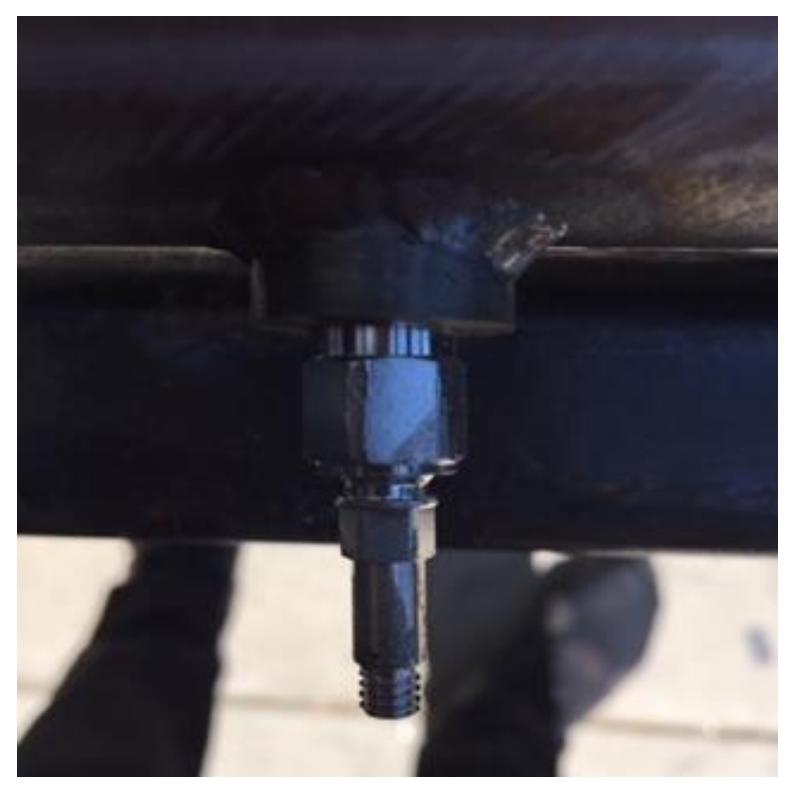

Figure 3: Attaching ICP Pressure Sensor to Shock Tube 
4. You can now go to the Pressurizing the Driver Tube section to conduct an experiment.

\section{$\underline{\text { Reflected/Blast Wave Data Acquisition }}$}

In this section the procedure for setting up for the sensors to measure the exit flow is outlined. Figure 4 shows how to place the sensors in positions $\mathrm{Al}, \mathrm{All}, \mathrm{BI}, \mathrm{BII}$.

1. If you did an Incident/Velocity test first then proceed to remove the sensors from the driven tube and leave the driver tube sensor attached. Otherwise if starting with no sensors attached, only put one sensor in the adaptor and follow the PCB adaptor diagram to properly install on specified slot with the manufacturer's required torque (see Figure 3).

2. The other two sensors place inside sensor fixture either in position (I) or (II).

3. Attaching sensor fixture to test arm and then proceed to place on stand (stool).

4. Place whole configuration now so that sensors are either in position $(A)$ or $(B)$.

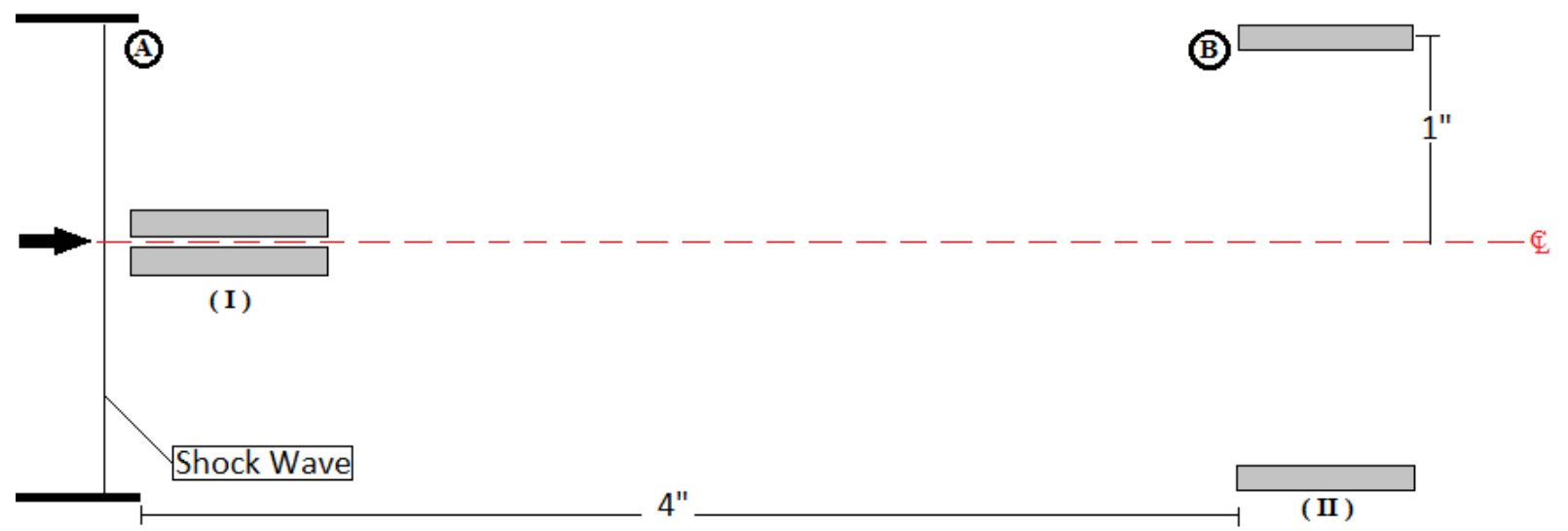

Figure 4: Sensor Configurations

5. Now secured down sensors by placing $100 \mathrm{lbs}$ of weights as shown in Figure 5. 


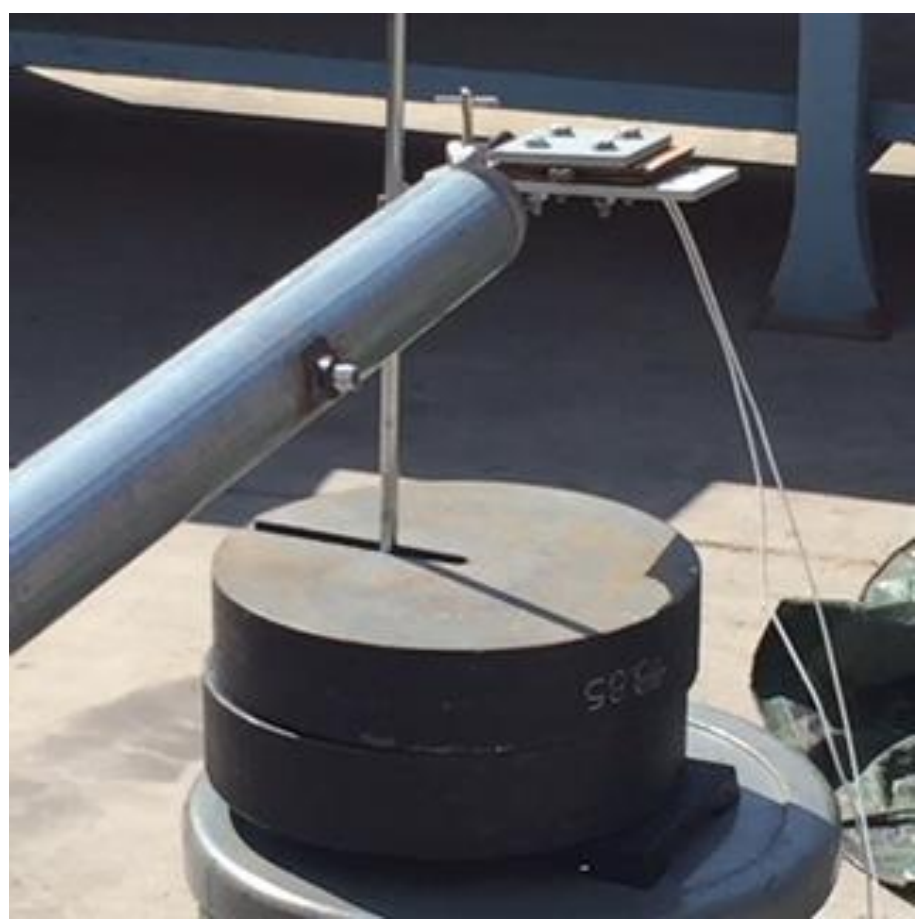

Figure 5: Pressure sensors secured with weights

6. Proceed to Pressurizing the Driver Tube section.

7. To move sensors into different position do so without the driver pressurized and removed weights and then move entire configuration then secure again by placing weights on again.

\section{Pressurizing the Driver Tube}

This section serves the purpose of outlining how to pressurize the driver tube and collecting data for a given test run. This section assumes you have fully read the SWP.

1. Follow steps to properly seal tube as shown in SWP.

2. With the Schrader valve connected to an air supply proceed to attached to inlet air valve.

3. As the tube is pressurizing on the VI pressure the button on Label F (See Figure 2) to begin to data collection.

4. Continue filling with air until the diaphragm burst is heard. While this happening you should see the Bourdon gauge rise up in pressure to 84 psia and then drop when the diaphragm has ruptured.

5. After $\mathbf{4 5}$ secs has passed after the diaphragm burst proceed to stop data acquisition by pressing the button under Label $\mathrm{G}$.

6. Follow these steps for any consecutive test run you wish to do. 
C.3 MATLAB Code for Post Processing 


\section{Table of Contents}

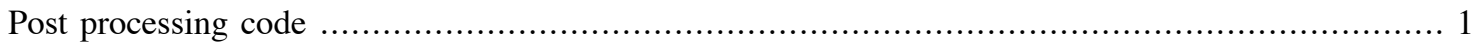

Calculate Mach shock number theory to compare to velocity .............................................. 5

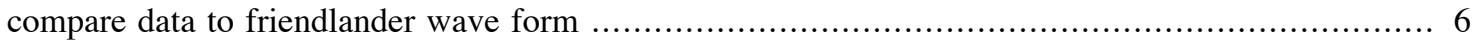

\section{Post processing code}

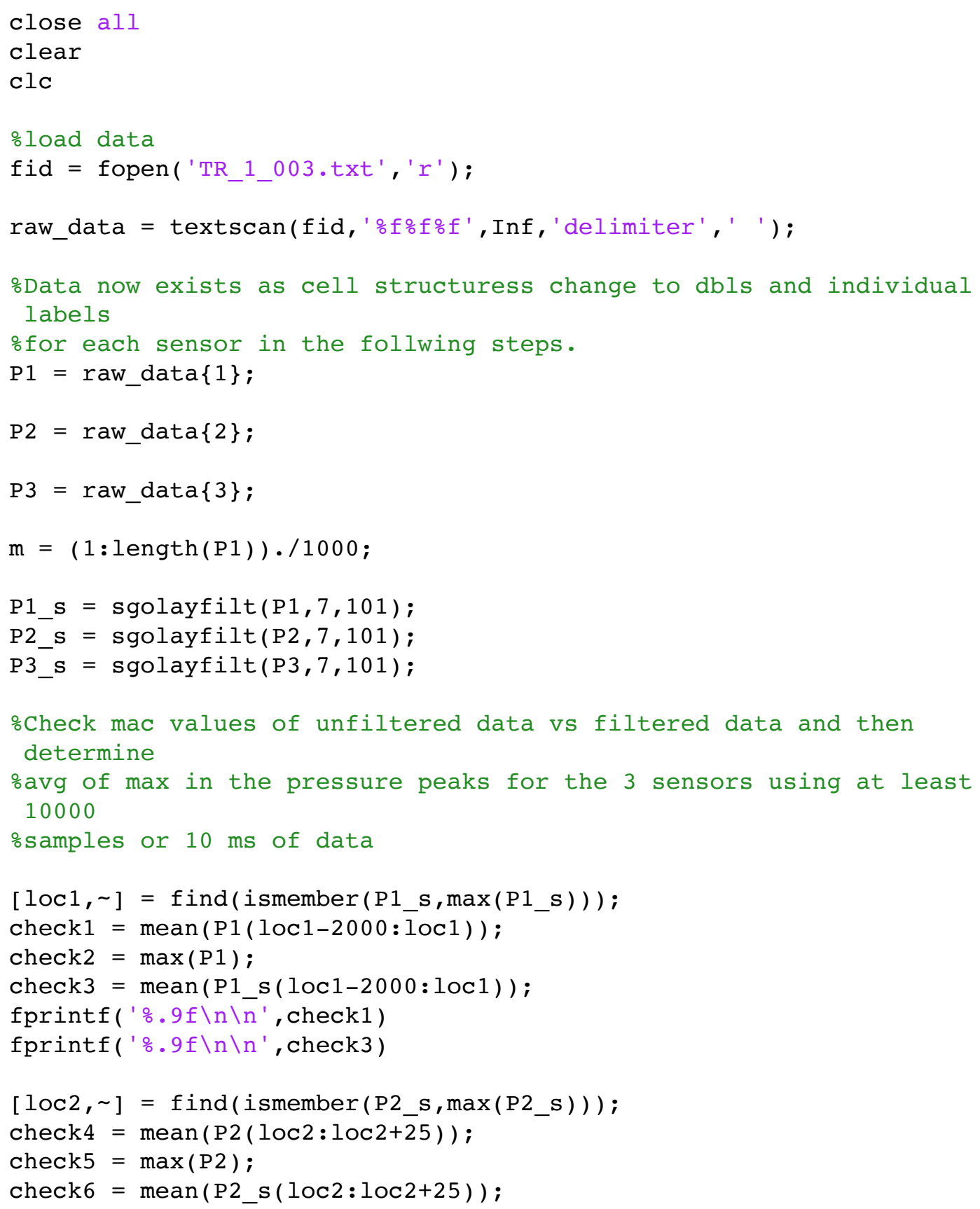




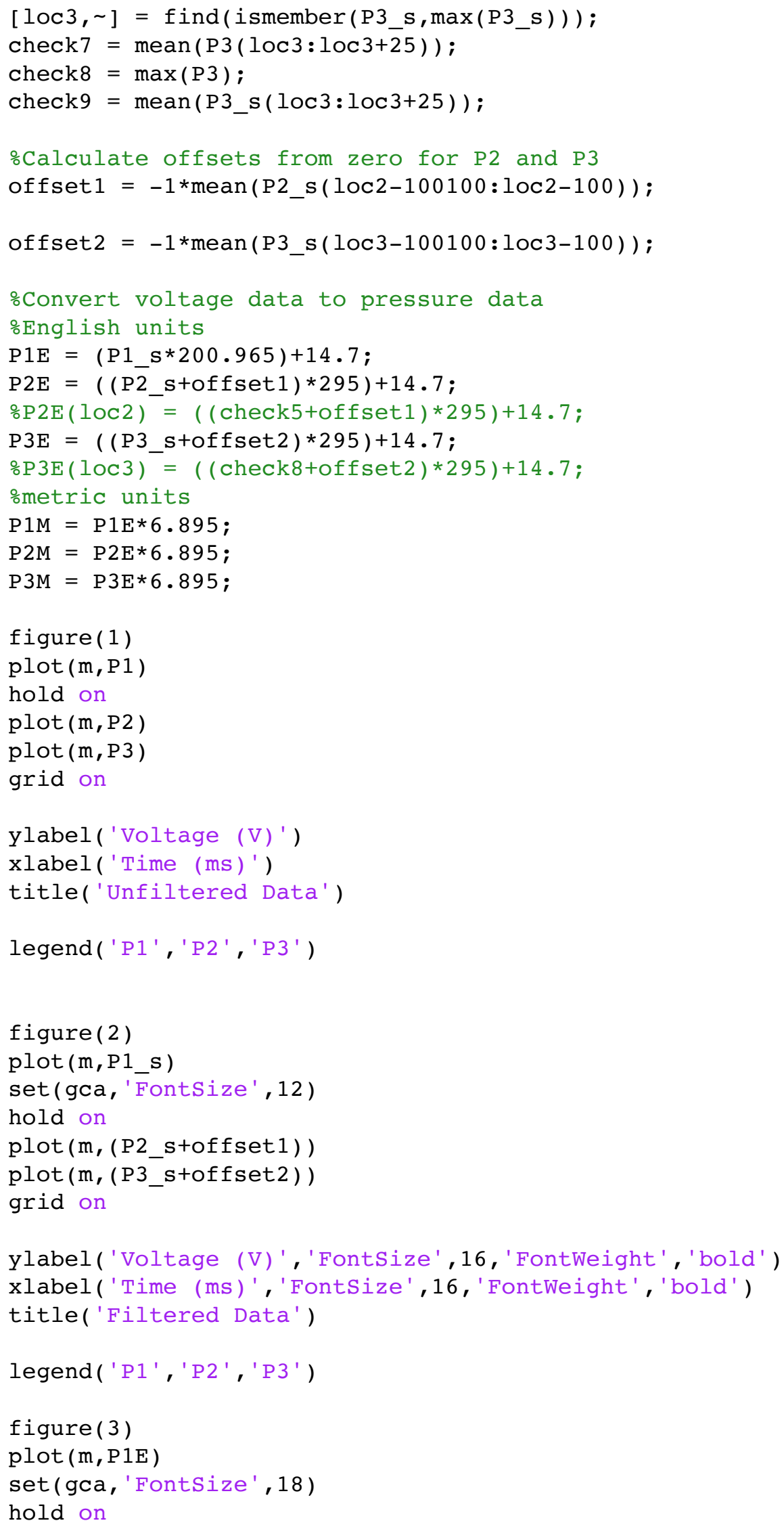




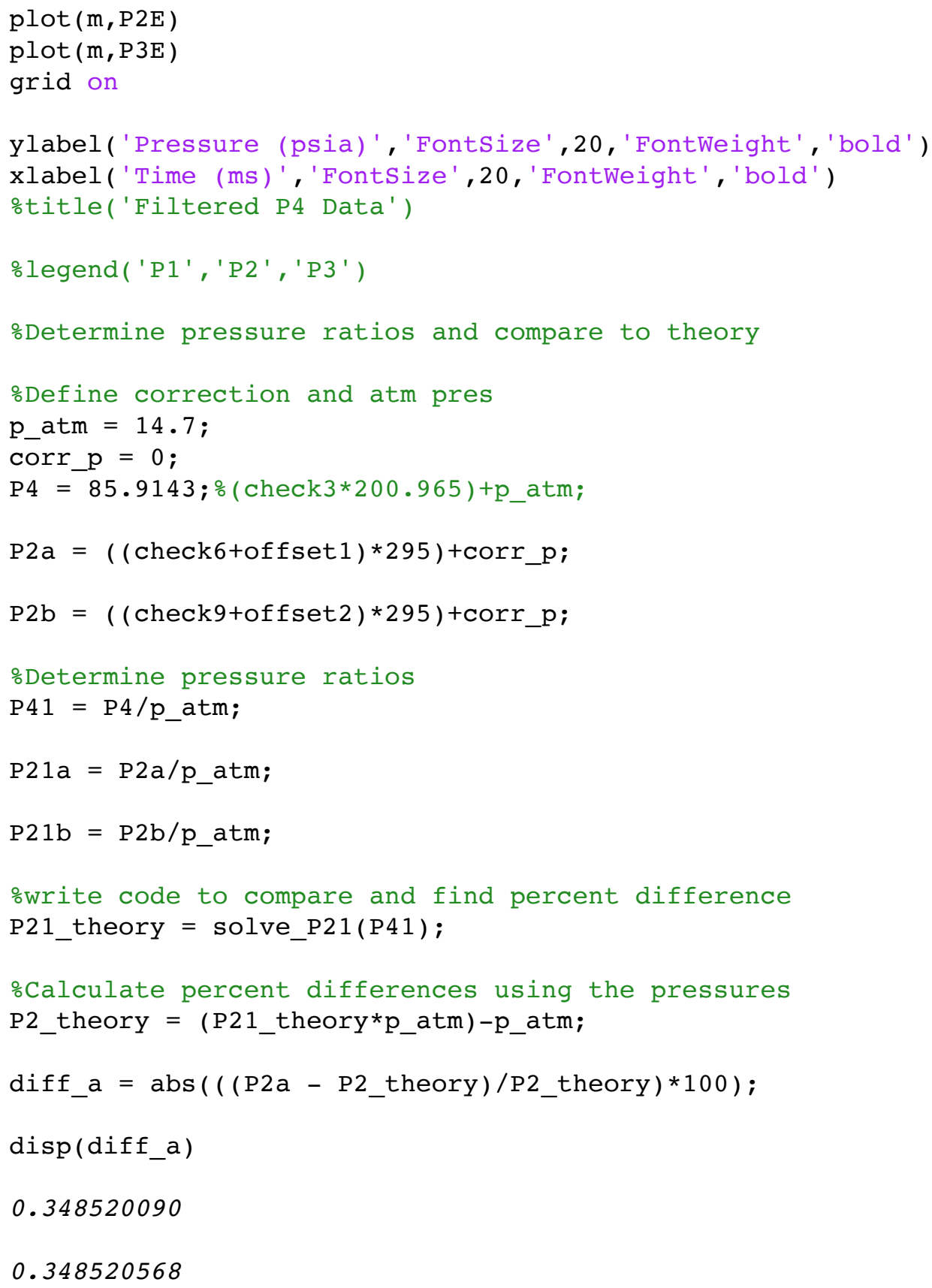




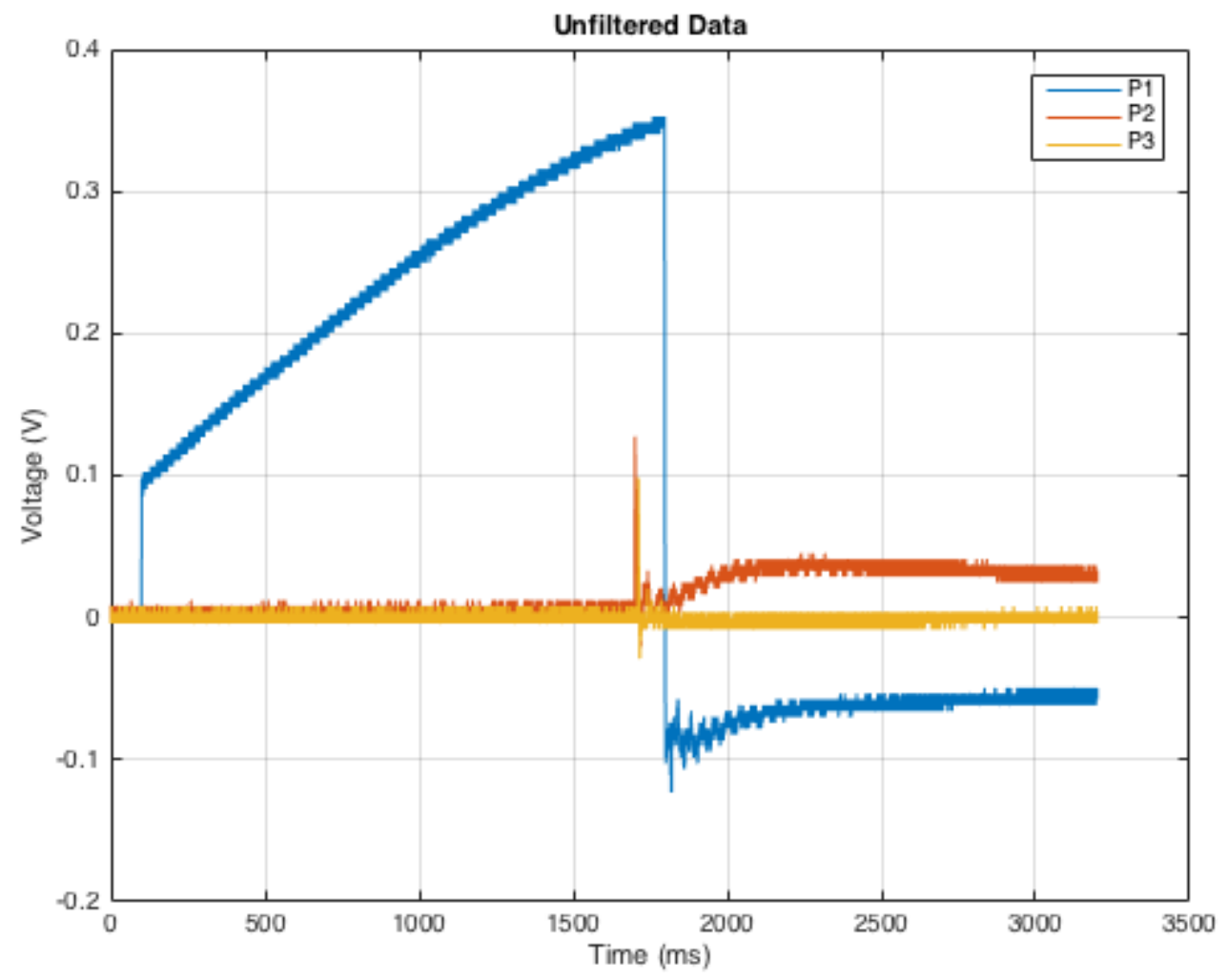

Filtered Data

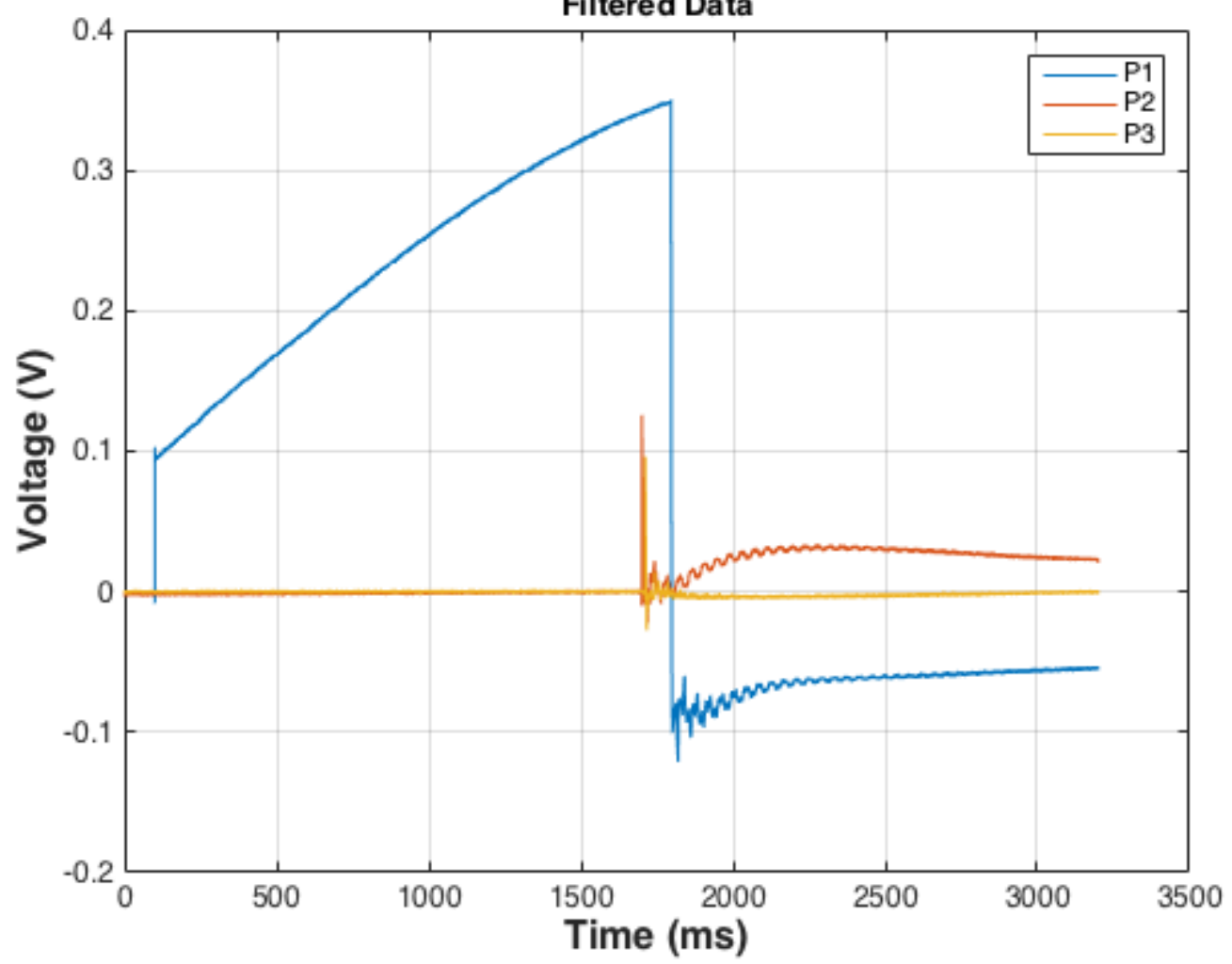




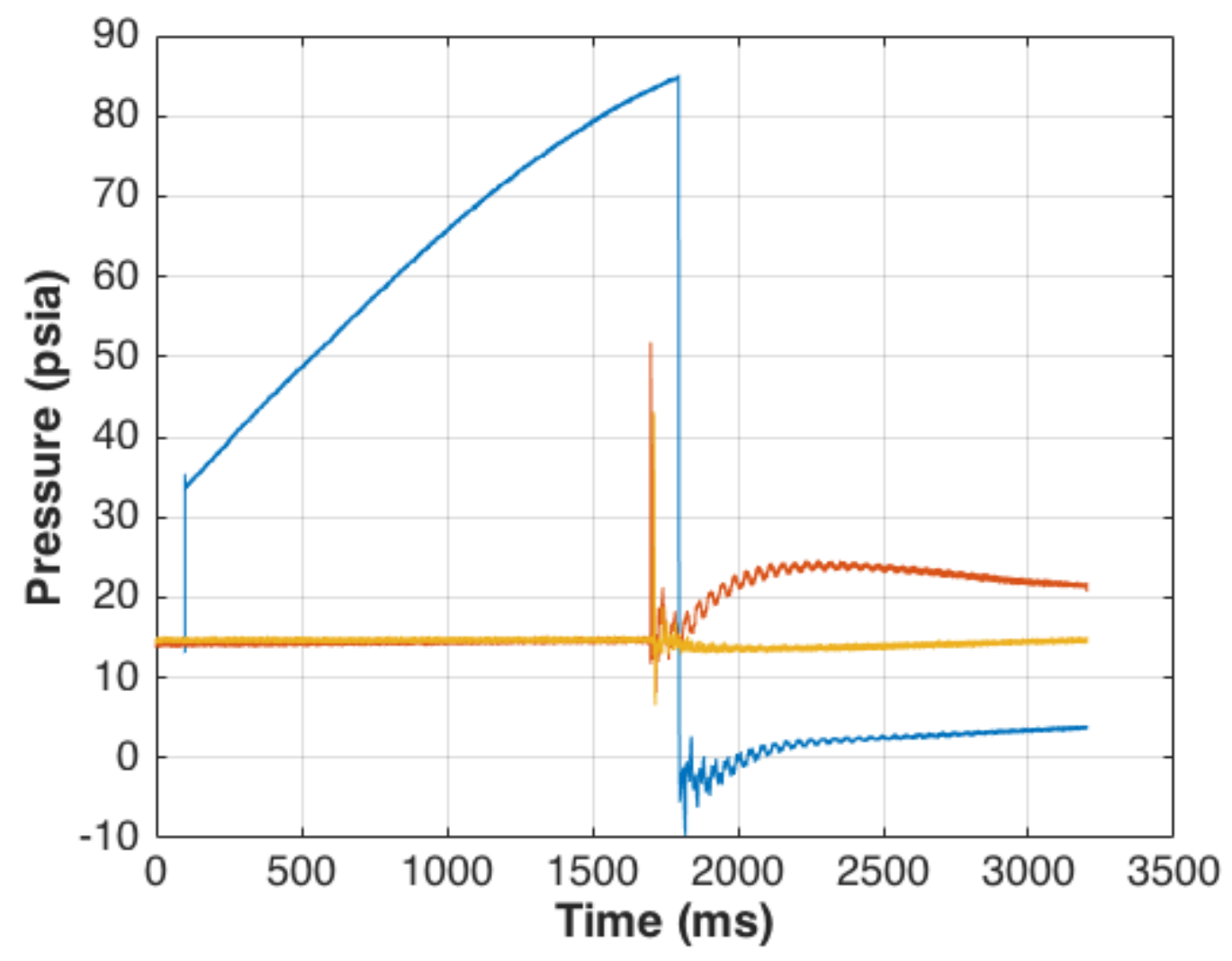

\section{Calculate Mach shock number theory to com- pare to velocity}

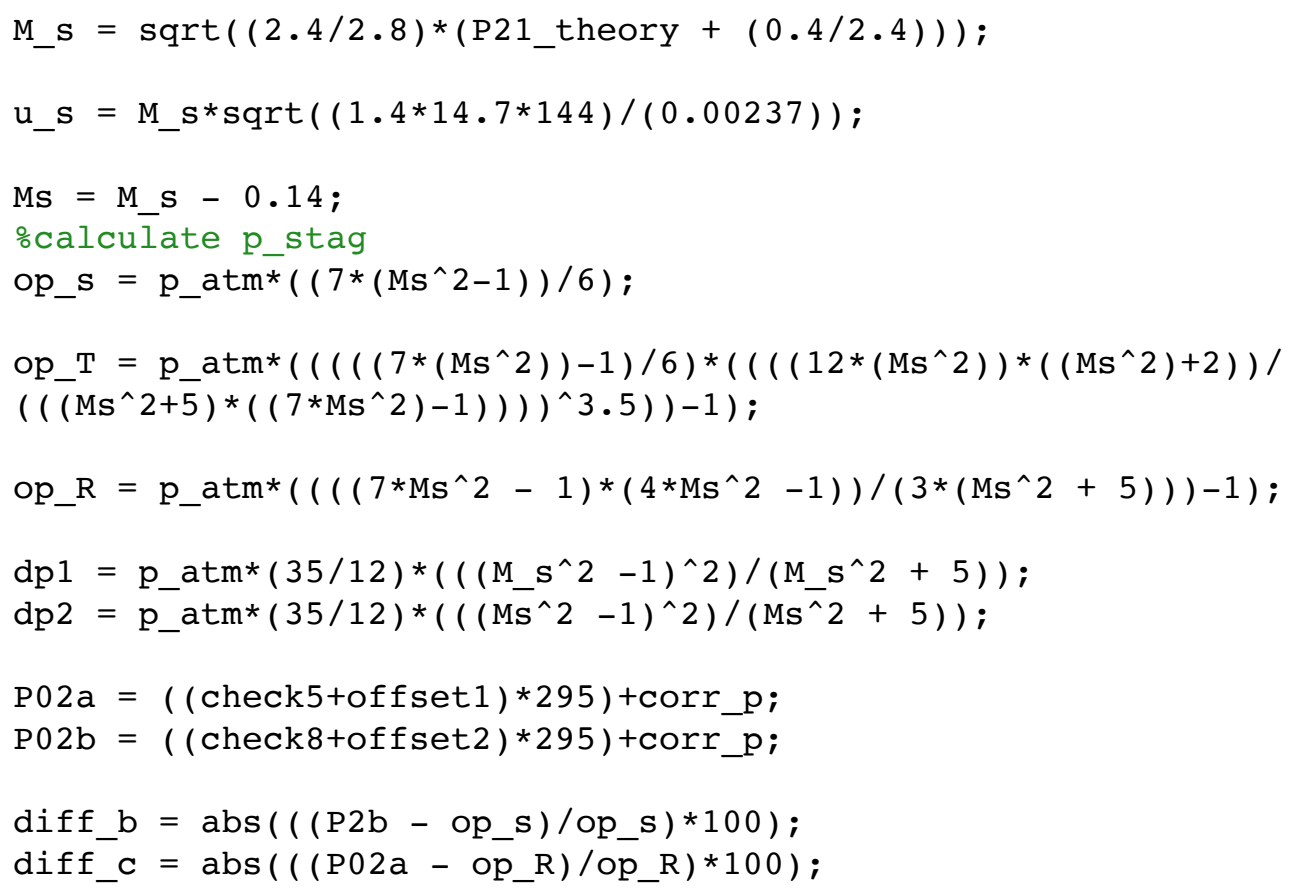




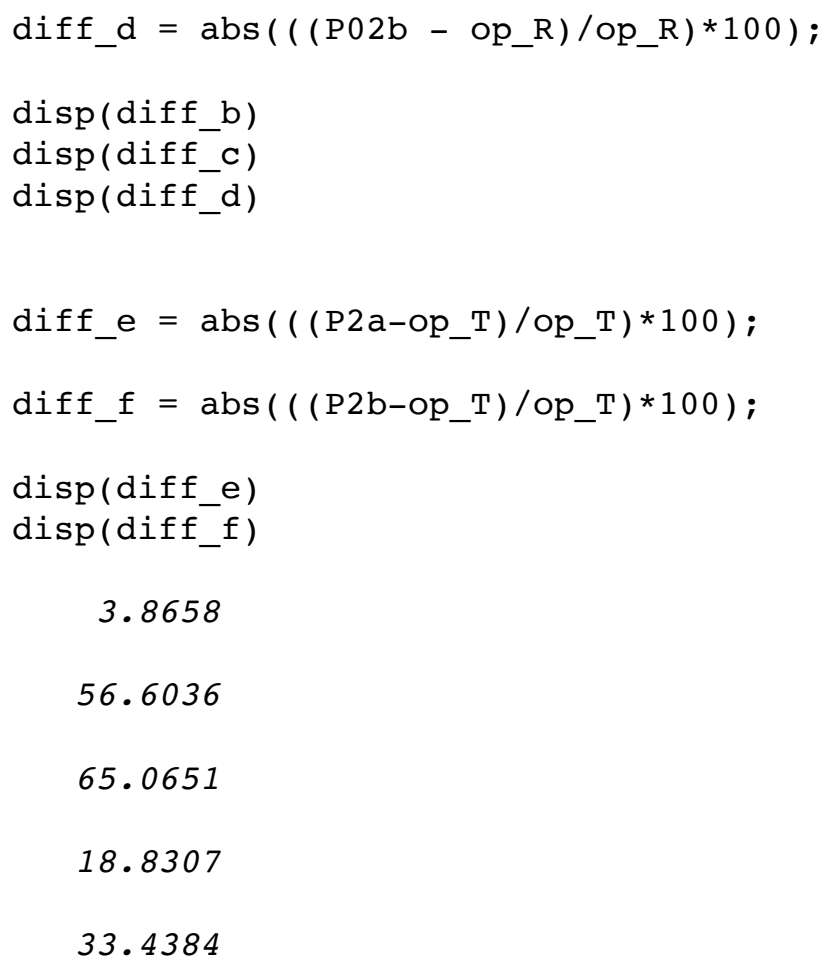

\section{compare data to friendlander wave form}

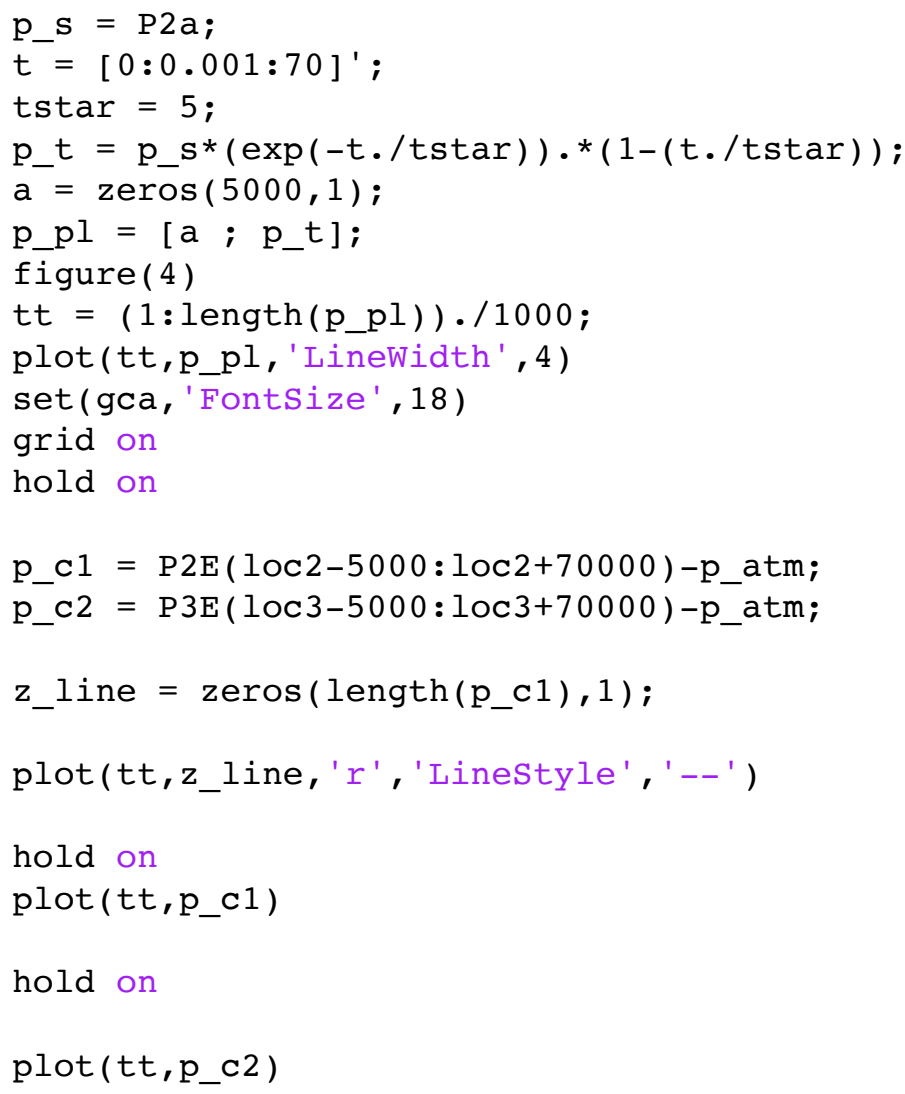


oset (gca, 'FontSize', 18)

ylabel('Pressure (psig)', 'FontSize', 20, 'FontWeight', 'bold')

xlabel ('Time (ms) ', 'FontSize',20, 'FontWeight', 'bold')

otitle('Comparison to Friedlander Equation')

legend('Theorectical Friedlander Wave','Zero / Atmosphere','Exp M1', 'Exp M2');

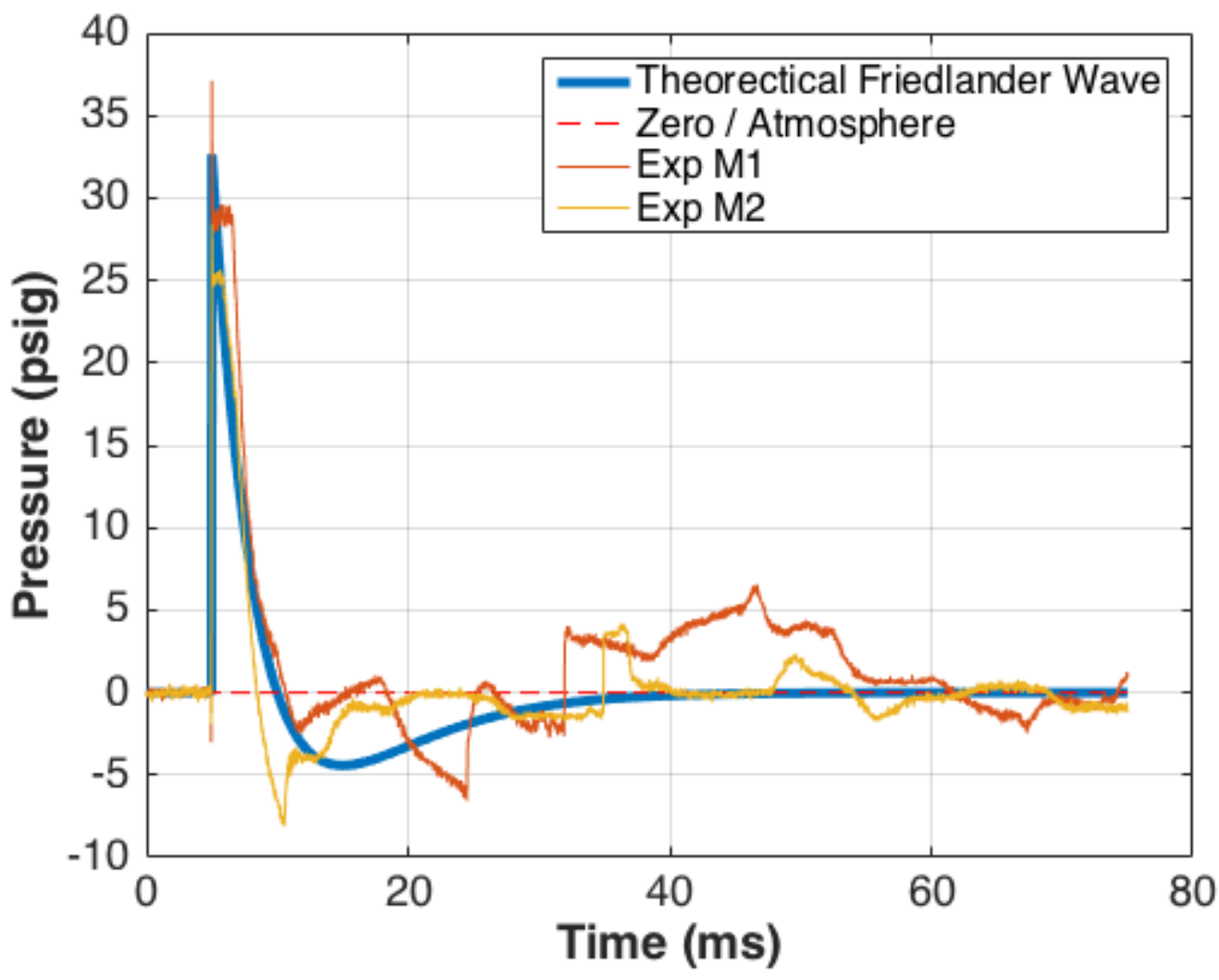

Published with MATLAB® R2015b 


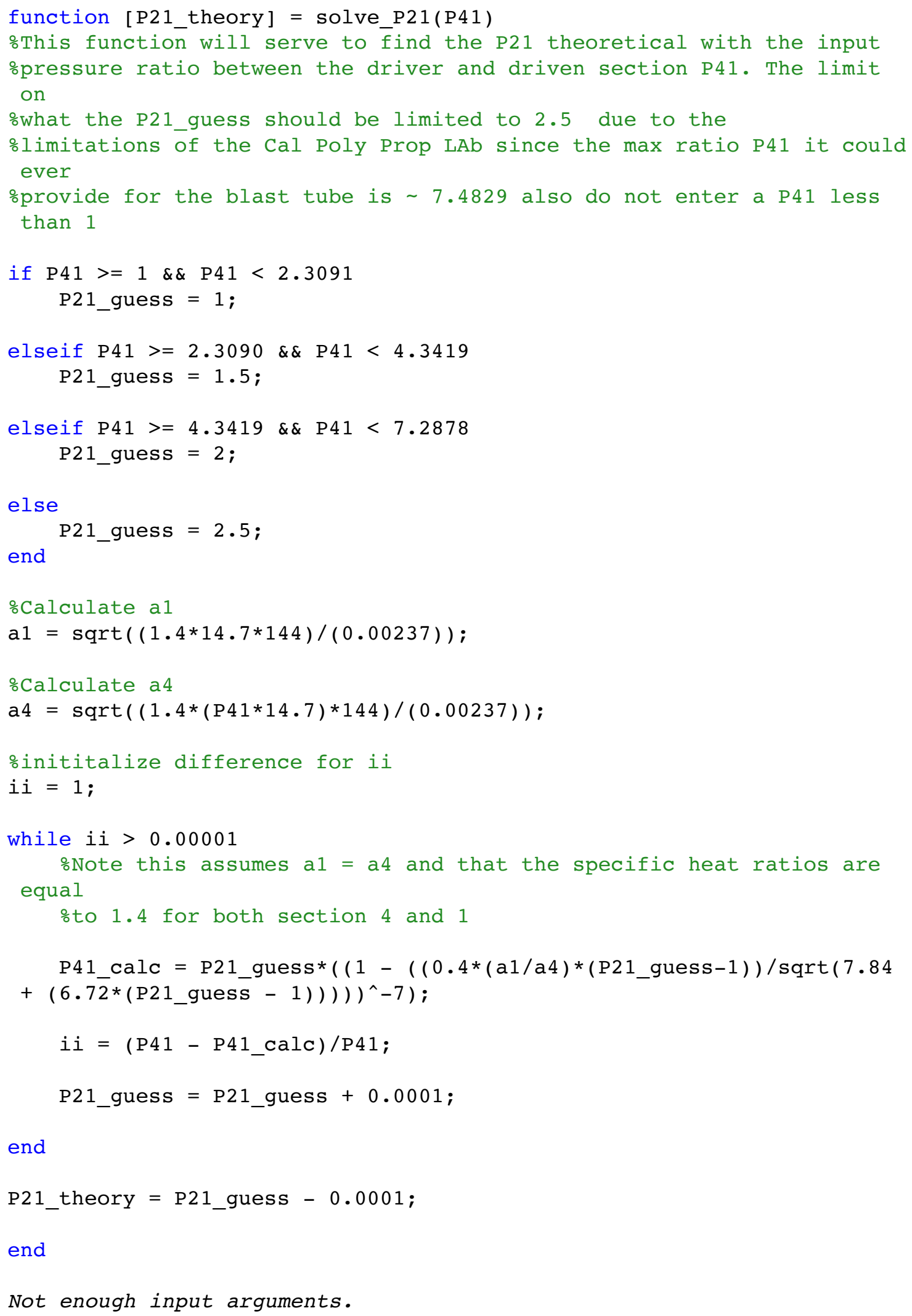




\section{Post processing code for shock velocity}

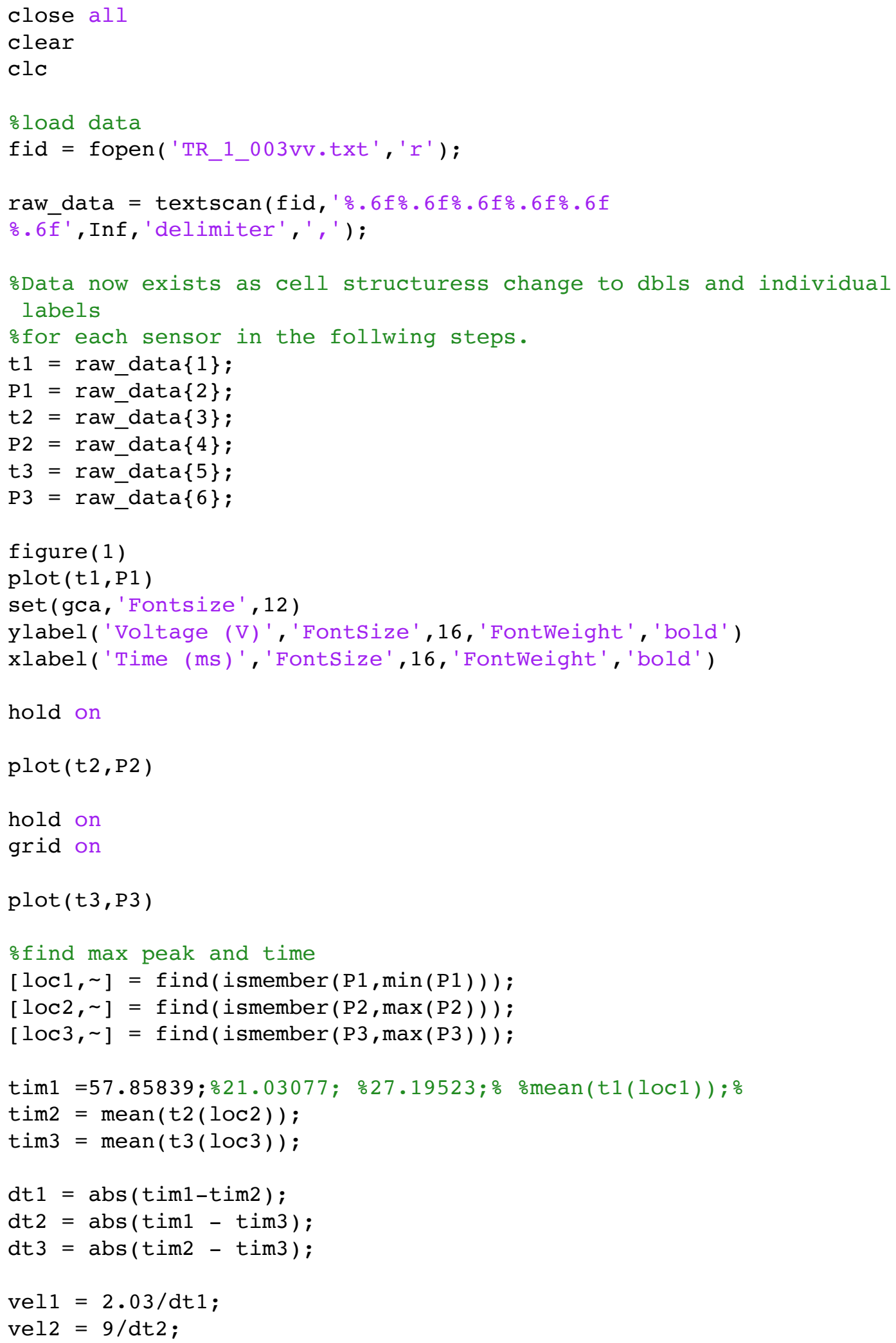


$\operatorname{vel3}=7 / \mathrm{dt} 3$;

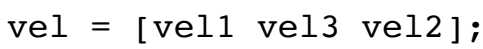

$\mathrm{n}=\left[\begin{array}{lll}1 & 2 & 3\end{array}\right]$;

figure (2)

$\operatorname{plot}(\mathrm{n}, \mathrm{vel})$

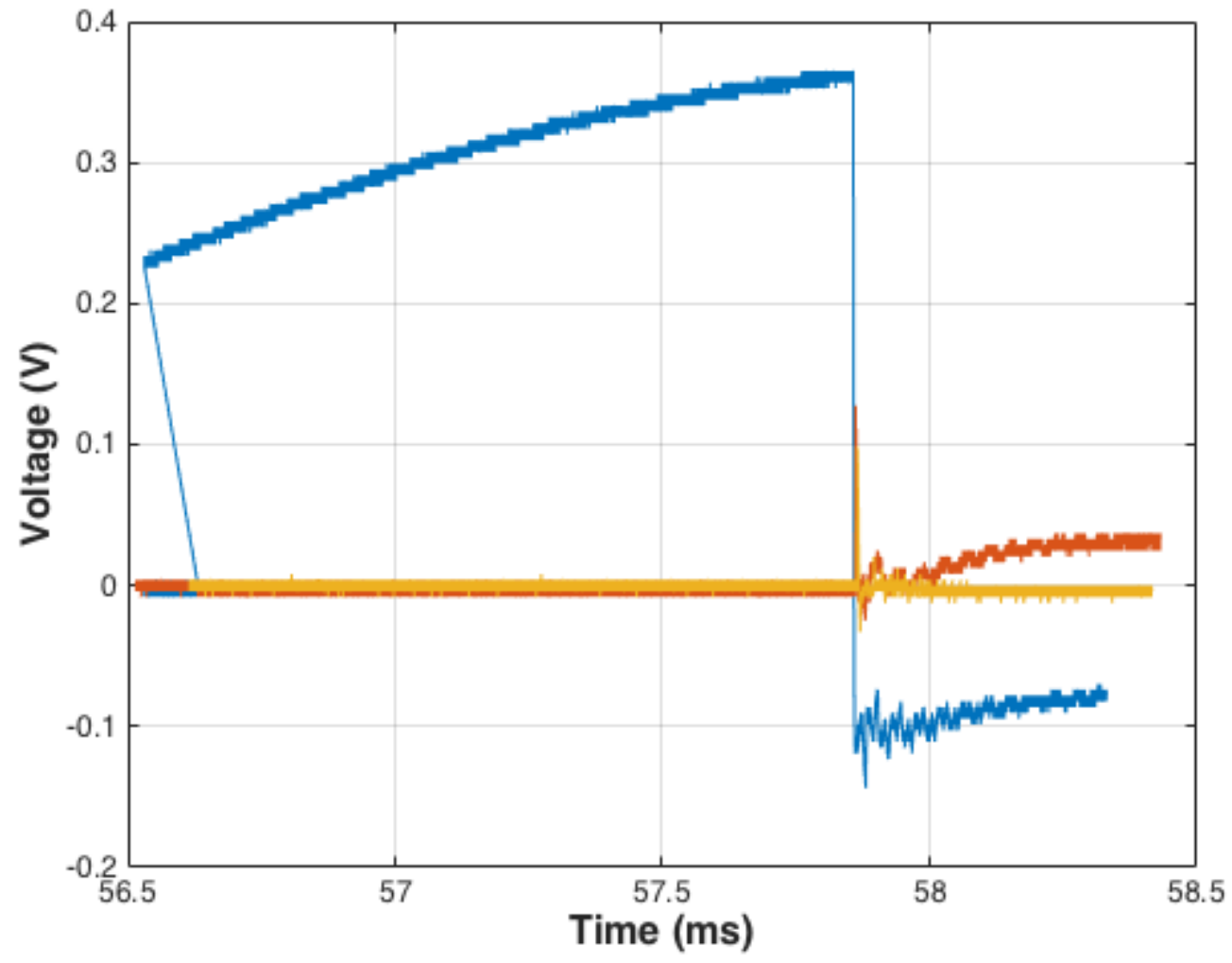




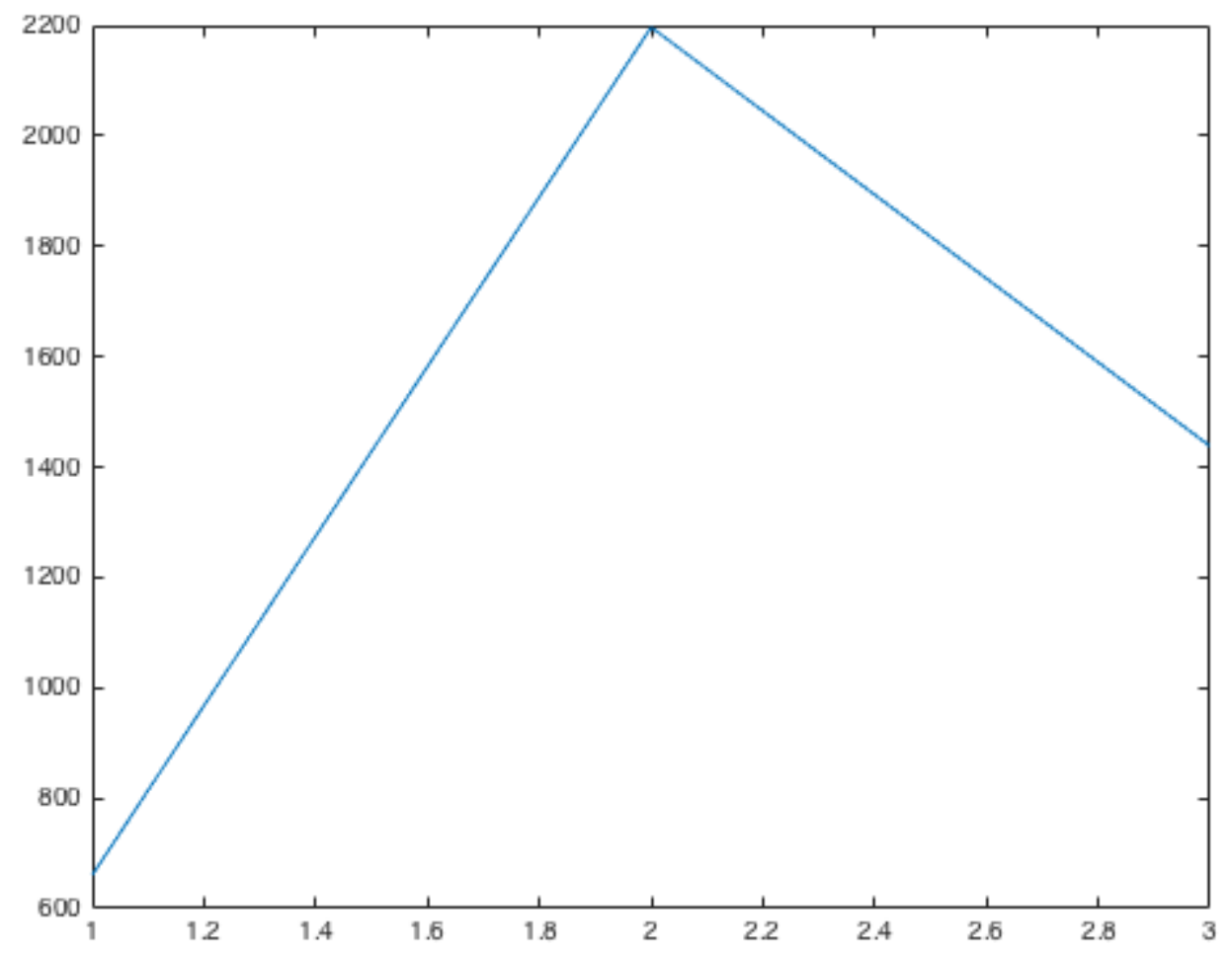

Published with MATLAB® R2015b 


\section{Some post processing calcs}

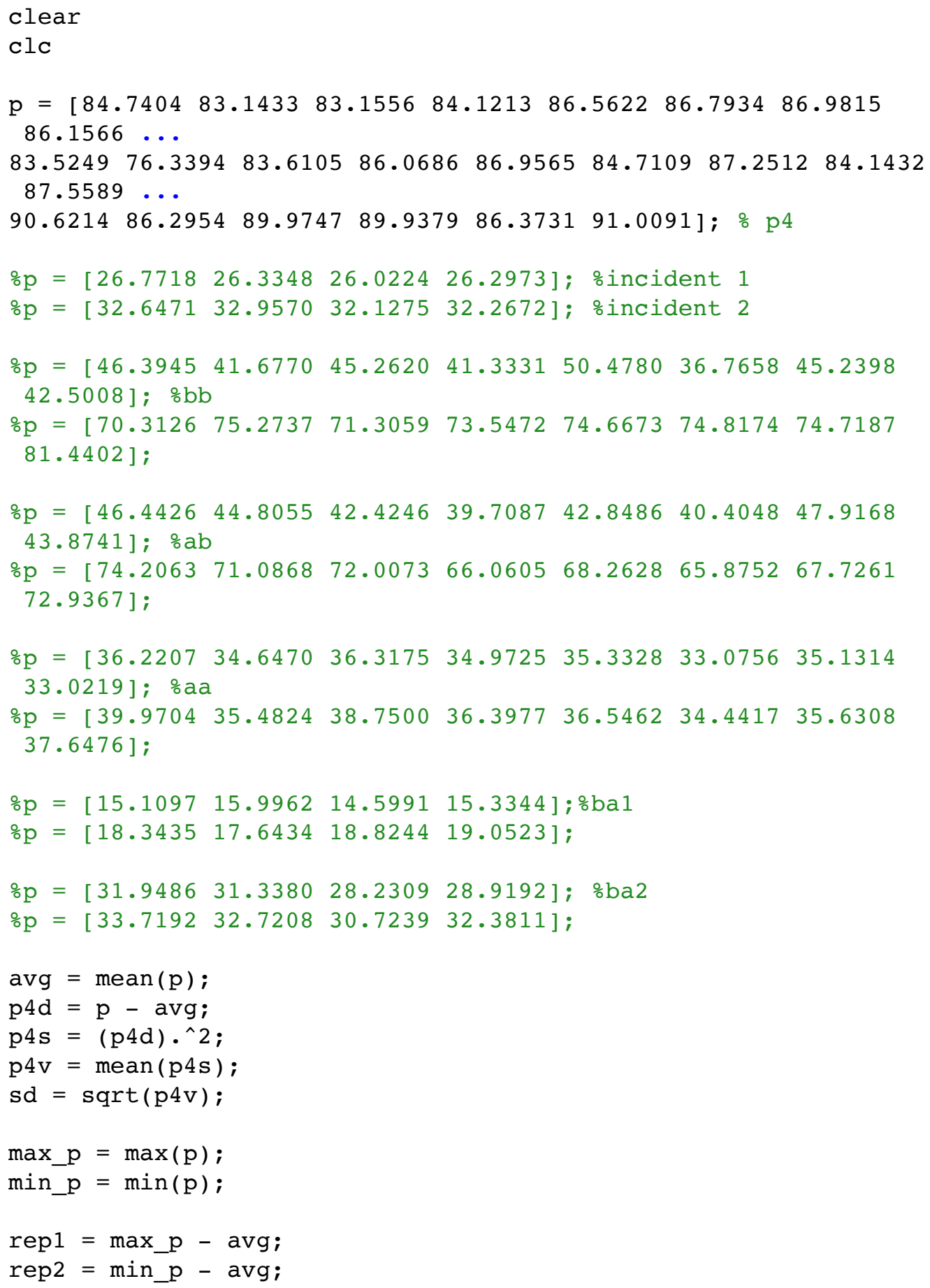

Published with MATLAB® R2015b 
Appendix D

Unfiltered Data 


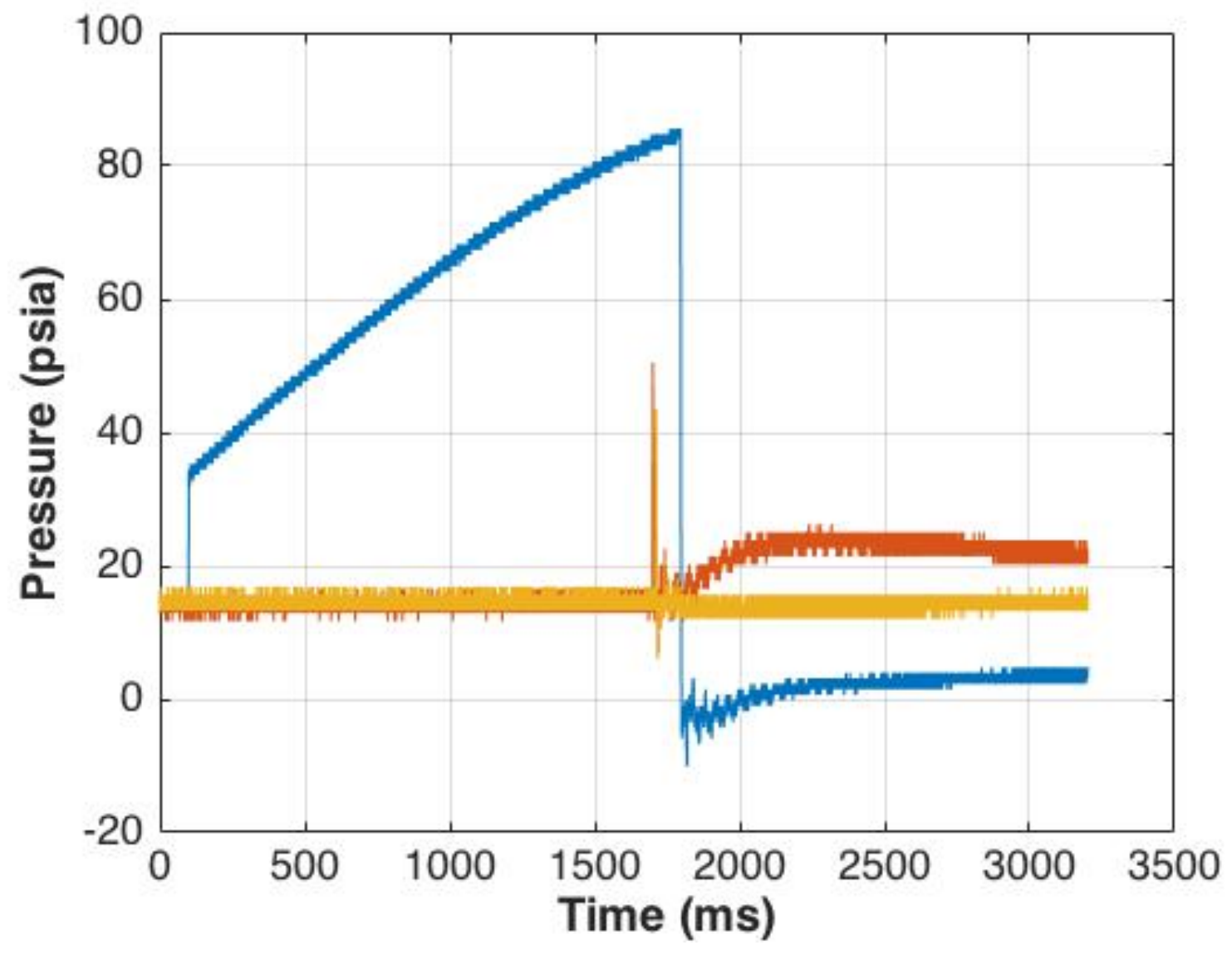

Figure D.1: Unfiltered Incident Pressure Measurements 


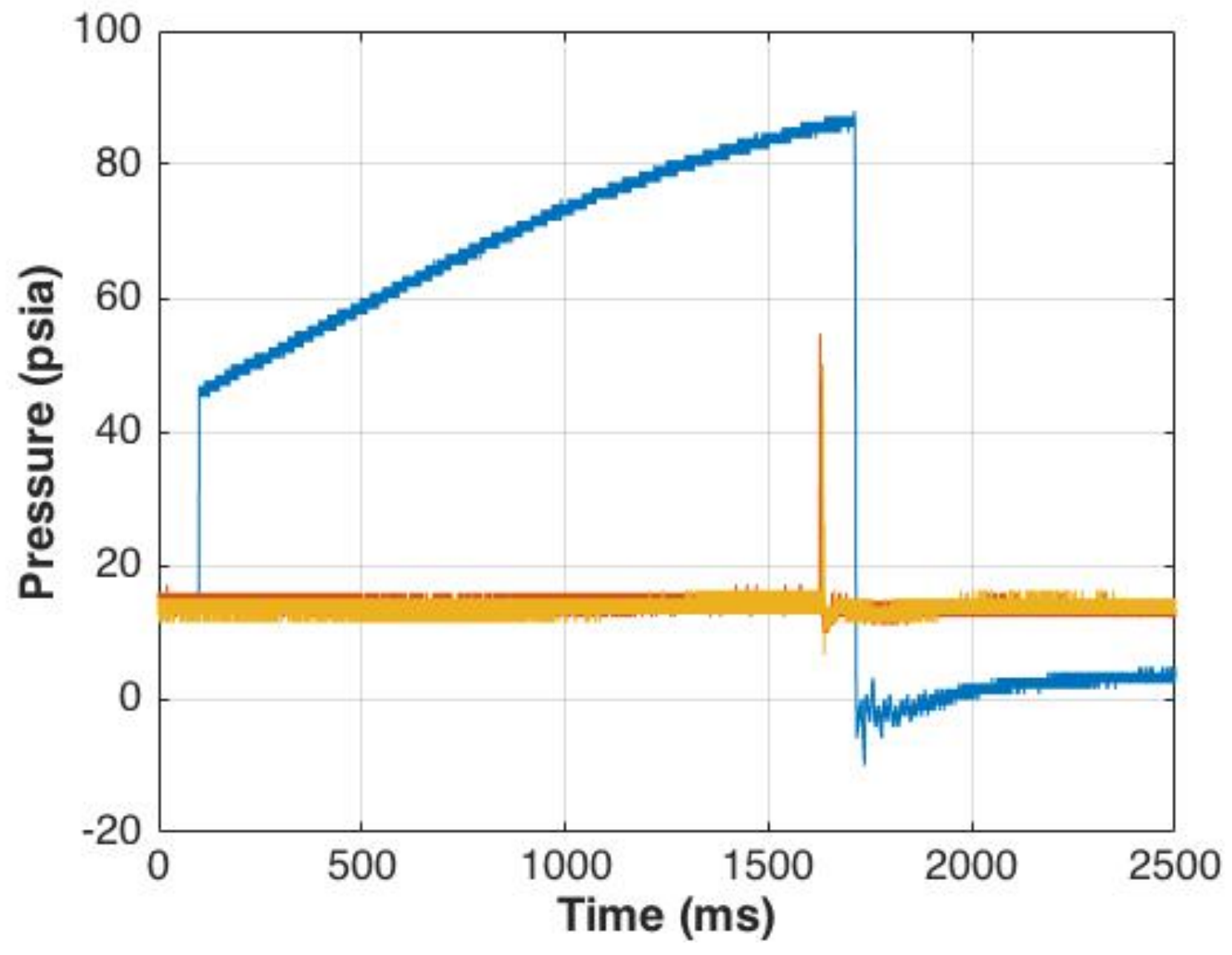

Figure D.2: Unfiltered AI Pressure Measurements 


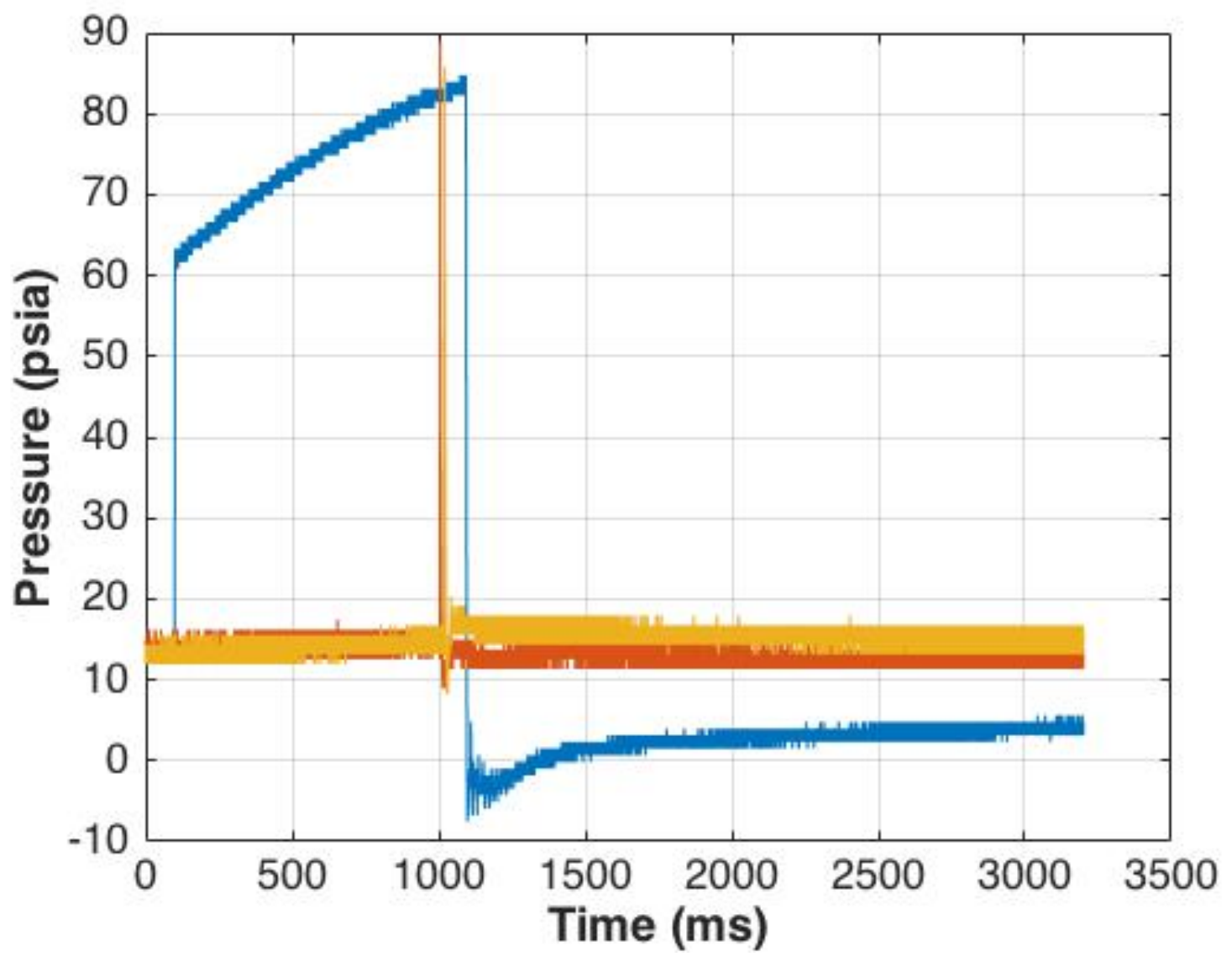

Figure D.3: Unfiltered AII Pressure Measurements 


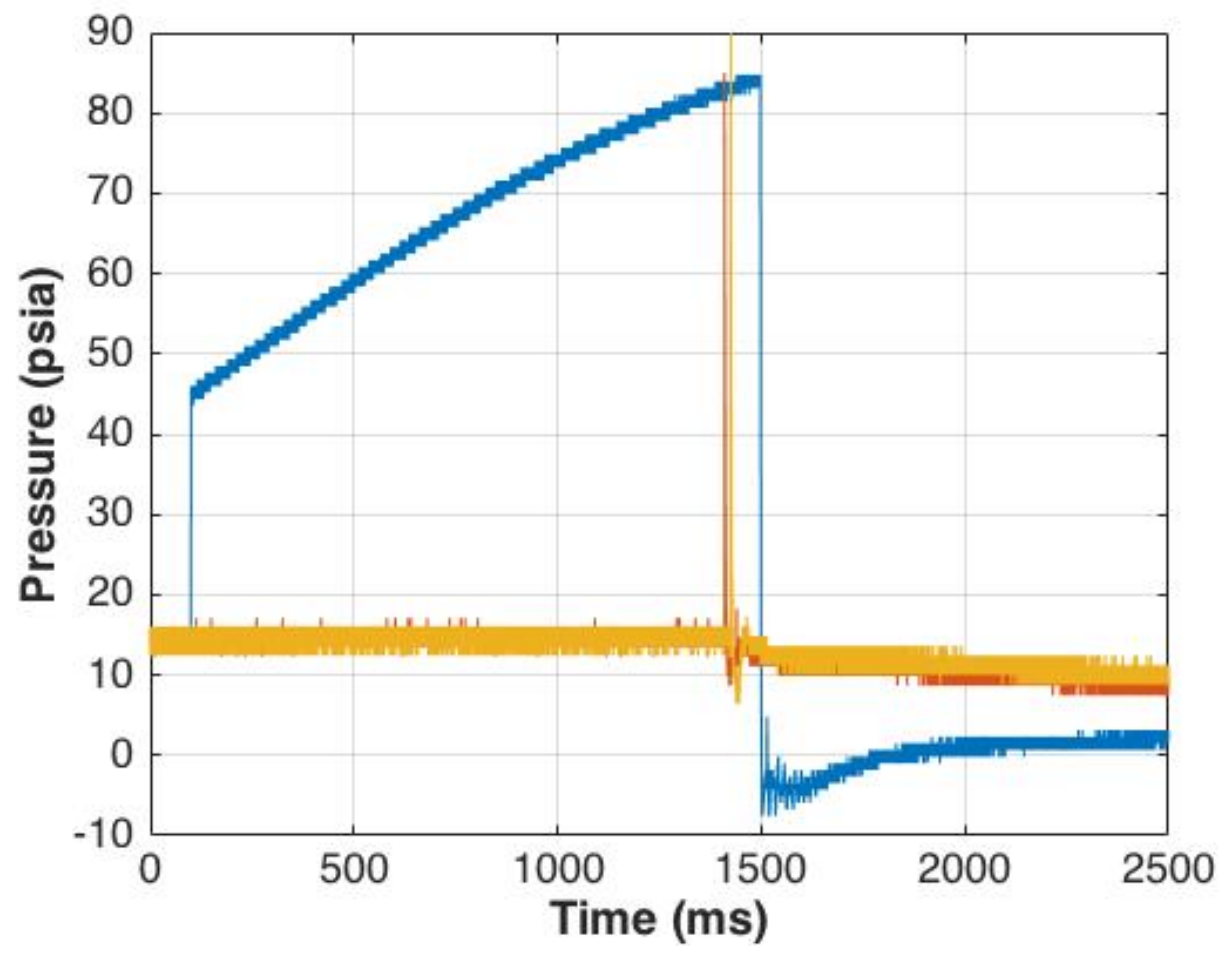

Figure D.4: Unfiltered BI Pressure Measurements 


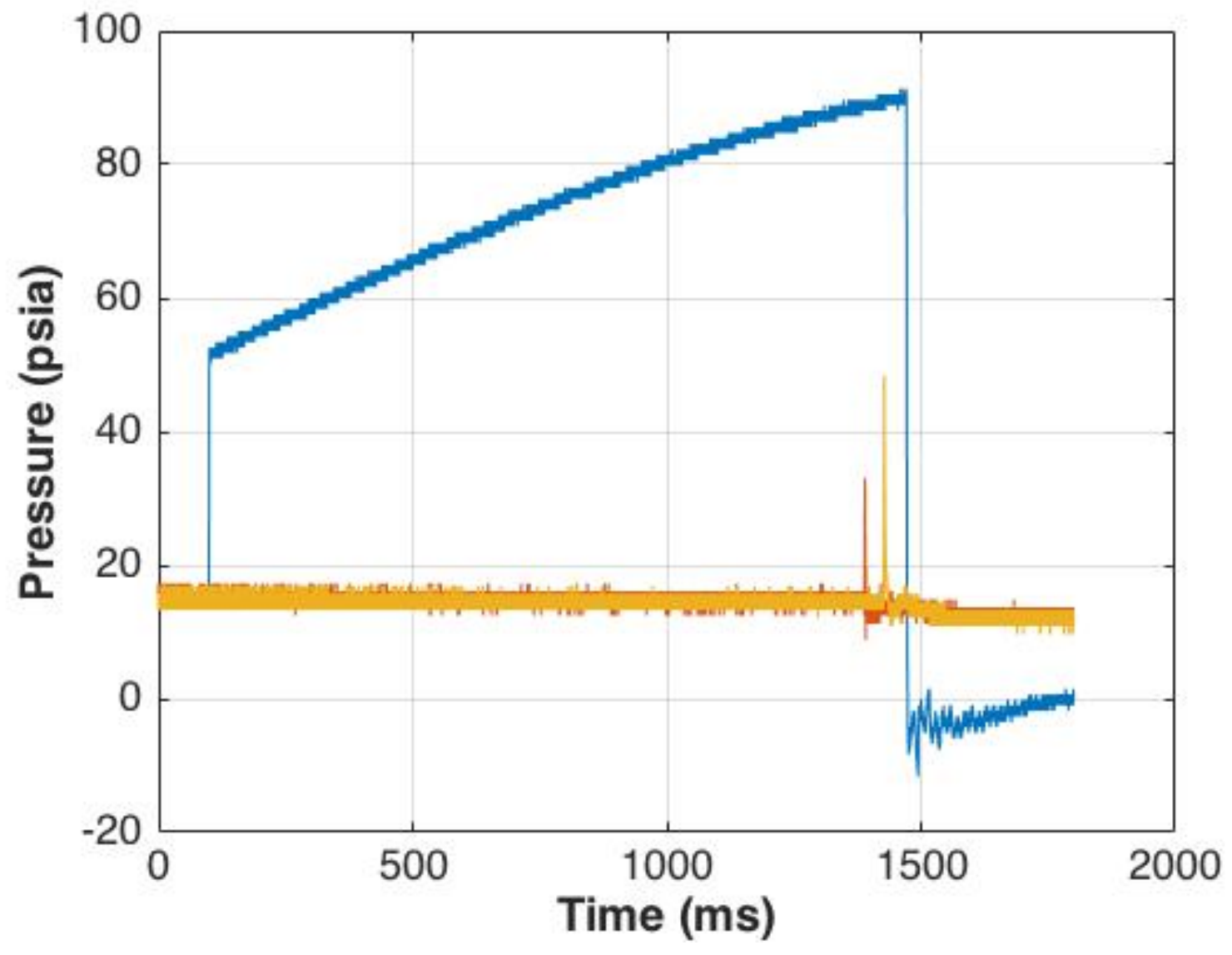

Figure D.5: Unfiltered BII Pressure Measurements 


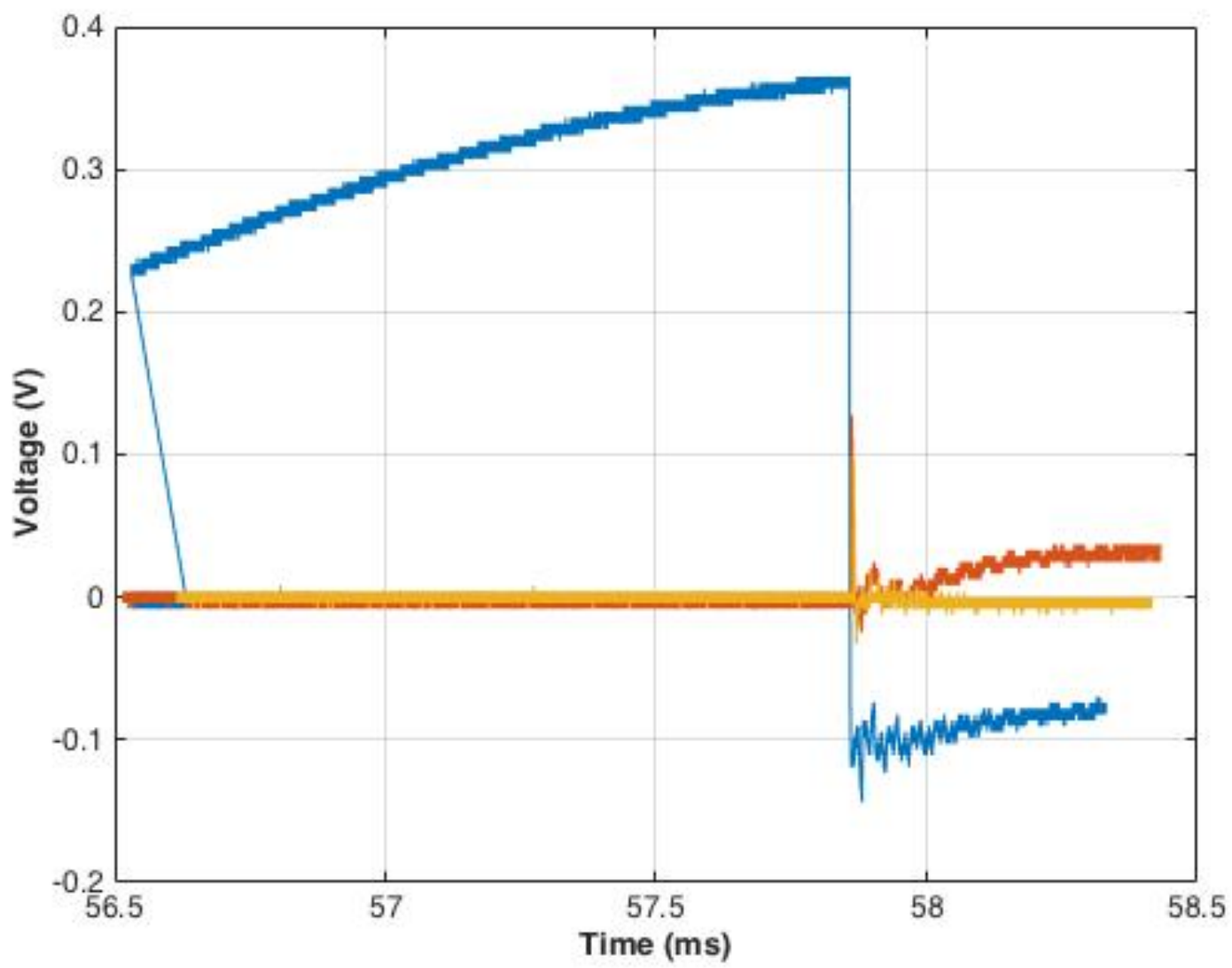

Figure D.6: Unfiltered Velocity/Pressure Measurements 